\title{
EXTRAVERSION AND COUNTER-DISPOSITIONAL BEHAVIOUR: EXPLORING CONSEQUENCES AND THE IMPACT OF SITUATION-BEHAVIOUR CONGRUENCE
}

\author{
by \\ Deanna C. Whelan

\begin{abstract}
A thesis submitted to
the Faculty of Graduate and Postdoctoral Affairs

in partial fulfillment of the requirements for the degree of

Doctor of Philosophy

in
\end{abstract} \\ Psychology \\ Carleton University \\ Ottawa, Canada
}

C2013 Deanna C Whelan 
EXTRAVERSION AND COUNTER-DISPOSITIONAL BEHAVIOUR

\section{Abstract}

People behave differently from one moment to the next and thus are frequently behaving 'out of character' or counter to their dispositions. Previous research exploring acting out of character indicated that acting extraverted was associated with increases in positive affect for both trait introverts and trait extraverts, whereas acting introverted was associated with cognitive control costs, but only for trait extraverts. These asymmetrical negative consequences can be partially explained by counter-dispositional behaviour as it may consume mental resources through monitoring and modifying behaviour. I proposed that previous research exploring counter-dispositional behaviour has neglected the importance of situational effects. Research demonstrates that contra-normative behaviour is depleting. Previous findings of costs for acting 'out of character' were limited to when behaviour was also incongruent with situational norms. Thus, I extended this line of inquiry by using the acting paradigm in introverted situations. I posited that there would be negative costs associated with acting extraverted, as it was contra-situational. I also posited that there would be even greater costs for dispositional introverts as they were also behaving counter-dispositionally. In two studies, I created introverted situations and randomly assigned participants to one of three experimental conditions (act extraverted, act introverted or control condition). In study $1(\mathrm{~N}=158)$, participants in the acting extraverted condition reported more positive affect, enjoyment, valence, and authenticity. Furthermore, no effects of cognitive fatigue were found. Thus, the hypothesized costs of acting extraverted were not found. In study $2(\mathrm{~N}=85)$ acting instructions were modified to highlight the positive features of introversion and the negative features of extraversion. Study 2 also employed naïve confederates so that feedback was uniform across 


\section{EXTRAVERSION AND COUNTER-DISPOSITIONAL BEHAVIOUR}

conditions. Unlike previous acting studies, study 2 found no benefits for acting

extraverted, but did find that participants reported the most effort and least authenticity

when acting extraverted. Study 2 results suggest contra-situational costs, but these did not differ by disposition. Previous research and study 1 demonstrate benefits for acting extraverted and suggest that introverts may be happier by behaving extraverted more often. Study 2 provides a critical caveat that this may not always be true. 


\section{EXTRAVERSION AND COUNTER-DISPOSITIONAL BEHAVIOUR}

\section{Acknowledgements}

The final version of this thesis does a great job of hiding the roadblocks, frustrations, and disappointments that were a part of its journey. It could not have been completed without all the support that I received from a great number of people. First, and foremost, I would like to thank John Zelenski who encouraged me to pick a topic that I was passionate about and not to be afraid of sticking my neck out in making my hypotheses. You are an excellent advisor, and I am happy to have been one of your students for a great number of years. You supported me throughout this project and especially when I needed it most. More importantly, you are also a friend, and I will always be grateful for that.

Thank you to my committee whose guidance and advice made this project better. I can look back at our earlier meetings with fondness and reassurance - at least I was not the only one who thought my hypotheses would turn out. How wrong we were!

Thank you to Brian Little. Our regular conversations fuelled my passion on this personal project and kept me thinking critically.

Thank you to my friends, family and CUHL mates. You put up with me talking about my thesis for many years. Providing input, suggestions, and comments about the project and on drafts - I greatly appreciated all of your perspectives and input. Thank you to Tom Foottit for all the support and encouragement you gave me while I was getting the document into a final draft form. And then for also willingly proof reading the entire document!

Finally, thank you to all the members of "Adventure Club" (both old and new). You made sure I kept active and involved in activities outside of my thesis. Dodgeball, 
EXTRAVERSION AND COUNTER-DISPOSITIONAL BEHAVIOUR

cake decorating, Spartan races, etc. all provided much needed distractions and also a

restorative niche for this crazy extravert. I like to think that these activities kept me sane

(or at least more sane that I would have been without you intervening in my life).

Three down.... zero to go. Does that mean I am actually done? 
EXTRAVERSION AND COUNTER-DISPOSITIONAL BEHAVIOUR

\section{Table of Contents}

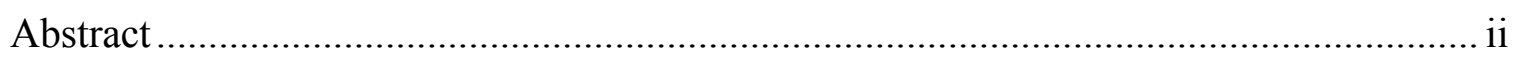

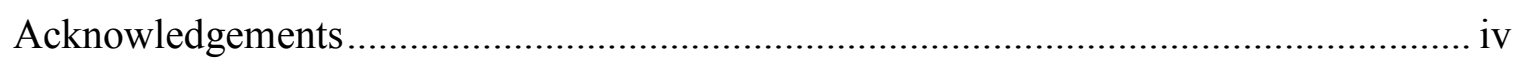

Table of Contents .................................................................................................... vi

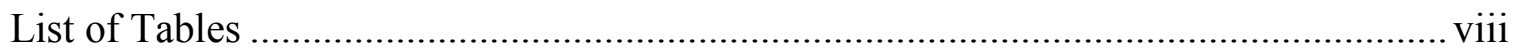

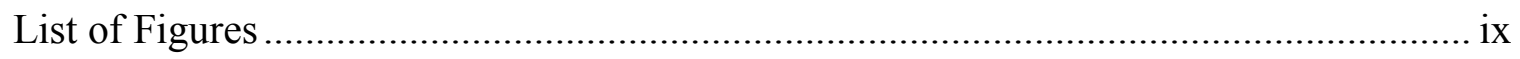

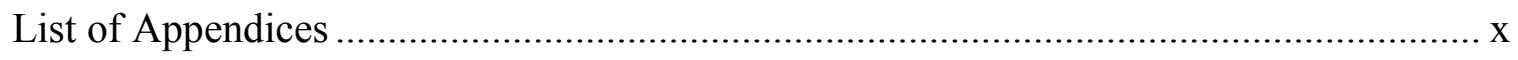

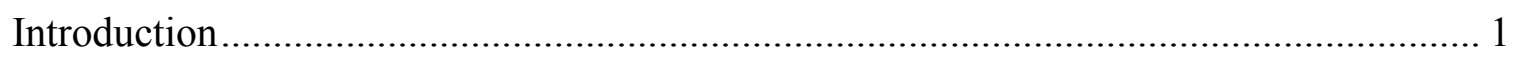

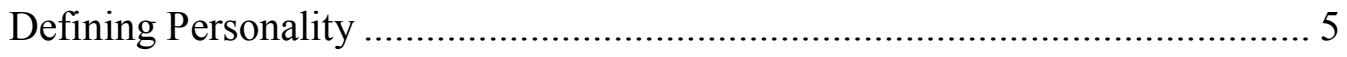

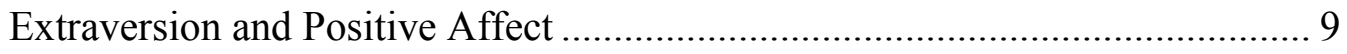

Theories Encompassing Variability in Behaviour ...................................... 10

Exploring Behaving Out of Character ....................................................... 14

Exploring Potential Costs for Acting Out of Character .............................. 17

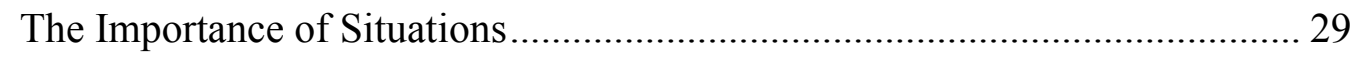

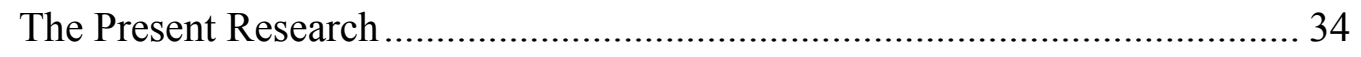

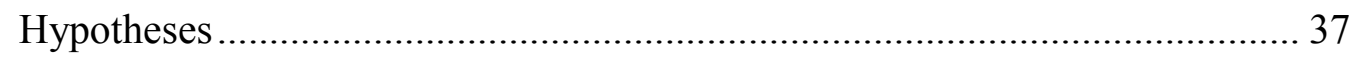

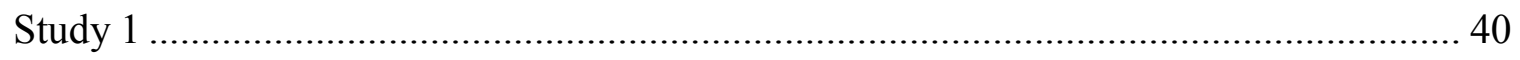

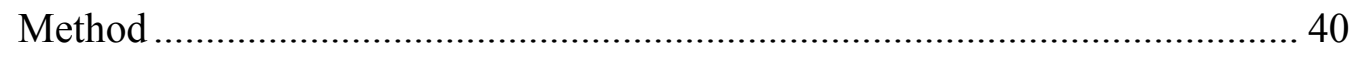

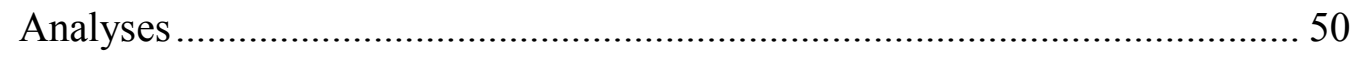

Preliminary Data Exploration ........................................................... 53 
EXTRAVERSION AND COUNTER-DISPOSITIONAL BEHAVIOUR

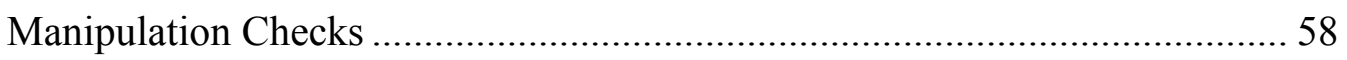

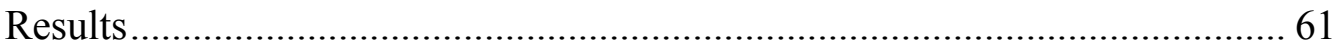

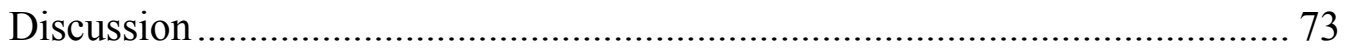

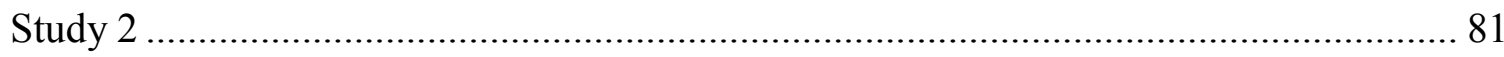

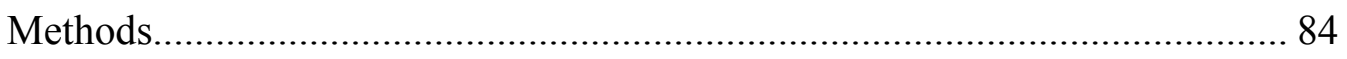

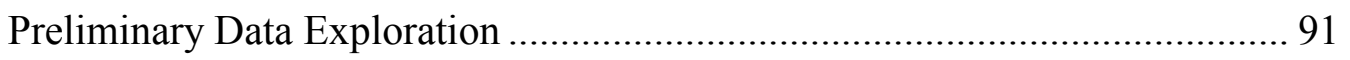

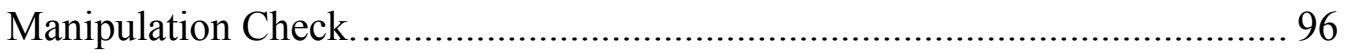

Assessment of Situation and Modified Instructions ................................... 98

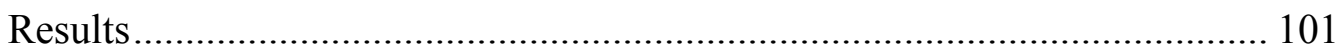

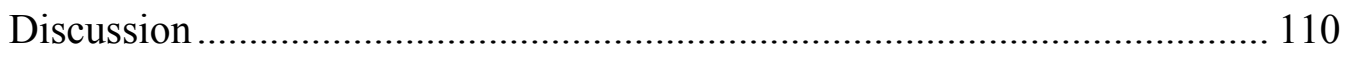

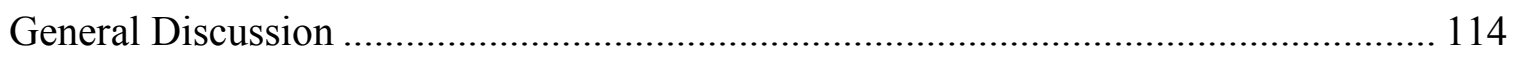

Strengths and Limitations ........................................................... 133

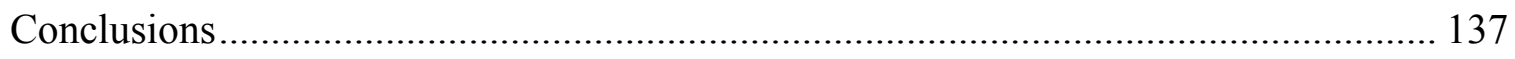

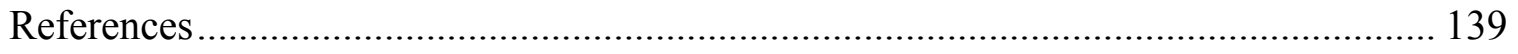




\section{EXTRAVERSION AND COUNTER-DISPOSITIONAL BEHAVIOUR}

\section{List of Tables}

Table 1 Correlations Between Self-Reported Behaviour and Affect for Study 1 ............. 55

Table 2 Correlations Between Observer-Rated Behaviour and Affect for Study 1 .......... 56

Table 3 Correlations Between Self-Reported and Observer-Rated Behaviour and Affect

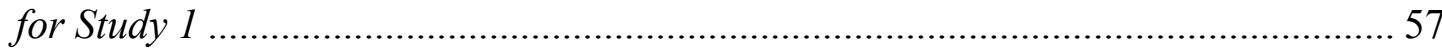

Table 4 Estimated Means by Experimental Condition Across all Dependent Variables for Study 1 72

Table 5 Multi-Parameter Tests and Hypothesis Testing Results for Condition Effect, Disposition Effect, and Their Interaction Across Cognitive Dependent Variables for Study 1 73

Table 6 Correlations Between Self-Reported Behaviour and Affect for Study 2 93

Table 7 Correlations Between Confederate-Rated Behaviour and Affect for Study 2 ..... 94 Table 8 Correlations Between Self-Reported Behaviour and Affect to Confederate-Rated Dependent Variables for Study 2 95

Table 9 Comparing Forecasted Affect by Condition and by Study 100

Table 10 Means by Condition for the Behaviour Items of Introverted and Extraverted 101 Table 11 Mean Differences by Condition For Each Dependent Variable for Study 2 ... 108 Table 12 Hierarchical Regression Results for Condition Effect, Disposition Effect and Their Interaction Across Dependent Variables Assessing Cognitive Fatigue for Study 2 109 


\section{EXTRAVERSION AND COUNTER-DISPOSITIONAL BEHAVIOUR}

\section{List of Figures}

Figure 1. The Affect Circumplex (Larsen \& Diener, 1992).......................................... 18

Figure 2. General pattern of trends of previous acting studies in the left panel, and the hypothesized trends for (unstudied) introverted situations in the right panel............ 38

Figure 3. Disposition by condition interaction on extraverted behaviour........................ 59

Figure 4. Interaction of condition by disposition for observer-rated negative affect...... 65

Figure 5. Interaction of condition by disposition for observer-rated self-consciousness. 66

Figure 6. Interaction of condition by disposition for arousal. ......................................... 67

Figure 7. Condition by disposition interaction for subjective authenticity..................... 69

Figure 8. Condition by disposition interaction for effort. ............................................. 70 
EXTRAVERSION AND COUNTER-DISPOSITIONAL BEHAVIOUR

\section{List of Appendices}

Appendix A: Personality Trait Questionnaire........................................................ 157

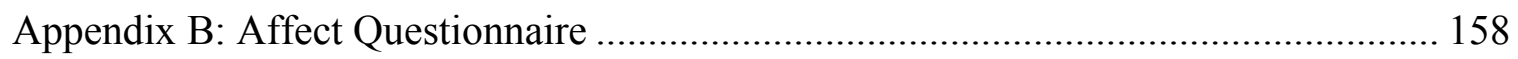

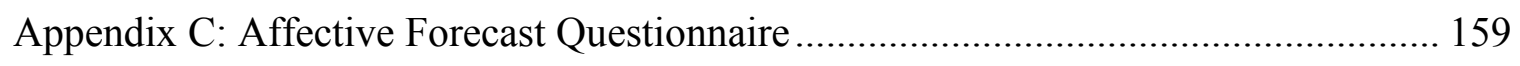

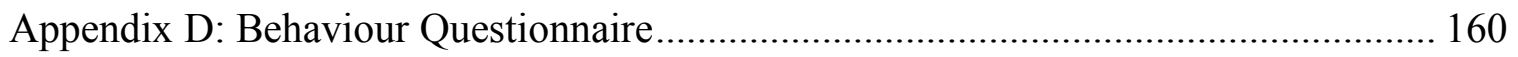

Appendix E: Authenticity and Effort Questionnaire .......................................... 161

Appendix F: Opinions About the Activity Questionnaire ...................................... 162

Appendix G: Rating of Other Participant Questionnaire - Study 1............................ 163

Appendix H: Instructions to Modify Behaviour Questionnaire - Study 1 ................... 164

Appendix I: Confederate Behaviour Rating Sheet - Study 2 ................................ 165

Appendix J: Confederate Affect Rating Sheet - Study 2 ...................................... 166

Appendix K: Instructions to Modify Behaviour Questionnaire - Study 2 ................... 167 
One of the most robust findings in the well-being literature is the link between extraversion and positive affect (Diener, Suh, Lucas, \& Smith, 1999). Despite numerous studies exploring this link, its primary cause is still debated. Yet the trend of extraverts, relative to introverts, reporting higher levels of positive affect is a consistent finding across a variety of methodologies (Diener et al., 1999; Lucas \& Fujita, 2000; Pavot, Diener, \& Fujita, 1990). A distinct approach to exploring a possible causal link between positive affect and extraversion was Fleeson, Malanos, and Achille's (2002) study that manipulated people's behaviour. They proposed that extraverted behaviour causes positive affect, and thus, having people behave in an extraverted way would result in positive affect. Although the premise appears self-evident, there is a counter intuitive element in that both introverts and extraverts would get the same increase in positive affect. Fleeson et al. (2002) had participants act extraverted or act introverted during group discussions. Their results supported the idea that extraverted behaviour causes positive affect. Surprisingly, the increases in positive affect for extraverted behaviour did not depend on disposition. That is, both introverts and extraverts reported higher levels of positive affect when acting extraverted relative to acting introverted.

Fleeson et al.'s (2002) results were somewhat surprising and counter intuitive in that counter-dispositional behaviour is theorized to have emotional costs (Côté \& Moskowitz, 1998; Little, 1996). Moreover, research on self-regulation suggests that monitoring and modifying behaviour is onerous and depletes a mental resource (Baumeister, 2002; Baumeister, Bratslavsky, Muraven, \& Tice, 1998). The mental 
resource is limited and when it is exhausted or depleted, termed ego depletion, there is a drop in performance on subsequent tasks requiring self-regulation and self-control. Therefore, it is possible that counter-dispositional behaviour is associated with benefits (i.e., the increased positive affect for acting extraverted) while also causing negative consequences (negative affect and ego depletion). For example, introverts may enjoy participating in a class discussion while also experiencing anxiety and mental exhaustion, and thus perform poorly on a test in the next class.

A collection of studies have explored ego depletion (Gallagher, Fleeson, \& Hoyle, 2011; Zelenski, Santoro, \& Whelan, 2012) and negative affect (McNiel \& Fleeson, 2006; McNiel, Lowman, \& Fleeson, 2009; Whelan \& Zelenski, 2007) resulting from acting out of character. These studies replicated Fleeson et al.'s original results of greater positive affect in the acting extraverted condition. No support for the idea of co-occurring negative affect when behaving counter-dispositionally was found. Importantly, costs in the form of ego depletion were found, but limited to extraverts in the acting introverted condition. That is, introverts in the acting extraverted condition reported an increase in positive affect with no associated impact on ego depletion or negative affect. These asymmetrical findings are further validated by a study that explored perceived effort for behaving extraverted and behaving introverted (Gallagher et al., 2011). They found that extraverts in the acting introverted condition reported greater effort than extraverts in the acting extraverted condition. There were no differences in perceived effort for dispositional introverts. That is, behaving introverted and behaving extraverted required similar levels of effort for trait introverts. Thus, across a series of studies and outcome measures, the general trend for acting out of character shows that the costs are largely 
limited to extraverts acting introverted. Greater positive affect and no negative implications are associated with the acting extraverted condition, including trait introverts in the acting extraverted condition. This leads to the question "why don't introverts act extraverted more?"

I postulate that past research exploring acting out of character is incomplete. Potential explanations for the asymmetrical findings have not been considered. Specifically, I argue that previous research on counter-dispositional behaviour has paid insufficient attention to situational factors. Research on ego depletion has established that behaving against situational norms (contra-normative ${ }^{1}$ behaviour) is depleting (Vohs, Baumeister, \& Ciarocco, 2005). Across all the acting studies, the situations have been moderately extraverted. Therefore, behaving contra-normatively is limited to the acting introverted condition, where negative consequences were found. Importantly, counterdispositional and contra-normative concepts each only partially explain the asymmetrical findings; they each predict things unsupported by the data unless the other is taken into account. That is, the concept of counter-dispositional behaviour also suggests costs for introverts in the acting extraverted condition, and the contra-normative concept also suggests costs for introverts (in addition to extraverts) in the acting introverted condition (assuming an extraverted situation). Results do not support these additional negative consequences. Importantly, in combination, the concepts of counter-dispositional and

${ }^{1}$ Behaving against the situational norm will be termed "contra-situational" whereas behaving against disposition will be termed "counter-dispositional." Admittedly, the contra and counter associations could be switched or be limited to one term for both ways of behaving in opposition. The distinction in terms is to assist in clarity and readability, particularly when interactions are discussed as both forms of oppositionalbehaviours have different trends associated with them. 
contra-normative behaviour may fully explain the asymmetrical pattern of results. That is, costs for situational self-regulation appear to be buffered by disposition.

The lack of diversity in the settings across the acting studies prevents uncoupling the contra-situational and counter-dispositional effects. To fill this gap, this research examines potential consequences for acting out of character in introverted settings. I hypothesized a mirror image of the previous asymmetrical findings. The contrasting combination of acting extraverted being contra-normative tested whether situational consequences of self-regulation are buffered by disposition. In two studies I examined the importance of situations for dispositionally-congruent and dispositionally-incongruent behaviours by employing novel situations.

Furthermore, I included a measure of authenticity to explore the novel premise that feeling authentic was dependent on situational norms. A related line of research has explored perceived authenticity, and established that more extraverted behaviour is associated with feeling more authenticity even for dispositional introverts (Fleeson \& Wilt, 2010). The experience sampling methodology of these studies indicates that these patterns are established across numerous settings. Of note, people normally behave similarly to situational norms (Pervin, 1968), and therefore, it is reasonable to assume that behaviours are congruent with the situation. I explored the novel position that subjective authenticity is dependent on situational norms. That is, behaviour needs to agree with the situational norms for it to feel authentic. Therefore, in contrast to Fleeson and Wilt's (2010) findings, I predicted that introverted behaviour would be associated with authenticity when it was congruent with situational norms. The experimental design of this research, combined with a measure of authenticity and manipulated behaviour, can 
demonstrate causal direction in a way that experience sampling methodology cannot.

Thus, I explored one possible causal direction between behaviour and authenticity, that is, if extraverted behaviour causes authenticity.

This research examined dispositionally congruent and incongruent behaviours in novel situations to explore the importance of situations. Similar to previous acting research, personality was measured and behaviour was manipulated. Across three conditions (acting extraverted, acting introverted, and control), I tested possible negative consequences (emotional, ego depletion, effort, and inauthenticity) for counterdispositional behaviour in introverted settings. Despite previous findings, I posited that there would be negative consequences for acting out of character (for both introverts and extraverts), but that they would be dependent on situational contexts. Therefore, because the situations were introverted, I posited that contra-normative, counter-dispositional behaviour (introverts behaving extraverted) would produce the greatest costs.

\section{Defining Personality}

Personality is an individual's characteristic pattern of thought, emotion, and behaviour. There is much information to consider in understanding an individual. Thus, most approaches to personality place restrictions or limitations on the kind or type of information that is considered or assessed. Variations in focus produce distinct approaches to the study of personality. For example, the trait approach measures individual differences whereas the learning or cognitive approach examines how people change their behaviour due to rewards and punishments. The phenomenological approach looks at people's conscious awareness and experiences whereas the biological approach examines biological mechanisms and evolution for how they are pertinent to 
personality. These approaches can be combined as each has unique advantages and disadvantages.

The concept of personality traits can be traced back to Aristotle (384 BC - 322 BC) with modern day theories having their origin with Sir Francis Galton (1884). Traits provide a way of comparing two or more individuals and thus focus on individual differences. As a result, traits disregard both characteristics that are common to all and characteristics that are truly unique. Two contentious questions in personality psychology are how many traits are there, and are traits the best unit for understanding personality (versus motivational needs, biological systems, etc.)? Murray conjectured that in order to understand a person, you must understand their needs and theorized twenty distinct needs while Block, adhering to a more Freudian perspective, theorized that only 2 characteristics were critical. Eysenck and Cattell used factor analysis to arrive at a number of traits but were differentially aided by biological considerations versus lists of trait words derived from the dictionary. Eysenck concluded three characteristics were needed whereas Cattell determined that sixteen characteristics were essential.

These different ways of classifying individuals' characteristics not only differ in the number of characteristics considered essential, but also differ on whether traits were conceptualized as how a person acted, were the sum of what an individual had learned, were based on biological structures, or were some combination of these concepts (see Matthews, Deary \& Whiteman, 2003 and Goldberg, 1993 for a general historical overview). Despite the diverse background of personality traits, there is emerging consensus by contemporary psychologists that most trait variation can be summarized by five broad, independent dimensions. One model in particular has come to the forefront 
and is termed the Big Five or the Five-Factor model (Digman, 1990; Goldberg, 1990).

One common set of labels for the five factors is extraversion, neuroticism, agreeableness, conscientiousness, and openness to experience. Theoretically these factors are distinct and orthogonal, although research frequently shows that the factors are associated (De Young, 2006; Digman, 1997). Furthermore, the factor labels conceal much complexity as each factor can be divided into narrower characteristics. Not surprisingly, there is a lack of agreement on the number of sub-factors and if they are termed facets or aspects. Earlier conceptualizations of personality traits can usually be described in terms of one, or more, of the Big Five traits. Thus, the Big Five is viewed as an integration of the other systems (Saucier \& Goldberg, 2003). Even without this integration, the large majority of conceptualizations of personality contain a dimension of extraversion (also labelled surgency) that is associated with the key features of sociability, assertiveness, activity, and excitement seeking. Its opposing term, introversion, has key features of being reserved, quiet, and having a preference for solitude (see Wilt \& Revelle, 2009, for a general review of extraversion and Watson \& Clark, 1997, for a review of theoretical stances of extraversion and their facets). The term shy is frequently incorporated into the concept of introversion, but an important distinction should be noted; not all introverts are shy. Both introverts and shy individuals have a preference for solitude but the individual labelled shy would be introverted but also score high on neuroticism (and particularly in social anxiety).

The two most prevalent methods for assessing extraversion, as well as other Big Five traits, are with trait-word adjectives (Goldberg, 1990) and sentence-length statements (Costa \& McCrae, 1989), both rated on Likert-type scales. Each factor is 
assessed as a dimensional score with people falling somewhere along the range of possible values and thus an individual can score high, low, or anywhere in between, for any particular trait. The labels represent polar extremes with some traits being known by both opposing terms (extraversion-introversion, neuroticism-emotional stability, etc.). Importantly, most people would not be completely extraverted or introverted, but would fall somewhere between these labelled extremes. In keeping with the Big Five measurement tradition, my methodology examines extraversion as a dimensional score, and the terms extravert or introvert will refer to people scoring higher or lower on the extraversion dimension without necessarily implying an extreme score.

One causal theory of introversion-extraversion is Gray's biopsychological theory of personality (Gray, 1970). Gray $(1972,1981)$ postulated two neurologically based systems termed the behavioural approach system (BAS) and the behavioural inhibition system (BIS). BAS was theorized to be sensitive to signals of reward and associated with approach motivation and positive emotions. BIS was theorized to be sensitive to signals of punishments and associated with avoidance motivation and negative emotions (Carver \& White, 1994). An extraverted individual is posited to have a more active BAS, whereas a neurotic individual is posited to have a more active BIS (Rusting \& Larsen, 1997; Fischer, Wik, \& Fredrikson, 1997).

In this framework, a neurotic-introvert would be receiving primarily stop signals or avoidance motivation. Conversely, stable-extraverts would be receiving primarily go signals or approach motivation. Of course, these systems can be in conflict with the neurotic-extravert simultaneously receiving signals of go and stop. Approach motivation has been advanced as a key feature of extraversion and posited to underlie extraversion's 
link to positive affect (Carver, Sutton, \& Scheier, 2000).

\section{Extraversion and Positive Affect}

The relationship between extraversion and positive affect is one of the most robust and consistently replicated findings in the subjective well-being literature (Pavot et al., 1990). Research has clearly established that extraversion is associated with positive affect while neuroticism is associated with negative affect. The relationship between extraversion and positive affect is such that, on average, extraverts report more positive affect relative to introverts (Diener \& Seligman, 2002; Hills \& Argyle, 2001; Lucas \& Baird, 2004; Lucas \& Fujita, 2000). The link between extraversion and greater positive emotions does not indicate that introverts are unhappy or depressed per se, but simply that extraverts are generally happier than introverts (see Zelenski, Sobocko, \& Whelan, 2014, for a review of this issue). The relationship between neuroticism and negative affect is such that, on average, highly neurotic people report more negative affect relative to emotionally stable individuals (Costa \& McCrae, 1980; Charles, Reynolds, \& Gatz, 2001; McCrae \& Costa, 1991; Rusting \& Larsen, 1997). As noted above, neuroticism is independent (orthogonal) from extraversion meaning that an extravert is equally likely to score high or low on neuroticism. The most beneficial combination of these two traits (for happiness) would be emotionally stable-extraversion whereas the worst combination would be neurotic-introversion.

Most research on extraversion and positive affect focuses on the various ways these aspects could be linked. The greater BAS and approach motivation of extraverts suggests that extraverts react more strongly to positive stimuli (Larsen \& Ketelaar, 1991; Zelenski \& Larsen, 1999), and extraverts may select/approach situations that are more 
conducive to positive emotions (i.e., social situations, Pavot et al., 1990). Other suggested links for extraversion-positive affect association include that extraverts may also be better able to adapt to frequent social interactions (i.e., more social skills, Emmons, Diener, \& Larsen, 1985), or they may simply have a higher 'default' level of positive mood (Lucas \& Baird, 2004), or are better able to maintain positive moods when they do occur (Lischetzke \& Eid, 2006). The numerous studies demonstrating different explanations for the link between extraversion and positive affect exemplifies the robustness of the association.

Fleeson et al. (2002) proposed that extraversion and positive emotions are associated because extraverts behave extraverted more frequently. To appreciate this explanation requires acknowledging that people's behaviour is not entirely consistent. A number of personality theories explore the notion that people behave differently from one moment to the next. The density distribution of states, the behaviour concordance model, and free trait theory all acknowledge variability in behaviour.

\section{Theories Encompassing Variability in Behaviour}

The density distribution of states theory (Fleeson, 2001, 2004) proposes that personality traits are the accumulation of everyday behaviour. Therefore, what a person actually does defines who they are in terms of their personality. That is, we know that someone is extraverted because they frequently behave extraverted. Thus, the density distribution emphasizes behaviour as a core component of personality, such that, 'having' a personality indicates that the behavioural aspect of the trait is enacted frequently. A key premise behind Fleeson's density distribution approach is that personality states are largely isomorphic with personality traits. That is, states have the same content and 
breadth (i.e., behaviours, thoughts, and emotions) as traits (Fleeson \& Leicht, 2006) but differ in that states are "in the moment" whereas traits refer to averages over more prolonged periods.

Fleeson $(2001,2004)$ measured state behaviours, employing experience sampling methodology, with adjectives indicative of behaviour (e.g., talkative, bold). These adjectives were taken from measures of traits such as Goldberg's Markers (Goldberg, 1992). He showed that most people expressed a wide range of behaviour for most traits. Furthermore, behaviour shifted across the range in relatively short time frames. Key is that the central tendency (mean or median) differs from person to person. That is, trait introverts and extraverts differ in their average level of extraverted behaviour (aggregated over time), although both can behave introverted or extraverted at any particular moment. In fact, Fleeson (2001) demonstrated that a person's behaviour differs more from moment to moment (within-person variability) than two people differ from each other (betweenperson variability). A pivotal component of the density distribution theory, in relation to the proposed research, is that behaviour variability can be due to external and internal influences such as projects and goals (Little, 1983), situational norms (Reis \& Collins, 2004), and emotional states (Cunningham 1988; Whelan \& Zelenski, 2012a).

Similar to the density distribution model, the self-concordance model (Côté \& Moskowitz, 1998; Moskowitz \& Côté, 1995) focuses on variation in behaviour but differs in some predictions. It postulates that pleasant affect results when behaviours align with disposition (i.e., concordance between behaviour and trait). The self-concordance model explores behaviour and traits using the interpersonal circumplex, a model that organizes interpersonal traits with independent dimensions. One axis is anchored by 
assertive/dominant versus passive/submissive. The second axis is anchored by warm/agreeable versus hostile/quarrelsome. Results have generally supported the behavioural concordance model for agreeable/disagreeable behaviours in that agreeable individuals report more pleasant affect for behaving agreeably and more unpleasant affect when behaving disagreeably, compared to disagreeable individuals. That is, agreeable individuals enjoy behaving agreeably. Although Côté and Moskowitz (1998; Moskowitz \& Côté, 1995) note that the majority of their sample demonstrate the trend of pleasant emotions for agreeable behaviour, they also found that disagreeable individuals report more pleasant affect when behaving disagreeably and more unpleasant affect when behaving agreeable (e.g., Oscar the Grouch is happier being a grouch than being amicable!).

The behaviour concordance model, in agreement with the density distribution model, would predict extraverts reporting positive emotions for behaving extraverted. The behavioural concordance model predicts that introverts would feel positive emotions when behaving in accordance with their personality (i.e., introverted). This contrasts with the density distribution model that predicts introverts experiencing positive affect when behaving extraverted. Studies support the idea that introverts report more positive affect when acting extraverted compared to when acting introverted (Fleeson et al., 2002; Zelenski et al., 2012) and thus results support the density distribution model.

Free trait theory (Little, 1996, 2000), similar to self-concordance and density distribution models, focuses on variation in behaviour. It diverges from the density distribution of states model by proposing costs to emotional and physical well-being for behaving counter to dispositions. Importantly, free trait theory proposes that the costs 
manifest only after prolonged periods of acting against one's natures and thus differs from the behavioural concordance model. Little $(1996,2000,2008)$ proposes three natures that each influence behaviour and proposes that it is the combination of them, and their interactions, that result in variation in behaviour.

The "first nature" of the free trait theory is biological and influences behaviour through neurophysiological mechanisms. The "second nature" is social and influences behaviour through social norms and expectations. The "third nature" is personalized and influences through motivation such as goals (Little \& Joseph, 2007; McCabe \& Fleeson, 2012). The components are each independent and as such, can align in their influence or conflict. For example, a biologically extraverted individual at a funeral, with a personal objective to remain quiet and not make a scene, will probably remain subdued and respectful. In this example, the social context and personal motivation align but are in conflict with the biological disposition of the individual. The combination and interaction of the three natures are posited to determine behaviour and thus, there is variability in behaviour as a function of the influences of the three natures.

The free trait theory is augmented by three additional assumptions that extend the theory to consequences of behaviour. Specifically, acting out of character for long periods of time is posited to exact repercussions in the form of costs to physical and emotional well-being. There is a caveat that "restorative niches," environments in which individuals can be their biological selves, can mitigate the costs. The third assumption is that environments differ in the extent to which they afford the opportunity to restore first natures. Therefore, the behavioural concordance model and free trait theory both propose that behaviour is variable and behaving counter-dispositionally is associated with 
unpleasant emotions, but the free trait theory also requires that the out of character behaviour is enacted for a prolonged period and that niches can alleviate the effects. These two models contrast with the density distribution model in that behaviour is recognized as variable but there are no costs proposed in the model.

\section{Exploring Behaving Out of Character}

Fleeson et al. (2002) examined short-term within-person variations in extraverted behaviour to determine if the link between extraversion and positive affect found in aggregate between-person research also occurred within-person. As previously noted, the majority of people express a full range of extraverted behaviour across relatively short periods of time (Fleeson, 2001). Furthermore, Fleeson and colleagues proposed that the correlation between extraverted behaviour and positive affect found in aggregate form would hold at the idiographic level. This leads to the counter-intuitive hypothesis that introverts would experience positive affect when behaving extraverted (i.e., counterdispositionally). Fleeson et al. (2002) tested this in two ways, by examining the momentto-moment correlations with experience sampling methodology (ESM), and manipulating extraverted behaviour in a laboratory setting. Through manipulating state extraversion, instructing participants to act extraverted (bold, assertive, and adventurous) or introverted (quiet, shy, and unadventurous) during a group discussion task, and reporting subsequent positive affect, they could explore whether or not extraverted behaviour caused positive affect. Across methodologies, they found higher levels of momentary extraverted behaviour associated with greater positive affect compared to introverted behaviour. Importantly, this relationship held for both trait introverts and extraverts (Fleeson et al., 2002). In sum, the link between extraversion and positive affect held at the within-person 
level.

Fleeson and colleagues' finding that extraverted behaviour causes positive affect for both dispositions was novel, and somewhat surprising. Based on these findings, they suggested that acting extraverted had possible value as a clinical intervention to enhance well-being. That is, increasing extraverted behaviour could be used to increase positive affect and thus inform clinical interventions on mental health (Fleeson et al., 2002; McNiel \& Fleeson, 2006). Unfortunately, there were some methodological issues that provided reasons to question the generalizability of their findings. Subsequent studies (Gallagher et al., 2011; McNiel \& Fleeson, 2006; Whelan \& Zelenski, 2007; Zelenski et al., 2012, 2013 ${ }^{2}$ ) have replicated Fleeson et al.’s (2002) acting extraverted methodology while addressing confounds and elaborating on possible implications of acting counterdispositionally.

Fleeson et al.'s (2002) acting study had participants reflect on a time where they behaved similar to the described behaviour listed in the instructions. This reflection activity may have resulted in thinking of more pleasant instances in the acting extraverted condition. "Talkative, bold and assertive" behaviours would occur in more social settings compared to "shy, quiet, and unadventurous" behaviours and social situations are generally more pleasant than non-social situations (Lucas \& Diener, 2001; Whelan \& Zelenski, 2012). Therefore, the reflection exercise may have unintentionally caused the differences in positive affect found between conditions. All subsequent studies have eliminated the reflection exercise and still reproduced positive affect differences between

${ }^{2}$ Study 5 in the Zelenski et al.(2013) paper is the dataset presented as study 1 in this document. 
conditions. Subsequent studies have also employed observer ratings, either by blind (to instructions) confederates (Gillen, 2009; Zelenski et al., 2012) or participants in a control condition where there are no instructions to modify behaviour (McNiel \& Fleeson, 2006; Whelan \& Zelenski, 2007; Zelenski et al., 2012), and results confirm that participants follow instructions to alter their behaviour. The addition of a control condition has also clarified the interpretation of results to determine if acting extraverted increased positive affect, or the opposite, that acting introverted decreased positive affect. Zelenski and colleagues found that participants in the acting extraverted condition reported more positive affect compared to those in the acting introverted and control conditions (Gillen, 2009; Whelan \& Zelenski, 2007; Zelenski et al., 2012 ${ }^{3}$ ). Thus, their results support the notion that acting extraverted increases positive emotions. Subsequent studies have extended the acting out of character methodology to longer group discussion times (Zelenski et al, 2012), to the full range of trait extraversion as opposed to sampling from extreme groups (Whelan \& Zelenski, 2007; Zelenski et al., 2012), and extending to dyadic interactions for both a game setting (Gillen, 2009) and an interview setting (Zelenski et al., 2012). In combination, the collection of acting studies have replicated the pattern of findings of Fleeson et al. (2002) and extended the interpretation and generalizability while addressing possible confounds.

${ }^{3}$ Some studies show no significant difference between acting extraverted and control conditions although the direction of the difference was in the correct direction. Study one had a significant difference between acting extraverted and control condition with acting extraverted reporting more positive affect. In study two, acting extraverted and control conditions did not differ in positive affect although the trend of more positive affect reported for the acting extraverted condition held. Of note is that even when all conditions do differ, the control and acting extraverted conditions are more similar than the control and acting introverted conditions. 
Experience Sampling methodology has also explored if state extraverted behaviour can mediate the trait extraversion-trait positive affect association. Thus, they considered that one possible reason for trait extraverts' greater happiness was their more frequent extraverted behaviour. Wilt, Noftle, Fleeson, and Spain (2012) found that more frequent enactment of extraverted behaviour resulted in more frequent state positive affect, which in turn was associated with greater trait positive affect. Consequently, the positive benefits of behaving extraverted have been found in lab environment and natural settings, and extended to one reason for why extraverts are happier. Even with all acting studies finding that acting extraverted appears beneficial as it causes positive affect; finding benefits for acting extraverted does not exclude costs for enacting the behaviour.

\section{Exploring Potential Costs for Acting Out of Character}

Individuals vary their level of extraverted behaviour such that the full range of extraverted behaviours are expressed over relatively short periods of time (Fleeson, 2001). Consequently, even dispositional introverts regularly and routinely behave extraverted. If acting extraverted feels good (increases positive affect) why don't introverts do it more? One possible explanation behind introverts behaving dispositionally-congruent, despite the increases to positive affect for counter-dispositional behaviour, is that there are simultaneous costs for counter-dispositional behaviour. For example, there may be a corresponding increase to negative affect for acting out of character. In addition, monitoring and modifying behaviour may feel inauthentic, require greater effort or result in cognitive fatigue.

Affect circumplex. Affect is frequently conceptualized as being composed of two dimensions: valence and arousal (Larsen \& Diener, 1992). These dimensions are 
independent as an emotional experience can be high or low on arousal and

simultaneously pleasant or unpleasant. The affect circumplex is a two-dimensional

structure that represents this conceptualization (see Figure 1). Valence forms the

horizontal axis and is anchored by terms such as pleasant and unpleasant. Arousal forms

the vertical axis and is anchored by terms such as active and inactive (Russell, 1980).

The spacing of terms is such that items at 180 degrees are bipolar whereas items in closer proximity are correlated with their closeness indicating the strength of the correlation.

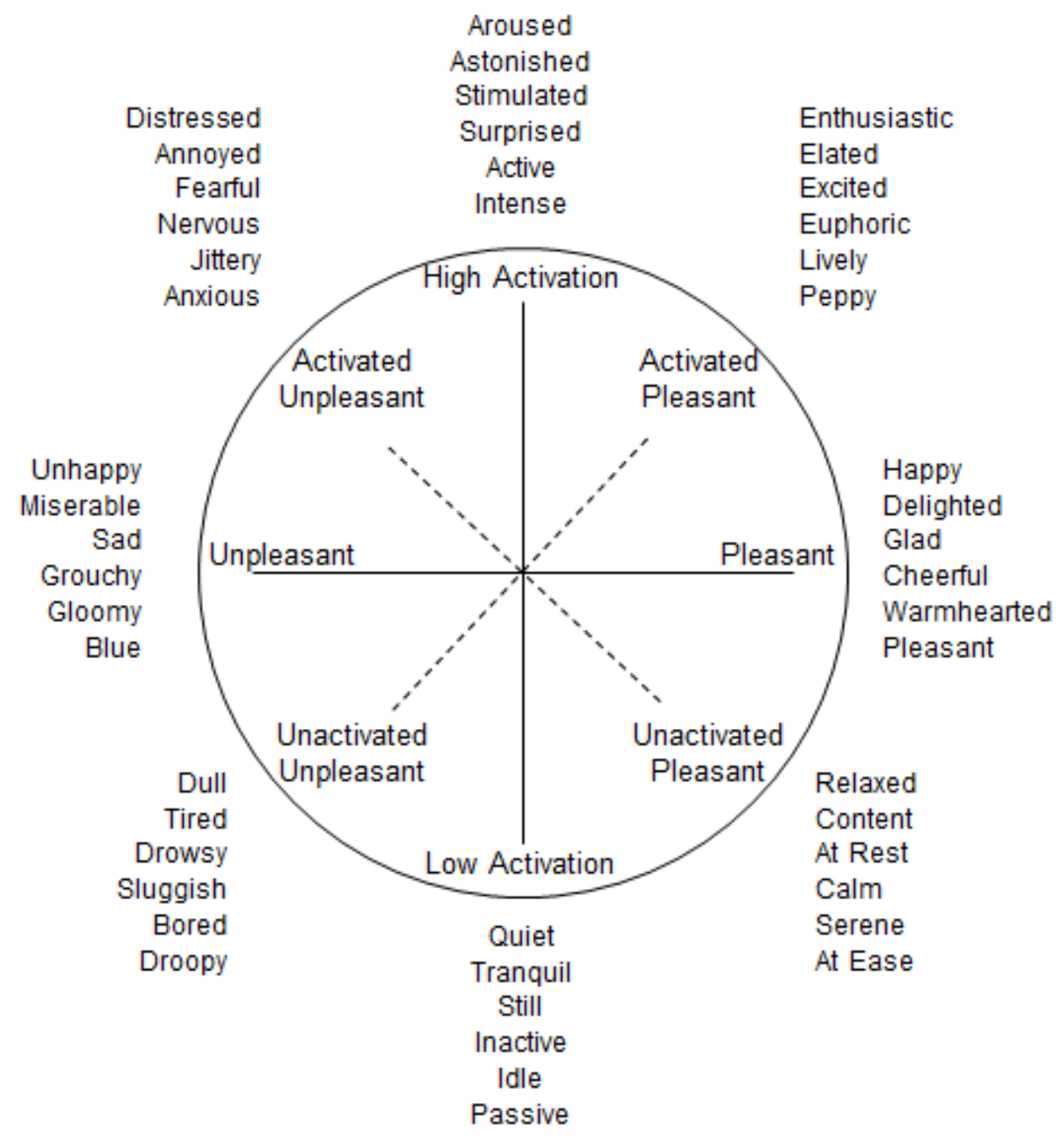

Figure 1. The Affect Circumplex (Larsen \& Diener, 1992).

Positive affect is defined as high arousal pleasant emotions. Negative affect is 
defined as high arousal unpleasant emotions. Note that both positive affect and negative affect are high arousal affective experiences. In this way, a low score on positive affect (excited) does not indicate low arousal (relaxed) but simply a lack of high arousal. Interestingly, positive affect and negative affect are orthogonal factors (Russell \& Carroll, 1999; Watson, Clark, \& Tellegen, 1988). That is, an increase in one does not equate to a decrease in the other. Furthermore, positive affect and negative affect can co-occur, as indicated by their spacing on the affect circumplex (see Warr, Barter, \& Brownbridge, 1983 for an exploration of the independence of positive and negative affect). Additionally, research demonstrates that positive and negative affect can be simultaneously experienced, at moderate levels, in experimental and real world environments (Diener, Larsen, Levine, \& Emmons, 1985; Larsen, McGraw, \& Cacioppo, 2001; Zelenski \& Larsen, 2000).

Negative Affect. As positive affect is orthogonal to negative affect, a plausible reason for why introverts do not behave extraverted is a corresponding increase in negative affect. That is, in acting studies the introverts in the acting extraverted condition may have been experiencing positive affect (excited) while simultaneously experiencing negative affect (anxious). To address this possibility, studies assessed negative affect following the group discussions (McNiel \& Fleeson, 2006; Whelan \& Zelenski, 2007; Zelenski et al., 2012). Across studies, self-reported negative affect was low with no differences between conditions or by disposition. Furthermore, there was no support for a condition by disposition interaction on negative affect. Studies exploring negative affect have found no support for it as a cost for acting out of character in this domain (McNiel \& Fleeson, 2006; Whelan \& Zelenski, 2007; Zelenski et al., 2012). Therefore, 
the question remains as to why introverts do not behave more extraverted.

The broad concept of negative affect has been explored as a potentially cooccurring consequence to acting extraverted with no support found thus far. More specific forms of unpleasant affects have not been examined. One potentially promising avenue is the concept of self-consciousness. Perhaps introverts do not act extraverted in order to avoid feeling self-conscious.

Emotional valence. Another possible explanation for why introverts do not behave extraverted more frequently, and why there has not been a corresponding increase to negative affect following acting extraverted, is that the acting instructions manipulated arousal and not valence per se. If acting extraverted is more arousing than acting introverted, the greater positive affect reported by Fleeson et al may have been due to an increase in arousal and not specifically a pleasant valence. Similarly, introverted participants acting extraverted may have been experiencing unpleasant affect but not a high arousal unpleasant affect (negative affect). To test the affective implications of acting extraverted, subsequent studies have measured emotions more comprehensively including separate analyses for arousal and emotional valence (McNiel et al, 2010; Whelan \& Zelenski, 2007). They replicated the trend of greater positive affect for the acting extraverted condition, versus acting introverted in group discussions (Whelan \& Zelenski, 2007) and dyadic interactions (McNiel et al., 2010). Participants also reported greater pleasant valence and arousal when acting extraverted versus acting introverted. Therefore, acting extraverted is associated with more arousing and more positive emotions. Thus, the possible explanation of acting instruction manipulating only arousal, and not valence, is discounted. 
Authenticity. Despite the numerous researchers who use the term authenticity, there is no agreed upon definition. Maslow (1968) conceptualizes authenticity as a discovery of one's true inner nature; Rogers (1961) notes that it is a congruence between one's self-concept and life experiences. Deci and Ryan $(1995,2000)$ postulate that authenticity is a bi-product of self-regulation that reflects high levels of selfdetermination (versus externally mandated behaviour). Kernis and Goldman (2005) define authenticity as behaving in a way that is in agreement with one's values and preferences - termed one's true or core self. Despite the varied definitions and conceptualizations of authentic self, even adolescents can distinguish between behaving in a way that is true to themselves versus showing a false-self (Harter, 2002). Behaving authentically or in accordance with one's character is valued within society (Harter, 2002; Ryan \& Deci, 2004). Consistent with the value placed on authenticity, research confirms that self-reported authenticity is linked with positive life outcomes, including positive affect, self-esteem (Schlegel, Hicks, Arndt, \& King, 2009), life satisfaction, and lower negative affect (Kernis \& Goldman, 2005).

In relation to the proposed research, Fleeson and Wilt (2010) explored disposition-behaviour alignment and its association with authenticity. That is, they explored whether or not people felt more authentic when behaving in a manner that corresponded to their disposition. Fleeson reasoned that people would see authenticity as stemming from behaving consistent with dispositions. To determine if this is a more generally held belief, Fleeson and Wilt (2010) had participants complete two personality measures; a standard personality assessment and one with modified instructions asking participants to indicate how they behave when being their true self as a proxy for 
authentic behaviour. Fleeson and Wilt then correlated the two measures and found that participants reported feeling most authentic when retrospective behaviour aligned with personality. That is, introverts reported feeling most authentic when their behaviour was introverted and extraverts reported feeling most authentic when their behaviour was extraverted. Importantly, this study only examined retrospective behaviour, which may be subject to bias. Fleeson and Wilt expanded this line of inquiry by assessing authenticity and behaviour in the moment.

In three studies, Fleeson and Wilt (2010) examined trait-consistency (the alignment between personality trait and state behaviour) and authenticity. The traitconsistency hypothesis acknowledges that people's behaviour varies from moment to moment and focuses on how the variation in personality states fluctuates with authenticity. They examined the within-person variability of state behaviour and authenticity employing both repeated measures and experience sampling methodology in exploration of the trait-consistency hypothesis.

Across three studies, results did not support trait-consistency as state-trait discrepancy did not predict authenticity. Interestingly, they found positive correlations for behaviour and authenticity such that behaviour that was more extraverted, agreeable, conscientious, emotionally stable and open was perceived as more authentic. Furthermore, this trend did not differ by disposition. That is, both introverts and extraverts reported greater subjective authenticity when behaving extraverted. Fleeson and Wilt concluded that people accept variability in behaviour as a component of their self-concept and that flexibility in behaviour can be genuine. However, as they did not manipulate behaviour, the causal direction was not established. That is, behaving 
extraverted may lead to feeling authentic or feeling authentic may lead to behaving extraverted.

Importantly, similar to the counter-dispositional asymmetric findings from the acting extraverted studies, where introverts report greater positive affect when acting extraverted, Fleeson and Wilt (2010) found that introverts report feeling more authentic when behaving extraverted. This contradicts people's expectations as the retrospective reports show that introverts reported introverted behaviour as authentic. The proposed research included a measure of subjective authenticity as a potential cost for manipulated behaviour.

Effort. Gallagher et al. (2011) have explored effort in acting out of character. They had participants act extraverted or act introverted using a modified version of the group discussion methodology of Fleeson et al. (2002). Gallagher et al. (2011) argued one possible explanation for disposition's stability is that counter-dispositional behaviour is effortful. Trait behaviour was defined as typical or habitual ways of behaving and divergence from typical was assumed to require effort. Thus, in agreement with selfconcordance and free trait theories, they hypothesized that acting counter-dispositionally would be associated with greater effort compared to acting dispositionally-congruent. That is, introverts behaving introverted would require less effort, as this is their peak frequency or habitual way of behaving, relative to introverts behaving extraverted. Results partially support their hypothesis in that extraverts in the acting introverted condition (i.e., counter-dispositional) reported more effort relative to extraverts in a dispositionally-congruent condition. However, the hypothesis was not supported by introverts acting extraverted; introverts did not differ in reported effort when acting 
dispositionally-congruent or counter-dispositionally. These asymmetrical results therefore add an element of support for acting extraverted as a method for increasing well-being while adding to the perplexity about why introverts do not behave extraverted more often.

Ego depletion. A similar line of inquiry to that of Gallagher et al.'s (2010) exploration of effort is that of self-regulation. Self-control resources are depleted through monitoring and modifying (regulating) behaviour. The resulting depleted state is termed ego depletion. Zelenski et al. (2012) proposed that acting counter-dispositionally uses self-control and thus should result in a state of ego depletion.

Self-control is believed to use a limited mental resource (Baumeister, 2002; Baumeister, Vohs, \& Tice, 2007). There are multiple theories of self-control but the most prominent is termed the strength model of self-control. The model describes and conceptualizes self-control by means of a metaphoric muscle - thus the moniker (Vohs \& Baumeister, 2011). A muscle expends energy to exert force or accomplish tasks. Similarly, actively monitoring and modifying the self, termed self-regulation, uses mental resources. Like a muscle, once the resource is exhausted or depleted, it is fatigued for ensuing activities. This depleted state is termed cognitive fatigue or ego depletion. Rest, relaxation (Tyler \& Burns, 2008), and positive emotions (Tice, Baumeister, Shmueli, \& Muraven, 2007) are restorative thus the depleted mental state is typically short-term. Furthermore, similar to a muscle, the strength of the resource can be increased through practice and exercise (Baumeister, Gailliot, Dewall, \& Oaten, 2006). Baumeister and colleagues established that self-regulation exacts costs in the form of ego depletion (Baumeister et al., 1998; Muraven \& Baumeister, 2000; Muraven, Tice, \& Baumeister, 
1998; Tice et al., 2007; Vohs \& Faber, 2007; Vohs \& Heatherton, 2000; Vohs et al., 2005).

Self-control failures can be seen as the origin of many social and personal problems including poor health, substance abuse, self-defeating behaviours, interpersonal violence, underachievement, and obesity (Bauer \& Baumeister, 2011). These self-control failures have larger implications in that they can place a burden on society through social and economic costs. Perseverance on difficult tasks, delay of gratification (adhering to long-term objectives over short-term temptations), and more optimal self-presentation are linked to successful self-regulation (Bauer \& Baumeister, 2011; Baumeister et al., 2006). The positive consequences for successful self-control management and negative consequences for self-control failures has led to a substantial amount of research. Topics explored include types of depleting tasks (Hagger, Wood, Stiff, \& Chatzisarantis, 2010), methods for restoring the depleted self (Tice et al., 2007; Tyler \& Burns, 2008) and methods for increasing the strength of the resource and thereby minimizing cognitive fatigue and its possible costs (Baumeister et al., 2006; Muraven \& Slessareva, 2003).

Research on ego depletion traditionally employs a between subjects design with depleting and non-depleting tasks as conditions, followed by a comparison on a subsequent unrelated task. This procedure is known as the dual-task paradigm (Baumeister et al., 1998; Muraven et al., 1998). Control condition participants normally complete an easier version of the depleting task. For example, the cross-out letters task requires participants to cross-out all the occurrences of a particular letter in a passage of text. The depleting condition also requires participants to cross-out the same letter, in the same passage, but with the additional instruction to cross-out the letter only when it is 
adjacent to specific other letters or combinations. Therefore, the additional instruction introduces an inhibitory component to the activity. Furthermore, depleting conditions frequently change the instructions so there is a habit that needs to be overcome (e.g., starting with crossing out all of a particular letter and then switching to the inhibiting instructions). Depleting tasks include the white bear paradigm, affect regulation, crossout letters, Stroop, and decision making tasks. Unrelated tasks assessing ego depletion have included Stroop, persistence with anagrams, handgrip stamina, consuming bad tasting food or resisting good tasting food. All participants complete the same second unrelated task, such as a Stroop ${ }^{4}$ task where colour words are displayed on a computer screen and participants are required to indicate the colour of the font and not the meaning of the word (for example, the word blue written in green font.) Participants have to inhibit the automatic action to read the word to indicate the correct response (green). Poor performance on the second task, for the depletion condition relative to the control condition, is then evidence of ego depletion. Decision making, impression management, emotion regulation, resisting temptation, and thought suppression have all been shown to deplete the cognitive resource and thereby induce the state of ego depletion (for a review and meta-analysis of ego depletion, see Hagger et al., 2010). The term cognitive fatigue is more focussed than ego depletion in that the assessment of cognitive fatigue is limited to cognitive measures such as Stroop and mental arithmetic tasks.

A possible explanation for why introverts do not behave extraverted is that counter-dispositional behaviour uses self-regulation resources and thus causes ego

${ }^{4}$ Traditional Stroop tasks have participants say their responses out loud. The use of computers to record responses is frequently referred to as a modified Stroop task. 
depletion. Across numerous studies, modifying behaviour (e.g., impression management, suppressing emotional displays, modifying speech to remove um and err fillers, etc.) results in an ego depleted state (Muraven \& Slessareva, 2003; Vohs et al., 2005). Furthermore, non-habitual behaviour (conducting activities that are done infrequently) requires greater perceived effort than habitual behaviours (activities conducted daily, Gallagher et al., 2011). Moreover, when non-habitual behaviour is also counterdispositional, yet even greater effort is reported. That is, an individual may have to engage in an activity frequently (daily), thus it would be deemed habitual, while the activity may be congruent or incongruent with their disposition. Gallagher et al.'s results (2011) suggest an explanation for why introverts do not behave extraverted; greater effort is required for non-habitual counter-dispositional behaviour.

Zelenski et al. (2012) explored cognitive fatigue associated with acting out of character within the introversion-extraversion domain. They used the acting extraverted/acting introverted methodology in two studies. They posited that monitoring and modifying behaviour, in compliance with experimental instructions, would impact self-control resources and thus result in cognitive fatigue. Zelenski et al. (2012) assessed participants' performance on a Stroop task following the behavioural manipulation component in two separate studies. They found a significant interaction between condition and disposition. The worst Stroop performance, measured by response times, was for extraverts in the acting introverted condition (i.e., behaving counterdispositionally). There was no evidence of a depleted state for introverts in the acting extraverted condition, however. Thus, the pattern of results, across both studies, was asymmetrical as only one of the two counter-dispositional combinations was associated 
with cognitive fatigue (extraverts acting introverted).

Alternative measures of ego depletion have been employed in other acting studies and have included pre/post handgrip persistence (Gallagher et al., 2011), anagram completion (Williams, 2006), and errors in the game Operation (Gillen, 2009). Although these studies have failed to find support for ego depletion, methodological limitations can explain their lack of findings. Also, these alternative measures are more behavioural tasks, and it is also possible that depletion effects are limited to cognitive control tasks such as Stroop. Consistent with this idea, Gallagher et al. (2011) found greater effort with non-habitual behaviour, but not with their behavioural measure of ego depletion (handgrip persistence task). This further suggests the potential usefulness of including a measure of effort. On the other hand, dual-task paradigm studies typically show no differences in reported effort between depleting and non-depleting tasks (Muraven et al., 1998; Muraven \& Slessareva, 2003). Muraven and Slessareva conclude that "effortfulness is not a good measure of the amount of self-control required" (2003, p. 902). Considering these mixed findings for self-regulation measures, and to remain comparable to previous research, I included a measure of perceived effort in combination with Stroop to assess cognitive fatigue.

To summarize, across multiple studies, acting extraverted is associated with higher positive affect relative to both acting introverted and control conditions. Furthermore, greater positive affect is experienced by both trait introverts and trait extraverts. This diverges from the acting introverted condition where trait extraverts report the greatest effort, and show evidence for cognitive fatigue. In combination, these findings suggest asymmetrical consequences for acting counter-dispositionally. 
Zelenski et al. (2012) proposed that restorative positive affect associated with the acting extraverted condition counteracts the depleting effects for introverts acting extraverted (cf., Tice et al., 2007). Situational influences might also explain the unequal consequences of counter-dispositional behaviour as research demonstrates that behaving against situational norms is depleting (Vohs et al., 2005). Despite the number of studies conducted examining manipulated extraverted behaviour, the settings employed are highly similar. Across the acting studies, the normative behaviour is at least moderately extraverted. Therefore, the negative consequences for counter-dispositional behaviour identified so far are limited to behaviour that is also against the norm of the situations (i.e., introverted in an extraverted context).

\section{The Importance of Situations}

Situations clearly influence behaviour. Despite previous disagreement regarding the relative importance of situational factors versus personality factors in determining behaviour, commonly referred to as the person-situation debate, it is generally agreed that behaviour is influenced by both personality and situation factors and the interaction between them (Fleeson \& Noftle, 2009; Funder, 2006, 2009; Krueger, 2009; Lucas \& Donnellan, 2009; Mischel, 2009). Research exploring cross-context variations in behaviour have concluded that different situations produce different behaviours (Fleeson, 2007; Fleeson \& Leicht, 2006; Robinson, 2009) and situations can be powerful predictors of behaviour (Reis \& Collins, 2004).

Within the notion of situations predicting behaviour is the concept of situational strength (Michel, 1977). It acknowledges that personality influences behaviour but that the degree of influence is moderated by the strength of the situation, ranging between 
strong and weak. Strong situations do not readily permit variability in behaviour. For example, at a wedding ceremony people sit quietly and are attentive. There is minimal variability in behaviour, between people, due to personality. Differences in personality are more readily observed in weaker situations. For example, at a wedding reception some people will be dancing while others sit quietly at their table. These between person differences in behaviour may be indicative of differences in the personality (in the above example, the trait of extraversion). Despite this conceptual framework having intuitive appeal for understanding the influences between situations and personality, there is not a large body of empirical support (for a review see Cooper \& Withey, 2009).

Numerous other classification systems for situations have been proposed (see Yang, Read, \& Miller, 2009 for a review of situation taxonomies within social, personality and ecological psychology) although there is no agreed upon taxonomy of situations (Hogan, Harkness, \& Lubinski, 2000). Classifying features or characteristics of situations (e.g., pleasantness, success/failure, emotional involvement, etc.) and classifying the kind or type of situation (e.g., friends, work, school, travel, etc.) are the two basic procedures behind creating situation taxonomies. However, even among similar approaches to classifying situations (i.e., two taxonomies identifying situation features from a similar number of situations) the resulting taxonomies are distinct. Across the different situation taxonomies, however, there are some frequently occurring aspects. Therefore, there is progress and optimism that an agreed upon situation classification system can be achieved, although this is not the current state of affairs (Funder, 2013).

Overall, there is consensus that people vary their behaviour systematically with 
situational cues (Fleeson, 2001), behave more consistent between situations when situations are similar compared to different (Furr \& Funder, 2004), and within-person variability of behaviour can be partially explained by varying characteristics of situations (Fleeson, 2007). Experience sampling methodology shows that across situations, more extraverted behaviour is associated with greater positive affect (Fleeson \& Gallagher, 2009). Extraverted behaviour terms, such as talkative and assertive, require a social exchange and thus occur in social rather than non-social situations. The link between extraverted behaviour and positive affect is therefore unsurprising as social situations are more pleasant than non-social situations (Lucas \& Diener, 2001; Whelan \& Zelenski, 2012a). Surprisingly, despite the number of studies exploring manipulated extraverted behaviour (Fleeson et al., 2002; Zelenski et al., 2012), the situational contexts of these studies have been relatively homogenous. Therefore, the large similarity between situations may explain the similarity in findings across the acting studies.

The non-manipulated control condition serves as a benchmark-comparing behaviour in it to the acting conditions establishes if behaviour is more similar to the acting introverted or the acting extraverted condition. For example, average behaviour in a library would be considerably different from average behaviour at a party. Examining the level of extraverted behaviour of people, within a particular setting, is suggestive of the situational demands and if the normative behaviour of the situation is extraverted or introverted. That is, the average level of extraverted behaviour, when not manipulated, is suggestive of the normative behaviour for that situation.

In all studies that had control conditions, the level of extraverted behaviour between the acting extraverted condition and the control condition was less than the 
difference between control condition and acting introverted condition. Furthermore, the level of extraverted behaviour for the control condition is above the midpoint on a Likert scale (Whelan \& Zelenski, 2007; Zelenski et al., 2012). Additionally, naïve observers' ratings confirm that participants, with no instructions to modify behaviour, behave moderately extraverted (Gillen, 2009; Zelenski et al., 2012). Therefore, the situations of previous acting extraverted studies were at least moderately extraverted ${ }^{5}$. Consequently, the condition (acting introverted) associated with costs (ego depletion and greater effort) is the condition that requires behaviour to be incongruent to situational demands (behaving introverted in an extraverted situation).

People tend to behave consistent with situational norms (Pervin, 1968) and research has established that modifying behaviour so that it is contra-normative can result in ego depletion. Vohs et al. (2005) explored impression management to determine if having participants behave in a manner that was against situational norms (atypical selfpresentation) was depleting. In a series of between subjects studies, they asked participants to behave either in agreement with or in a manner that was uncharacteristic of the situation - behaving in an unfamiliar pattern of self-presentation was posited to require more self-regulation and thus would be depleting. Results supported predictions as they found depleting effects for contra-normative behaviour. Thus, these studies suggest that behaving contra-normatively has a depleting effect.

${ }^{5}$ Not all studies had control conditions (i.e., Fleeson et al., 2002; McNiel \& Fleeson, 2006), although their group discussion methodology was similar to that adopted by other studies. The group discussion format is assumed to be consistent across the studies and based on other studies (Whelan \& Zelenski, 2007; Zelenski et al., 2012) the group discussion setting is moderately extraverted. 
Considering the asymmetrical results of the acting studies, the consequences of cognitive fatigue and effort are limited to the condition that is contra-normative (i.e., acting introverted in an extraverted situation). Yet, interestingly, consequences are also limited to trait extraverts. Therefore, the acting findings (Fleeson et al., 2002; McNiel \& Fleeson, 2006; Zelenski et al., 2012) are explained equally well by the concepts of contra-normative and counter-dispositional behaviour. Unfortunately, these concepts (contra-normative behaviour and counter-dispositional behaviour) would also predict consequences that are not supported by the findings of the acting studies. Specifically, a contra-normative hypothesis would predict consequences for introverts in the acting introverted condition (i.e., a main effect for the acting introverted condition as it is contra-normative) whereas counter-dispositional hypothesis would predict consequences for introverts in the acting extraverted condition (as the behaviour is counterdispositional).

Both counter-dispositional and contra-situational concepts draw from selfregulation theory. Self-regulation posits that monitoring and modifying behaviour requires self-control resources and is thus depleting. Counter-dispositional concepts propose that behaving against one's disposition requires self-control resources whereas contra-situational concepts propose that behaving against situational norms requires selfcontrol. These concepts are not mutually exclusive. It stands to reason that behaving in a manner congruent with disposition should require less self-control, relative to counterdispositional behaviour. Similarly, behaving in agreement with situational norms would require less self-control, relative to contra-normative behaviour. In combination these concepts may fully explain the asymmetrical results of the acting studies. That is, the 
greatest costs are found when behaviour is both contra-normative and counterdispositional. Therefore, I propose that the effects found previously are due to selfregulation, and that personality buffers situational effects; negative consequences for contra-normative behaviours will be greatest when behaviour is also counterdispositional.

To empirically explore if self-regulation situational costs are buffered by personality, the consequences for acting out of character need to also occur in introverted situations, but rather than replicate previous research, the findings should be a mirror image. Research on self-regulation has not incorporated both disposition and situational concepts. The proposed research filled this gap by investigating differing consequences for acting counter-dispositionally in introverted situations. To explore consequences when acting out of character, the proposed studies examined positive affect, negative affect, effort, cognitive fatigue, and inauthenticity.

\section{The Present Research}

Neither contra-normative nor counter-dispositional concepts fully explain the asymmetrical patterns found thus far in acting research. Importantly, in combination, contra-normative and counter-dispositional concepts may fully explain the pattern of results. Thus, I propose that consequences for acting out of character would be greatest when behaviour is also against the situational norm. Therefore, the goal of this research was to explore the hypothesis that situational self-regulation costs are buffered by personality.

Similar to previous research on acting out of character, this research experimentally manipulated behaviour and assessed the affective and cognitive 
implications with three between-subjects conditions (acting extraverted, acting introverted and a control condition with no instructions to modify behaviour).

Dispositional extraversion was assessed prior to experimental sessions. Contrasting with previous research, these studies markedly differ in that the experimental settings were introverted. Thus, participants partook in activities where the normative behaviour was introverted but still allowed for variations in behaviours (i.e., participants in the acting extraverted condition were able to follow their instructions to modify behaviour). Measures of positive affect, negative affect, emotional valence, effort and Stroop performance (as a measure of cognitive fatigue) were assessed following the behaviour manipulation. These measures were identical, or highly comparable, to measures employed in previous acting studies to allow for comparisons of results. This research explored the affective and cognitive implications of behaving counter-dispositionally in two introverted settings ${ }^{6}$ to determine the importance of situational factors on previous findings of acting extraverted. Instead of replicating the asymmetrical pattern previously found, I proposed that the consequences for acting out of character would be a mirror image. Specifically, I posited that the greatest negative consequences would occur when behaviour is both contra-normative and counter-dispositional. In an introverted situation, acting extraverted would be contra-normative and therefore, the greatest costs of acting out of character were expected for introverts in the acting extraverted condition.

${ }^{6}$ This research did not explore situation variation with inferential statistics. A single study with both an introverted and extraverted situation requires extremely large sample size. Furthermore, the pattern of findings, for an extraverted situation, were not expected to differ from previous research. Thus, the prudent course of action was to establish if the pattern of previous studies would replicate or change in introverted situations. 
This research also expanded previous acting studies by incorporating a measure of authenticity. This allowed for authenticity to be explored as a possible outcome of behaviour, counter-dispositional or dispositionally-congruent. The previous association between authenticity and extraverted behaviour was strictly correlational with more extraverted behaviour linked to greater authenticity. Unfortunately, as previous acting studies have not included authenticity, the causal direction had yet to be established. That is, extraverted behaviour may cause authenticity or feeling authentic may cause extraverted behaviour. By manipulating state behaviour, I explored one causal direction, specifically if extraverted, or introverted, behaviour could cause feelings of authenticity. Still, it is important to note that a causal path going in one direction does not preclude it from also going in the opposing direction (i.e., the causal path may go in both directions).

In addition, by incorporating a measure of authenticity, I explored a novel concept, that feeling authentic is dependent on situational factors. Previous studies explored authenticity and extraverted behaviour employing experience sampling methodology. Conceivably, as no manipulations to behaviour occurred, it is probable that greater extraverted behaviour occurred in situations that were more extraverted (consider that people tend to behave consistent with situational norms). A possible explanation for the correlation between extraverted behaviour and authenticity is that people feel authentic when behaviour is congruent with situational norms. Supporting this notion are findings that authenticity and positive affect are correlated (Harter, 2002; Sheldon, Ryan, Rawsthorne, \& Ilardi, 1997) and social situations (i.e., more extraverted situations) are more pleasant than non-social situations (Lucas \& Diener, 2001; Whelan \& Zelenski, 2012a). By measuring authenticity following behaviour manipulations in introverted 
situations, I explored if authenticity is dependent on situation norms. That is, instead of greater extraverted behaviour predicting authenticity, I proposed that introverted behaviour would be linked to authenticity because the acting introverted condition is congruent with the situational norm. This contrasts with other views of authenticity that draw on true-self or personality factors in determining what is authentic. I proposed that situational factors would alter the pattern of findings from previous studies on state authenticity.

\section{Hypotheses}

Positive Affect. I hypothesized condition effects with participants in the control condition reporting the most positive affect and participants in the acting extraverted condition reporting the least (contra-situational). Within the control condition and the acting introverted condition, I did not expect differences in reported positive affect based on disposition (i.e., I expected the simple slopes to be flat). Although I expected participants in the acting extraverted condition to report the least positive affect, I expected this to be especially true for introverts. That is, I expected the simple slope for the acting extraverted condition to be positive. Therefore, I hypothesized an interaction between condition and disposition on positive affect. 


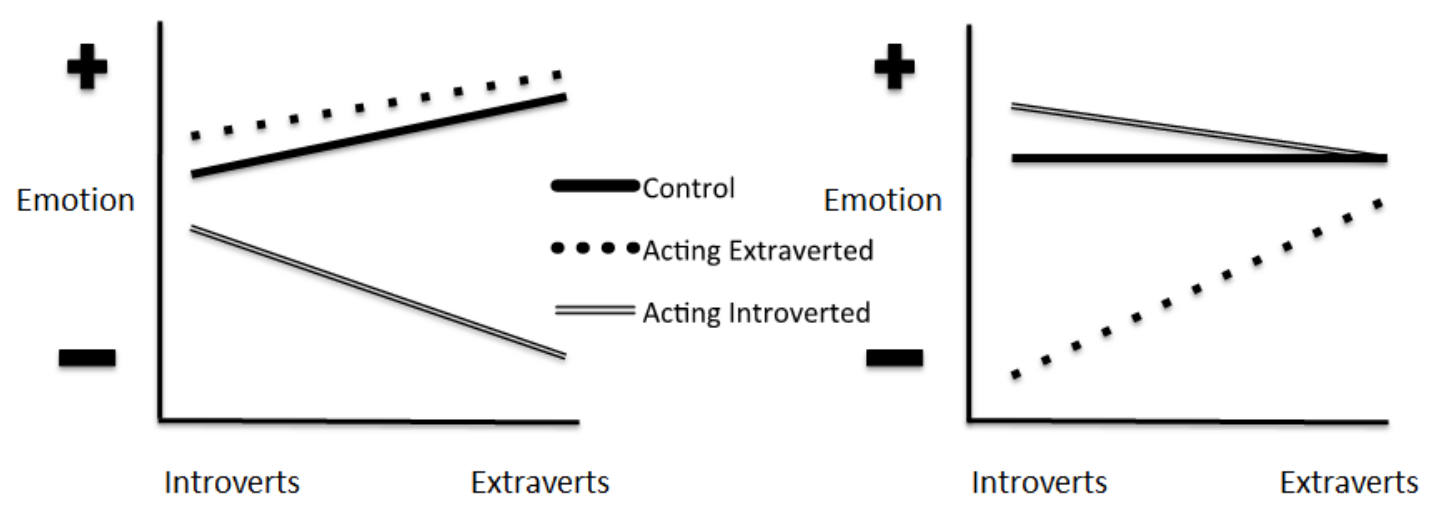

Figure 2. General pattern of trends of previous acting studies in the left panel, and the hypothesized trends for (unstudied) introverted situations in the right panel.

Valence. I hypothesized the same pattern of results for valence as I posited for positive affect.

Enjoyment. I did not expect condition effects or disposition effects for enjoyment of the activity. I hypothesized that there would be an interaction between condition and disposition on activity enjoyment. Specifically, I expected the simple slopes for control condition to be flat. I expected the simple slope for acting introverted to be negative and conversely, I expected the simple slope for the acting extraverted condition to be positive. That is, introverts in the acting introverted condition were expected to enjoy the activity relative to the extraverts in this condition whereas introverts in the acting extraverted condition were expected not to enjoy the activity relative to extraverts in this condition.

Negative Affect. No condition effects or disposition effects for negative affect were expected. I hypothesized an interaction of condition by disposition. Specifically, I 
expected the control and the acting introverted conditions simple slopes to be flat with regard to personality. Conversely, I expected the simple slope for acting extraverted condition to be negative. That is, I expected introverts to report more negative affect, relative to extraverts, but only when instructed to act extraverted.

Self-consciousness. I hypothesized the same pattern of results for selfconsciousness as I posited for negative affect.

Authenticity. I hypothesized a condition effect on authenticity such that participants in the control condition report the most subjective authenticity compared to both acting conditions, and with participants in the acting extraverted condition reporting the lowest level of authenticity. I did not expect reports of authenticity to differ by disposition within the control condition (i.e., the simple slope for the control condition is posited to be flat). I further hypothesized that people in the acting introverted condition would report greater subjective authenticity compared to people in the acting extraverted condition. Although I expected the introverted condition to produce more authenticity than the acting extraverted condition, I expected this to be especially true for trait introverts. That is, I hypothesized that the slope for acting introverted condition would be negative. I expected that extraverts would report more authenticity than introverts in the acting extraverted condition. That is, I hypothesized that the slope for acting extraverted condition would be positive. Thus, I also hypothesized an interaction of condition and disposition on authenticity.

Effort. I hypothesized a condition effect on effort such that participants in the control condition report the least amount of effort compared to both acting conditions. Within the control condition, I did not expect differences in reported effort based on 
disposition (i.e., I expected the simple slope for control condition to be flat). I also hypothesized that people in the acting extraverted condition would report more effort compared to people in the acting introverted condition (contra-situational). Although I expected the extraverted condition to produce greater effort, I expected this to be especially true for introverts. That is, I expected the acting extraverted simple slope to be negative.

For the acting introverted condition, I expected reported effort to be midway between acting extraverted and control conditions although I also expected that extraverts would report greater effort within this condition. That is, I expected the simple slope for acting introverted to be positive. Thus, I also hypothesized an interaction between condition and disposition on effort.

Cognitive Fatigue. I did not expect condition effects or disposition effects for Stroop performance. I hypothesized that there would be an interaction between condition and disposition on Stroop performance. Specifically, I expected the simple slopes for control and acting introverted conditions to be flat. Conversely, I expected the simple slope for the acting extraverted condition to be negative. That is, introverts in the acting extraverted condition were posited to perform worse relative to extraverts in this condition.

\section{Study 1}

\section{Method}

Participants. A total sample of 194 participants in 59 groups was collected. Participants' lack of following instructions required eliminating either the participant or the group. These decisions were based on the degree of influence on the other 
participants. Participants were eliminated when there was no perceived influence to the other group members (discrete use of cell phone while completing questionnaires) whereas groups were eliminated when there was obvious impact to the group dynamics (bathroom break during the puzzle task, mentioned their acting instructions during the activity, etc.). Five groups plus 5 participants were removed for issues involving not following instructions. Furthermore, due to missing/incomplete online trait data, 14 participants were removed. The final dataset for analyses consisted of 158 participants (49 males) run in 54 experimental sessions (40 groups of 3 participants and 14 groups of 4 participants).

Materials.

Personality traits. Goldberg's Markers (Goldberg, 1992) were used in an on-line questionnaire. The trait of extraversion was measured with 20 items. Participants responded on a seven-point Likert type scale for how much each adjective is representative of them in general with 1 indicating not at all to 7 very much. The mean of the items (bold, extraverted, shy [reversed], etc.) was calculated to form a dispositional extraversion score (Cronbach $\alpha=90){ }^{7}$ (Appendix A).

Affect. This questionnaire had a total of 18 adjectives rated on a seven-point Likert type scale. Participants self-reported how much you felt each adjective DURING the recently completed task with 1 indicating very slightly or not at all to 7 extremely or a lot. Similar to the Positive affect Negative Affect Schedule (PANAS, Watson et al.,

${ }^{7}$ Goldberg's full 20-item measure of extraversion (Goldberg, 1992) was used in conjunction with Saucier's Mini Markers (based on Goldberg's markers, Saucier, 1994) for the other four traits of neuroticism, openness, agreeableness, and conscientiousness. Only the trait of extraversion was considered under this line of inquiry. 
1988), a subset of high arousal positive and high arousal negative items were used in creating the positive affect and negative affect terms. The mean of excited, interested, strong, alert, and active, were used to create the composite positive affect term while irritable, upset, nervous, hostile, and distressed were used to create the composite negative affect term (Cronbach $\alpha=.83$ and .66 respectively). To more comprehensively explore emotional experience, emotional valence, arousal and self-consciousness were also assessed. Valence was created by averaging pleased, happy, unhappy* and sad* (Cronbach $\alpha=.73$ ), arousal was created by averaging alert, active, stimulated, tranquil*, idle $^{*}$, and quiet* (Cronbach $\alpha=.67$; items with an asterisk indicates reverse scored items), and self-consciousness was created by averaging self-conscious, worried, nervous, anxious, and ashamed (Cronbach $\alpha=.78$, Appendix B).

A similar measure of affect was collected prior to the experimental task but asked participants to forecast or predict their emotions during the activity (Appendix C). This questionnaire assessing affective forecasting was not examined under this line of inquiry.

Behaviour. To determine if participants followed instructions to modify behaviour and to establish the normative behaviour of the situation, state behaviour was assessed. The measure consisted of 18 items, six assessing extraverted behaviour. ${ }^{8}$ Participants indicated their level of agreement, for each adjective, that it was descriptive of how they behaved during the activity. Each item was rated on a seven-point Likert type scale with 1 indicating strongly disagree to 7 indicating strongly agree. Similar to Fleeson and Wilt (2010) the six adjectives assessing state extraverted behaviour were the

${ }^{8}$ The remaining twelve adjectives assess behavioural aspects associated with the other four personality traits. 
mean of energetic, assertive, talkative, shy*, bold, and quiet ${ }^{*}$ (Cronbach $\alpha=.86$, items with an asterisk indicate reverse scored items, see Appendix D).

Effort. This scale is designed to assess participants' effort during the activity. Statements are modelled after Gallagher et al.'s (2011). Modifications were made to the statements to remove references to instructions to act so that participants in the control condition would remain blind to the nature of the experiment. Participants indicate how much they agree with each of five statements, (e.g., It was effortful to behave the way I did during the activity) using a seven-point Likert type scale $(1=$ strongly disagree to $7=$ strongly agree, Cronbach $\alpha=.75$ ). (Effort and Authenticity items were combined into a single questionnaire for ease of administration, see Appendix E).

Authenticity. This scale was designed to assess participants' subjective authenticity and consists of ten items. The items were largely borrowed from Fleeson and Wilt (2010) and supplemented by items from a trait authenticity measure (AI3; Goldman \& Kernis, 2004). Items were modified so that they were statements that could be responded to on an agreement scale (different scales were used between Fleeson and Wilt's various studies). Participants indicated their level of agreement with statements (e.g., During the last 20 minutes, my behaviour represented who I really am) on a sevenpoint Likert type scale $(1=$ strongly disagree to $7=$ strongly agree, Cronbach $\alpha=.89$, see Appendix E).

Opinions about the activity. Seven questions comprised this scale to assess participants' attitudes about the activity. Averaging three items created the activity 
enjoyment score. ${ }^{9}$ All items align with those used by Fleeson et al., (2002) but the wording was modified from discussion to activity to reflect the different activities of this research. Participants indicated how much they agree with each statement (e.g., The activity was enjoyable) using a seven-point Likert type scale $(1=$ strongly disagree to $7=$ strongly agree, Cronbach $\alpha=.71$, see Appendix F)

Perception of other group members. Participants will rate how they perceived the other group members during the activity. This single page questionnaire assessed perceived affect and behaviour. Affect and behaviour were assessed with the identical adjectives as the self-report questionnaires but with two additional items. Participants were asked to indicate, on a seven-point Likert type scale, how well the adjective describes the other participant $(1=$ strongly disagree to $7=$ strongly agree $)$. The two additional items were to determine how confident participants were in their rating of the other participants. That is, participants may be overly absorbed in the activity and thus not have good knowledge of the behaviour and emotions experienced by the other activity members. The new questions were designed to explore this aspect ${ }^{10}$ (see Appendix G). The control condition was naïve to other participants receiving instructions to modify behaviour and thus provide a more objective, unbiased rating of extraverted behaviour and perceived affect. Control condition participants ratings of the other group members, termed observer-ratings, servers to confirm self-reports.

${ }^{9}$ The other questions pertained to previous knowledge of the other activity members and perceptions of the activity, separate from enjoyment, such as learning and boredom.

${ }^{10}$ Control participants' confidence ratings for behaviour was 5.87 and for affect was 5.75 . 
Perception of extraverted behaviour, positive affect, negative affect, arousal, valence, and self-consciousness were created with identical items as the self-report measures but were calculated using only control participants ratings (Cronbach $\alpha=.83, .84, .63, .63, .70$, and .79 respectively).

Stroop task. A computerized Stroop task was used to assess ego depletion. The task was run with SuperLab software, on eMacs, and used I/O response keyboards with colour-coded keys for responding. The task began with coloured circles displayed; participants had to indicate the colour of the circle. This step assessed colour-blindness with all five colours of the Stroop task (red, yellow, green, blue, and purple) being tested. This step also serves as a learning component where participants grasped the concept of responding to the colours presented on the screen. The main Stroop task presented colour words shown in various colours of print. Participants indicate the colour of the print or ink (e.g., red printed in blue would have the correct response of blue). The majority of trials were incongruent (word and print colour mismatch, $n=260$ ) with a minority of congruent trials (word and print colour match, $\mathrm{n}=65$ ). Trials are randomized and the 300 Stroop trials took approximately 5 minutes to complete. The response (correct or incorrect) and response time (in milliseconds) was recorded for each trail.

Response time data was cleaned (i.e., trimmed) with responses less than $300 \mathrm{~ms}$ or greater than 3 seconds being removed prior to analyses. Multiple outcome variables were created as Stoop performance can be assessed in a variety of ways. All outcome measures were created following trimming and include: the number of errors made, the average response time, the average response time for correct trials, the median response time, and an interference response time (response time of incongruent trials minus the 
response time of congruent trials). For all measures, higher numbers represent poorer performance.

Instruction to modify behaviour. The written instructions to modify behaviour were almost identical to those used by Fleeson et al (2002) and subsequent acting studies $^{11}$. Participants in the acting extraverted condition were told to behave bold, talkative, energetic, active assertive, and adventurous. Participants in the acting introverted condition were told to behave reserved, quiet, reflective, passive, compliant, and unadventurous. For clarity regarding the duration for which they are being instructed to modify their behaviour - the instructions note that they should behave in the manner described until they receive the next booklet of questionnaires at which point, they can be themselves for the remainder of the study. The instructions page was modified for this study to include a confirmation component. Specifically, a series of word pair adjectives were listed. Participants are asked to indicate (circle) the adjective that aligns with how they would behave in the upcoming activity (see Appendix $\mathrm{H}$ ).

Situation. The activity took place in a conference room located in the Carleton University Happy Lab (CUHL) space and directly across the hall from a series of small rooms. The blinds were drawn so that consequences of weather and seasonal views were minimized. Immediately inside the conference room there were two table arrangements. The first arrangement was a single table in close proximity to the door. There was space around the table such that people could comfortably walk around it. Note that there were

${ }^{11}$ In previous acting studies, the term lethargic was used to describe introverted behaviour. This term was changed to reflective as it was thought the term could be interpreted negatively. 
no chairs at this table. On this table, there was a jigsaw puzzle ${ }^{12}$. Photos of the puzzle image were placed beside the puzzle pieces. A second table arrangement, comprised of three tables forming a large rectangle, and was located against the far wall of the conference room. Tent labels (A through D) were on the table top and chairs were aligned with each label. Cardboard dividers, approximately 1 foot high, were placed between participants to assist in concealing responses. Participants were instructed that their activity was to try to complete the puzzle and that they had up to 20 minutes to achieve the objective.

\section{Procedure.}

This was a two-part study. Part one was a series of questionnaires completed online; part two was an experimental session conducted in a laboratory setting. The on-line questionnaires included measures of disposition (introversion-extraversion) and other questionnaires collected for separate lines of inquiry. ${ }^{13}$ The completion of the on-line questionnaire ensured that the assessment of extraversion was not proximal to asking participants to alter their extraverted behaviour. The on-line questionnaire was similar to using mass testing but did not limit participants to those who complete mass testing which was only available for approximately 1 month of the year.

${ }^{12}$ During pilot testing, having participants work on smaller individual puzzles resulted in participants in the acting extraverted condition not following instructions, such that there was no conversation during the 20 minute activity. I established that having participants work on a single puzzle as a group created the basis for interaction and conversation required by the acting extraverted condition while maintaining an introverted situation. Furthermore, pilot testing determined that a 200 to 300-piece puzzle was sufficiently challenging without being demotivating.

${ }^{13}$ Participants completed a trait authenticity scale (Wood, Linley, Maltby, Baliousis, \& Joseph, 2008), and BIS-BAS (Carver \& White, 1994) and rated their ideal self using Goldberg's Mini Markers (Saucier, 1994). 
Once participants signed-up for the experimental session, they were emailed the invitation code required to access the on-line study. When needed, reminder emails to complete the on-line questionnaire were sent prior to the scheduled lab sessions.

Experimental sessions were run with 3 or 4 participants per session and lasted just over 1 hour. Sessions were restricted to three or four members as dyadic interactions may be fundamentally different. Furthermore, previous research has focussed on three person interactions and therefore, to examine situational influences, consistency with previous study designs was desirable.

For sessions with three members, a single participant was assigned to each condition. When sessions had four members, a second participant was assigned to act introverted $^{14}$. Random assignment to condition (act extraverted, act introverted and control) was done prior to participants' arrival at the testing session.

Upon participants' arrival at the CUHL lab, each participant was lead to one of a series of small rooms. Each room was equipped with a computer desk, computer terminal, and chair. The isolation of participants prior to the beginning of the testing session was to prevent participants from getting to know the other group members. Once all group members had arrived (or a sufficient allotment of time for waiting for other members had expired), the experimental session began with completing an informed

${ }^{14}$ Originally, the plan was to have an additional control condition to allow for extra naïve ratings. After conducting a small number of sessions with 4 members, where 2 participants were assigned to the control condition, the design was amended as the situation occasionally shifted to be extraverted. A critical component of this research was to have an introverted situation thus the additional participant was assigned to act introverted and the large sessions conducted were considered pilot data and excluded from analyses. 
consent. Participants were then given the instruction sheet for their condition. The instruction page informed each participant what their label was (A, B, C, D) and that they needed to take note of their label. The researcher confirmed individually that participants understood the instructions and asked if they had any questions. Participants were then informed that their work/play activity was to solve a puzzle. Participants were then told that they had 20 minutes to work as a group and try to solve the puzzle. Participants then completed the affective forecasting of emotions and were then brought into the larger conference room and directed to the puzzle. After 20 minutes, participants were instructed to seat themselves at the large table configuration at the other end of the room. Participants were also instructed to seat themselves in front of the appropriate labels. Participants were then asked to complete a questionnaire booklet (emotions, behaviour, authenticity, effort and enjoyment of activity material were included in this booklet).

Participants then completed ratings of how they perceived the other group members (emotions and behaviour). For clarity, each other member rating questionnaire was on coloured paper that matched the coloured tent label of the individual being rated. For example, tent label B was printed on green paper. When group members rated the behaviour and emotions of participant $\mathrm{B}$, the rating sheets were also on green paper. Note that the ratings of the other participants were completed at the large table so that they could see who they were rating. This was to facilitate participants' ability to recall the behaviour of a particular participant. Participants rated all the other group members.

Once all participants had completed the ratings of the other group members, they were each brought back to the small private room where the study had begun. The computer stations were prepared to administer the Stroop task while participants 
completed the questionnaire booklets. Participants were given instructions, both orally by the researcher and visually on the computer screen, for how to complete the Stroop task. Participants were instructed to go as quickly and accurately as possible. The Stroop task ends with instructions on the screen telling the participant to inform the researcher that the task has ended. Participants were then debriefed and thanked for their time.

\section{Analyses}

Experimental sessions were run with groups of participants where participants interacted with others in their session. It is reasonable to assume that a participant would share more similar experiences with the group members from their experimental session compared to group members from different experimental sessions. That is, if session members were getting along and enjoying themselves, enjoyment and positive emotions would be more similar for members of that session compared to members belonging to a session where one or more participants were constantly commenting that they hated doing puzzles. Consequently, each participant was not completely independent as there was a relationship between shared experimental session participants. This shared experience due to study 1's design resulted in non-independence of cases. Regression and Analysis of Variance (ANOVA) require independence of cases and were thus not appropriate. Multilevel Modeling (MLM) is designed to analyse data where independence is violated (Raudenbush \& Byrk, 2002; Singer \& Willett, 2003). This study was analysed with MLM methodology. The program HLM, version 7, was selected and participants' scores (level 1) were nested within experimental sessions (level 2).

For all analyses, each model was assessed with a single dependent variable (DV). 
The DVs examined were positive affect, valence, enjoyment, negative affect, selfconsciousness, arousal, authenticity, effort, and Stroop performance. At level one, the final model (or analysis) included condition (two variables dummy coded to represent the three conditions with the comparison condition coded as the control condition with both variables entered uncentered), trait extraversion (grand mean centered), and the two interaction terms (cross product of centered trait extraversion by each dummy coded condition variable, the interaction terms were entered uncentered). See below for the full model.

For each DV, two models were compared (deviance statistics compared by $\chi^{2}$ with 1 degree of freedom with lower deviance indicating a better fit, Singer \& Willett, 2003). Using Full Maximum Likelihood, a model with trait extraversion varying (i.e., including $\mu_{1}$ in the model) was compared to a model where the variance for trait extraversion is fixed (i.e., removing $\mu_{1}$ from the model). A significant improvement to the model, by including $\mu_{1}$ (variability around trait extraversion), indicates that there is random fluctuation around the grand mean of trait extraversion between experimental sessions. Across all models, allowing trait extraversion to vary (including $\mu_{1}$ in the model) did not significantly improve model fit and the more parsimonious model was selected and reported. All subsequent analyses were conducted using Restricted Maximum Likelihood. 


\section{Level 1 Model}

$Y=\beta_{0}+\beta_{1} *($ Trait Extraversion $)+\beta_{2} *($ Act Extraverted $)+\beta_{3} *($ Act Introverted $)+$ $\beta_{4} *(\mathrm{ActE} \times \mathrm{TraitE})+\beta_{5} *(\mathrm{ActI} \times \mathrm{TraitE})+r$

\section{Level 2 Model}

$$
\begin{aligned}
& \beta_{0}=\gamma_{00}+\mu_{0} \\
& \beta_{1}=\gamma_{10}+\mu_{1} \\
& \beta_{2}=\gamma_{20} \\
& \beta_{3}=\gamma_{30} \\
& \beta_{4}=\gamma_{40} \\
& \beta_{5}=\gamma_{50}
\end{aligned}
$$

Multi-parameter tests (conceptually similar to examining $\Delta R^{2}$ ) were used to determine if there was a condition effect on the DV. Specifically, the multi-parameter tests were compared to a null model (no variables included) to determine if simultaneously adding $\beta_{2}$ and $\beta_{3}$ into the model significantly improve the model fit (Nezlek, 2011; Singer \& Willett, 2003). When there was a significant condition effect, the individual parameters were examined to determine which conditions differed (conceptually this is similar to examining post-hoc comparisons only when the omnibus test is significant). The impact of disposition was determined by examining its coinciding parameter for significance. A second multi-parameter test was used to determine if there is a significant condition $\mathrm{x}$ disposition interaction. Specifically, the multi-parameter test determined if simultaneously adding $\beta_{4}$ and $\beta_{5}$ significantly improve model fit. For significant interactions, the unique parameters were examined to explore the pattern of the interaction between the variables. And the simple slopes for each 
condition were determined (Aguinis, 2004).

\section{Preliminary Data Exploration}

To explore the relationships between the different dependent variables, bivariate correlations were computed. Table 1 displays the correlations between the ten selfreported dependent variables, Table 2 displays the correlations between the seven observer-rated dependent variables and Table 3 displays the correlations between the ten self-rated and seven observer-rated dependent variables. For both self-rated and observer-rated dependent variables, positive affect was significantly correlated with extraverted behaviour, valence and arousal; negative affect was significantly positively correlated with self-consciousness and negatively correlated with valence.

For the self-reported dependent variables, extraverted behaviour was significantly negatively correlated with effort. Extraverted behaviour was positively correlated with authenticity and enjoyment. In combination, extraverted behaviour may have been perceived as easy, enjoyable and authentic. There was a strong negative correlation between authenticity and effort. This may be partially due to the items of both scales being presented on the same questionnaire page.

For the observer-rated dependent variables, the single correlation of negative affect with extraverted behaviour was non-significant, all other correlations were significant. Of note were the positive correlations between observed agreeable behaviour with observed extraverted behaviour and valence. Participants that were perceived as more agreeable were also perceived as behaving more extraverted and experiencing more pleasant affect and less negative affect, whereas extraverted behaviour was more strongly associated with positive affect (high arousal pleasant affect) with no corresponding 
association with negative affect.

The correlations between self-rated and observer-rated paired dependent variables yielded mixed results. Importantly, there was a strong correlation between self-reported and observer-rated extraverted behaviour indicating good agreement. There were also positive correlations between pairings of positive affect and arousal whereas the pairings of agreeable behaviour, negative affect and self-consciousness were not correlated. Previous studies have also found poor agreement between self and observer ratings for negative affect; perhaps people are better at hiding, or more motivated to hide, their negative feelings. 
Table 1

Correlations Between Self-Reported Behaviour and Affect for Study 1

\begin{tabular}{|c|c|c|c|c|c|c|c|c|c|}
\hline & 1 & 2 & 3 & 4 & 5 & 6 & 7 & 8 & 9 \\
\hline 1. Extraverted Behaviour & - & & & & & & & & \\
\hline 2. Agreeable Behaviour & $.43 * * *$ & - & & & & & & & \\
\hline 3. Positive Affect & $.60 * * *$ & $.39 * * *$ & - & & & & & & \\
\hline 4. Negative Affect & -.16 & $-.20 *$ & -.07 & - & & & & & \\
\hline 5. Valence & $.45 * * *$ & $.49 * * *$ & $.62 * * *$ & $-.43 * * *$ & - & & & & \\
\hline 6. Arousal & $.73 * * *$ & $.44^{* * *}$ & $.79 * * *$ & $-.20 * *$ & $.54 * * *$ & - & & & \\
\hline 7. Self-consciousness & -.07 & -.06 & -.03 & $.79 * * *$ & $-.30 * * *$ & -.11 & - & & \\
\hline 8. Enjoyment & $.28 * * *$ & $.30 * * *$ & $.55 * * *$ & -.13 & $.56^{* * *}$ & $.38 * * *$ & -.02 & - & \\
\hline 9. Authenticity & $.58 * * *$ & $.47 * * *$ & $.46^{* * *}$ & -.12 & $.37 * * *$ & $.51 * * *$ & -.07 & $.29 * * *$ & - \\
\hline 10. Effort & $-.43 * * *$ & $-.42 * * *$ & $-.33 * * *$ & $.28 * * *$ & $-.40 * * *$ & $-.39 * * *$ & $.25 * *$ & $-.31 * * *$ & $-.72 * * *$ \\
\hline
\end{tabular}

Note: $* p<.05, * * p<.01, * * * p<.001$ 
Table 2

Correlations Between Observer-Rated Behaviour and Affect for Study 1

\begin{tabular}{|c|c|c|c|c|c|c|}
\hline \multicolumn{7}{|l|}{ Observer Ratings } \\
\hline & 1 & 2 & 3 & 4 & 5 & 6 \\
\hline 1. Extraverted Behaviour & - & & & & & \\
\hline 2. Agreeable Behaviour & $41 * * *$ & - & & & & \\
\hline 3. Positive Affect & $.71 * * *$ & $.63 * * *$ & - & & & \\
\hline 4. Negative Affect & -.14 & $-.46 * * *$ & $-.22 *$ & - & & \\
\hline 5. Valence & $.52 * * *$ & $.70 * * *$ & $.70 * * *$ & $-.43 * * *$ & - & \\
\hline 6. Arousal & $.67 * * *$ & $.57 * * *$ & $.77 * * *$ & $-.43 * * *$ & $.57 * * *$ & - \\
\hline 7. Self-consciousness & $-.31 * * *$ & $-.37 * * *$ & $-.32 * * *$ & $.79 * * *$ & $-.39 * * *$ & $-.46 * * *$ \\
\hline
\end{tabular}

Note: $* p<.05, * * p<.01, * * * p<.001$ 
Table 3

Correlations Between Self-Reported and Observer-Rated Behaviour and Affect for Study 1

\begin{tabular}{|c|c|c|c|c|c|c|c|}
\hline \multirow[t]{2}{*}{ Self-Ratings } & \multicolumn{7}{|c|}{ Observer Ratings } \\
\hline & 1 & 2 & 3 & 4 & 5 & 6 & 7 \\
\hline 1. Extraverted Behaviour & $.53 * * *$ & $.23^{*}$ & $.42 * * *$ & -.08 & $.32 * * *$ & $.47 * * *$ & -.08 \\
\hline 2. Agreeable Behaviour & $.21 *$ & .18 & $.21^{*}$ & -.05 & $.21^{*}$ & .16 & -.07 \\
\hline 3. Positive Affect & $.23 *$ & .10 & $.31 * * *$ & -.05 & $.24^{*}$ & $.30 * *$ & -.12 \\
\hline 4. Negative Affect & -.02 & .12 & .02 & -.08 & .17 & .06 & -.10 \\
\hline 5. Valence & .16 & .12 & $.21 *$ & -.12 & .10 & $.22 *$ & -.06 \\
\hline 6. Arousal & $.41 * * *$ & .11 & $.40 * * *$ & -.02 & $.24 *$ & $.38 * * *$ & -.16 \\
\hline 7. Self-consciousness & .07 & $.19^{*}$ & .04 & -.07 & $.20 *$ & .12 & -.16 \\
\hline 8. Enjoyment & .06 & .18 & .14 & -.08 & .16 & .14 & -.02 \\
\hline 9. Authenticity & .14 & .14 & $.20 *$ & -.06 & .19 & $.19 *$ & -.07 \\
\hline 10. Effort & -.07 & .02 & -.03 & -.04 & .08 & -.05 & -.01 \\
\hline
\end{tabular}

Note: $* p<.05,{ }^{* *} p<.01, * * * p<.001$, Self-observer matched correlations are bolded. 


\section{Manipulation Checks}

A manipulation check was conducted to determine if participants complied with acting instructions and if behaviour was influenced by disposition. There was a condition effect on extraverted behaviour, $\chi^{2}(2)=166.28, p<.001$, indicating at least one condition differed from another. Post-hoc comparisons revealed that all three conditions differed from each other at $p<.001$ with participants in the acting extraverted condition reporting the most extraverted behaviour, $M=4.41,{ }^{15}$ and participants in the acting introverted condition reporting the least, $M=2.01$. The control condition reported levels of extraverted behaviour that were midway between the two acting conditions but slightly skewed towards the acting extraverted condition, $M=3.67$. Thus, it appears that the acting instructions created the appropriate differences between conditions for selfreported behaviour. A condition by disposition interaction was tested on extraverted behaviour to explore the potential for behaviour to differ due to agreement/disagreement between disposition and acting condition. There was a significant condition by disposition interaction, $\chi^{2}(2)=10.35, p=.006$. Both the control and acting extraverted conditions' simple slopes were positive, $b=.44, S E=.16, p=.005 ; \mathrm{b}=.45, S E=.20, p$ $=.03$ respectively, indicating more extraverted participants behaved more extraverted in these conditions. The simple slopes between the acting extraverted and control conditions did not significantly differ from each other. The simple slope for the acting introverted condition was non-significant indicating that both dispositional introverts and

${ }^{15}$ All analyses are conducted using HLM software (7.0). In keeping with multilevel modeling traditions, estimated means will be reported. Coefficients reported are from "robust" estimations. 
dispositional extraverts behaved similarly within the acting introverted condition, $b=-.18$, $S E=.16, p=.28$, see Figure 3 .

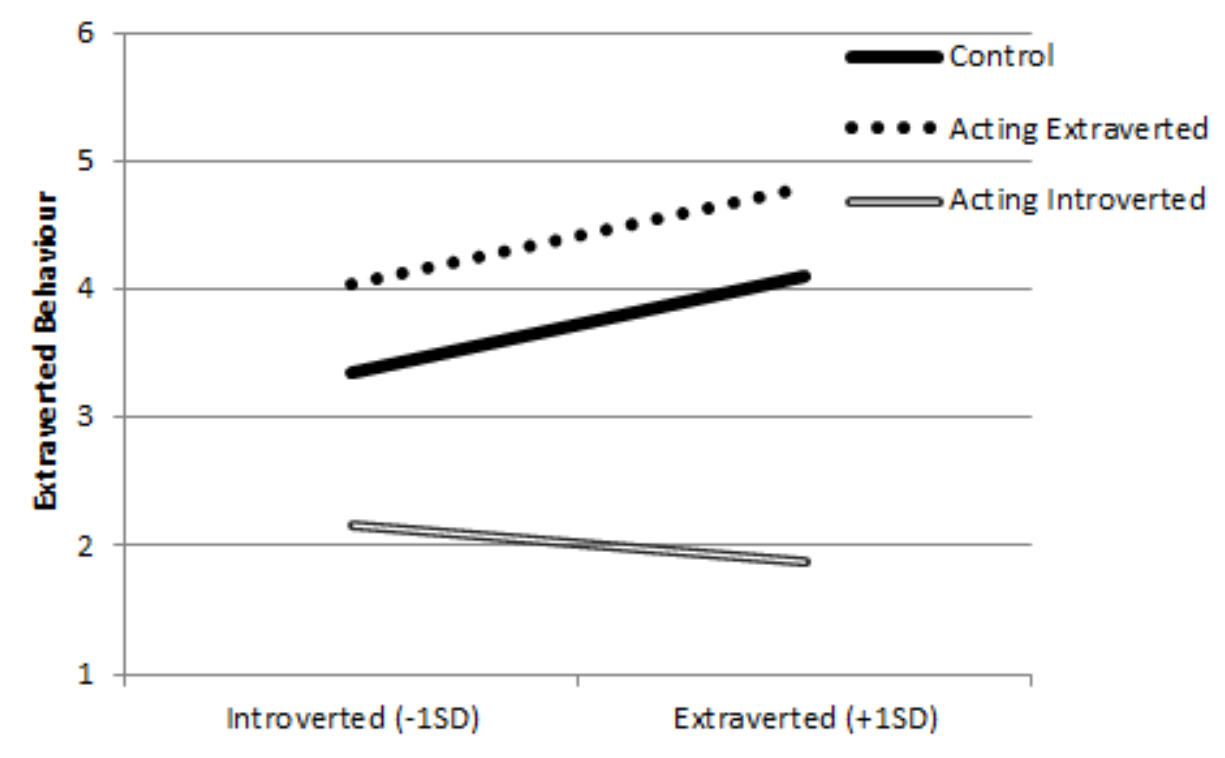

Figure 3. Disposition by condition interaction on extraverted behaviour.

A second form of manipulation check is to examine how other participants perceived fellow group members. Overall, the observer-rated level of extraverted behaviour indicates a condition effect, $t(129)=5.99, p<.001$, with participants in the acting extraverted condition $(M=4.40)$ perceived as behaving more extraverted than those in the acting introverted condition, $M=3.01$ (recall that the controls rated themselves right between these conditions at $M=3.67)$. Thus, observer-reports demonstrate that participants followed instructions. Targets' disposition was marginally related to how observers perceived participants' extraverted behaviour, $b=.24, S E=.13$, $p=.08$, indicating that across conditions, dispositional extraverts were perceived as behaving more extraverted. There was no interaction between targets' disposition and condition for how observers rated participants' levels of extraverted behaviour, $b=-.22$, 
$S E=.30, p=.46$. Although there was one unexpected interaction, overall, it appears that participants followed instructions to modify behaviour.

A key objective of this study is to create a situation that is introverted to allow for exploration of a situational effect compared to previous acting studies. I argued that previous acting research was conducted using extraverted situations. For comparison, Zelenski et al.'s (2012) grand means of extraverted behaviour are 4.29 and 4.14, for study 1 and 2 respectively, which are slightly above the midpoint on a 7-point Likert scale (Cohen's $d=.19$ and .15 from midpoint respectively). For this study, the overall estimated grand mean of extraverted behaviour is $3.25, t(157)=6.38, p<.001$, Cohen's $d$ $=.51$, significantly below midpoint, indicating a moderately introverted situation. To determine if the study mean is biased due to sessions conducted with 4 participants, where two members were instructed to act introverted, the mean of extraverted behaviour was calculated separately for 3-person and 4-person sessions. Both means indicated that the situation was normatively introverted $(M=3.28$ and $M=3.18$ for 3-person and 4person sessions respectively).

Recall that the level of extraverted behaviour for the control condition was also used to establish that the previous acting studies were moderately extraverted situations. For this study, the average level of extraverted behaviour in the control condition 3.67 (Cohen's $d$ for control condition versus midpoint is .27). This establishes that participants in the control condition behaved more introverted in the group puzzle situation than for the activities of previous acting studies although the effect is small. The levels of self-reported extraverted behaviour for all conditions are more introverted relative to previous research (this study $M=4.41,3.67$, and 2.01 for acting extraverted, 
control and acting introverted respectively compared to Zelenski et al.'s, 2012, Study $1 M$ $=5.62,4.96$, and 2.26 and Study $2 M=5.29,4.46$, and 2.67 for the three conditions respectively). Comparing this study to previous research, there seems to be a 1-point drop in extraverted behaviour for the acting extraverted and control conditions but a much smaller shift for the acting introverted condition.

Overall, the manipulation checks suggest that participants followed instructions and comparisons to previous acting studies suggest that the situation was more introverted. ${ }^{16}$ Although this situation may still have drawbacks to testing the combination of contra-situational and counter-dispositional behaviour, it seems that I created a normatively introverted situation in a lab setting.

\section{Results}

Hypotheses are motivated by the idea that both behaving in a counterdispositional manner and in a contra-situational manner will each lead to negative consequences. Furthermore, I argue that counter-dispositional and contra-situational behaviour costs can interact. Thus, hypotheses are derived assuming that the greatest negative consequences will be associated with a combination of both counterdispositional and contra-situational behaviour. I assume that the situation is introverted and thus contra-situational effects are expected for the acting extraverted condition. The greatest costs, combination of counter-dispositional and contra-situational, are hypothesized for introverts in the acting extraverted condition. Conversely, the least problematic combination is hypothesized to be for introverts in the acting introverted

${ }^{16}$ Despite the situation being more introverted than previous acting studies, there are indications that it was less than ideal, a point that will be addressed in the discussion. 
condition.

Positive Affect. Drawing on the general idea in the previous paragraph, I hypothesized a condition effect with participants in the acting extraverted condition reporting the least positive affect. I also hypothesized an interaction between disposition and condition with dispositional introverts, relative to dispositional extraverts, reporting even less positive affect. That is, the acting extraverted condition was hypothesized to have a positive simple slope (but not the other conditions). A condition effect was found $\chi^{2}(2)=23.64, p<.001$ where participants in the acting introverted condition reported the least positive affect compared to participants in both acting extraverted and control conditions (which did not differ significantly, see Table 4 for means by condition). Thus, the hypothesized difference of condition with acting extraverted participants having the least positive affect is not supported. There was a marginally significant disposition effect, $b=.18, S E=.11, p=.10$, indicating that as trait extraversion increases, so does reported positive affect. Contrary to my hypothesis, the interaction of condition by disposition was non-significant, $\chi^{2}(2)=2.11, p=.35$. Thus, acting introverted was less enjoyable, compared to both acting extraverted and control condition, and trait introverts did not differ from trait extraverts within conditions. For positive affect, not only was the interaction of disposition by condition hypothesis not supported, but the condition effect was opposite to that hypothesized.

Observer ratings of positive affect show a condition effect, $b=.86, S E=.18, p$ $<.001$, with participants in the acting introverted condition perceived as having less positive affect compared to participants in the acting extraverted condition (see Table 4). Targets' disposition and its interaction with condition were non-significant, $b=.02, S E$ 
$=.15, p>.50 ; b=-.13, S E=.27, p>.50$ respectively. Thus, observer-ratings are consistent with the self-reported positive affect condition trends, and both are contrary to hypotheses.

Valence. I hypothesized a condition effect with participants in the acting extraverted condition reporting the least positive valence. I also hypothesized an interaction between disposition and condition with the acting extraverted condition having a positive simple slope. There was a condition effect, $\chi^{2}(2)=12.92, p=.002$ with participants in the acting extraverted and control conditions reporting similar levels of valence and participants in the acting introverted condition reporting significantly lower valence. Thus, the hypothesized condition effect of participants in the acting extraverted condition reporting the lowest valence was not found. There was no disposition effect, $b$ $=.06, S E=.09, p>.50$, and the interaction of disposition by condition was also nonsignificant $\chi^{2}(2)=2.06, p=.36$. Thus, interaction hypotheses were not supported. Observer ratings of valence were consistent with self-reports; there was a condition effect, $b=.49, S E=.13, p<.001$, with the acting extraverted condition perceived as having higher valence than the acting introverted condition. Observer ratings also corroborate the non-significant influence of targets' disposition, $b=.01, S E=.10, p>.50$, and condition by targets' disposition interaction, $b=-.19, S E=.20, p=.36$.

Enjoyment. I hypothesized an interaction between condition and disposition on activity enjoyment. Extraverts in the acting introverted condition were expected to report less enjoyment relative to the introverts in that condition. Conversely, introverts in the acting extraverted condition were expected to report less enjoyment relative to extraverts in that condition. There was no condition effect, $\chi^{2}(2)=.82, p>.50$, or disposition effect, 
$b=-.04, S E=.13, p>.50$. The interaction between disposition and condition, $\chi^{2}(2)=.98$, $\mathrm{p}>.50$, was also non-significant. Therefore, how much participants enjoyed the activity did not depend on condition or their disposition.

Negative affect. Drawing on the idea that counter-dispositional and contra situational behaviour produces negative outcomes, I hypothesized an interaction of condition by disposition with the acting extraverted simple slope being negative. There was no condition effect, $\chi^{2}(2)=3.21, p=.20$, or disposition effect, $b=-.03, S E=.09, \mathrm{p}$ $>.50$ for negative affect. Contrary to hypotheses, the interaction of disposition by condition was non-significant $\chi^{2}(2)=1.73, p>.50$. Thus, the hypothesized disposition by condition interaction for negative affect was not found.

Interestingly, observer-ratings indicated a marginally significant condition effect, $b=-.17, S E=.10, p=.09$, with participants in the acting introverted condition, $M=2.01$, perceived as experiencing more negative affect relative to those in the acting extraverted condition, $M=1.83$. Targets' disposition did not predict observer-ratings of negative affect, $b=-.08, S E=.09, p=.40$, indicating that observer-ratings did not differ due to participants' trait extraversion. There was a significant interaction of condition by targets' disposition for observer-ratings of negative affect, $b=.25, S E=.11, p=.03$, see Figure 4 . For the acting introverted condition, as targets' trait extraversion increases, participants are perceived as experiencing less negative affect, $b=-.20, S E=.11, p=.07$. For acting extraverted there is no significant relationship between perceived negative affect and targets' disposition (i.e., the simple slope is flat, $b=.05, S E=.10, p>.50$ ). Despite the significant observer-rated negative affect interaction, the hypothesized direction of a negative slope for acting extraverted was not found. 


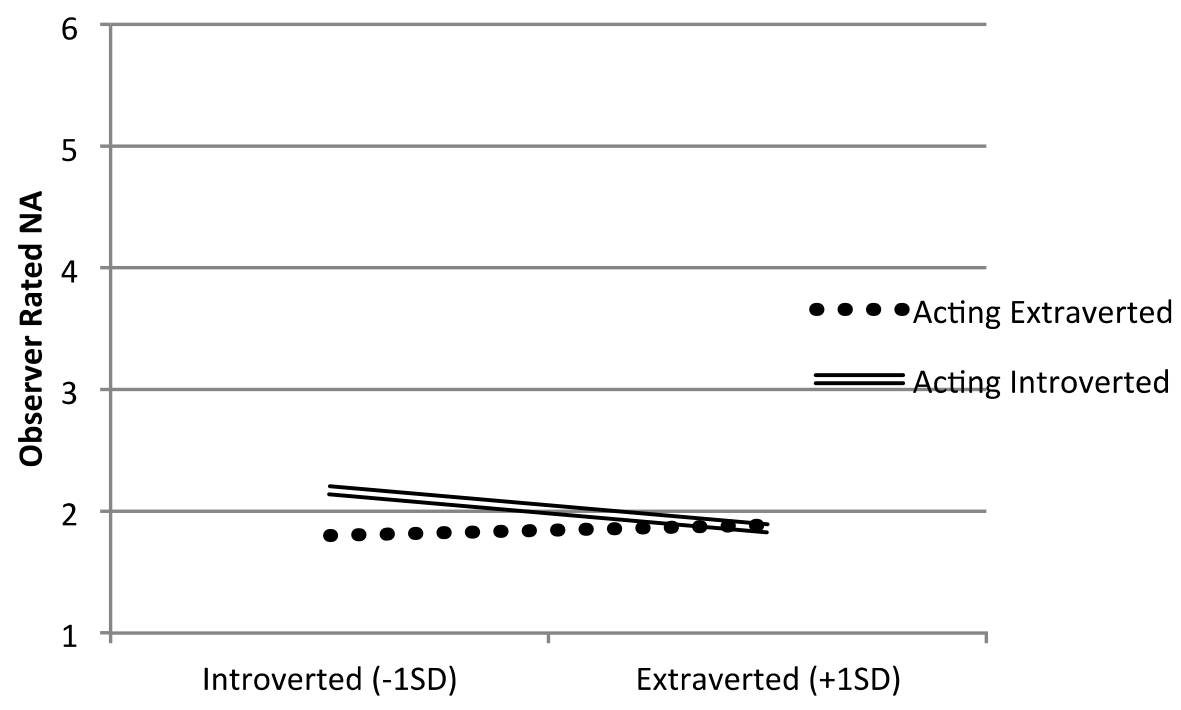

Figure 4. Interaction of condition by disposition for observer-rated negative affect.

Self-consciousness. I hypothesized an interaction of condition by disposition with the acting extraverted simple slope being negative. There was a marginally significant condition effect $\chi^{2}(2)=4.83, p=.09$ with participants in the acting extraverted condition reporting more self-consciousness than participants in the control condition but not differing from participants in the acting introverted condition. There was also a disposition effect, $b=-.29, S E=.10, p=.005$, indicating that trait extraverts report less self-consciousness compared to introverts. The interaction between disposition and condition was not significant, $\chi^{2}(2)=0.34, p>.50$. Thus, the hypothesized condition by disposition interaction was not found. The marginally significant condition effect for greater self-consciousness in the acting extraverted condition is revealing as a potential reason for why introverts do not act extraverted more frequently especially given their greater report of self-consciousness across conditions. 
Observer ratings reveal a condition effect, $b=-.31, S E=.14, p=.02$, with participants in the acting introverted condition perceived as more self-conscious than participants in the acting extraverted condition. There was no effect of targets' disposition, $b=-.05, S E=.12, p>.50$, but there was a marginally significant condition by targets' disposition interaction for observer-rated self-consciousness, $b=.39, S E=.21$, $p=.06$, see Figure 5. Both acting condition simple slopes are non-significant but have opposite relationship with targets' disposition. The acting extraverted simple slope indicates that as targets' trait extraversion increases there is a slight increase in perceived self-consciousness, $b=.15, S E=.12, p=.19$. The acting introverted simple slope indicates that as targets' trait extraversion increases there was a decrease in perceived self-conscious, $b=-.23, S E=.17, p=.17$.

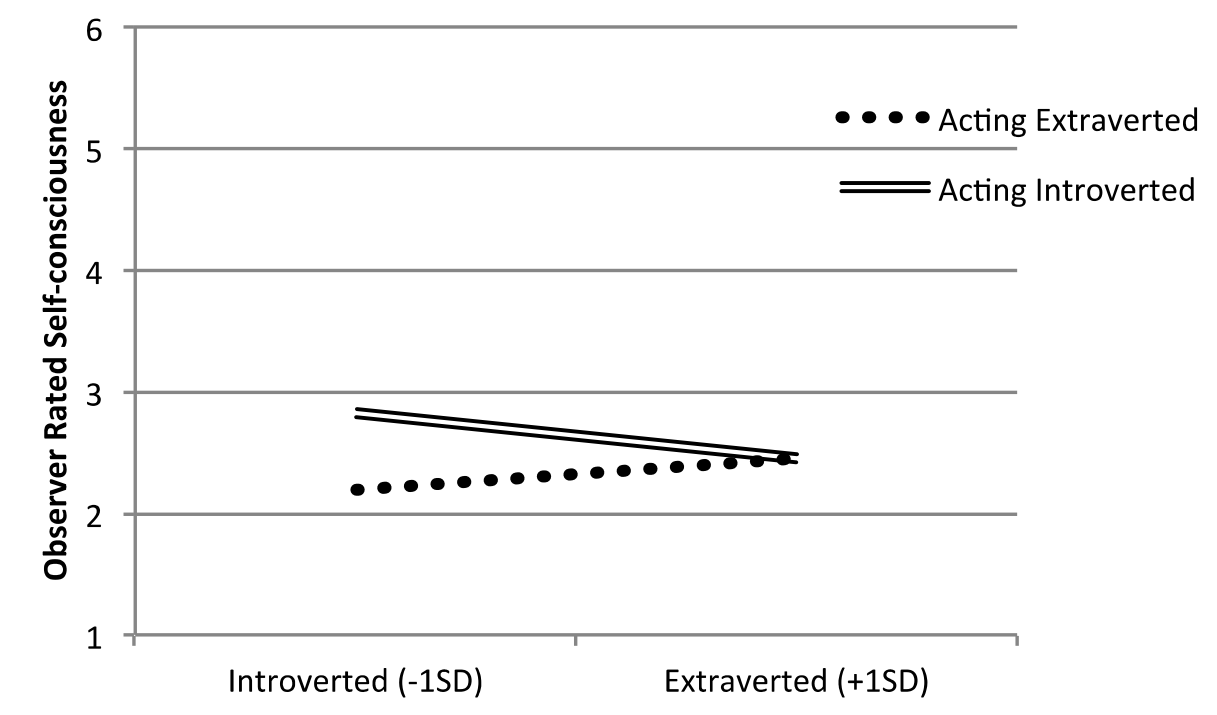

Figure 5. Interaction of condition by disposition for observer-rated self-consciousness.

Arousal. An exploratory analysis was conducted on arousal. There is a significant condition effect, $\chi^{2}(2)=67.84, p<.001$, with participants in the acting extraverted condition reporting the highest arousal and those in the acting introverted 
condition the lowest. There was no disposition effect, $b=.11, S E=.11, p=.30$ indicating that differences in arousal were not due to trait extraversion. There is a significant interaction of disposition by condition $\chi^{2}(2)=7.08, p=.03$. The acting extraverted simple slope is significantly positive, $b=.46, S E=.15, p=.003$, indicating that as trait extraversion increases more arousal is reported. The acting introverted, $b=-$ $.12, S E=.15, p=.42$, and control simple slopes are both non-significant, $b=.17, S E$ $=.14, p=.22$, see Figure 6 , indicating that trait introverts and trait extraverts reported similar levels of arousal within these conditions.

Observer ratings of arousal are consistent with the self-reported condition effect, $b$ $=.77, S E=.17, p<.001$, with those acting extraverted perceived as experiencing more arousal. The targets' disposition effect, $b=.07, S E=.12, p>.50$, and the interaction of condition by targets' disposition, $b=.07, S E=.23, p>.50$, were non-significant.

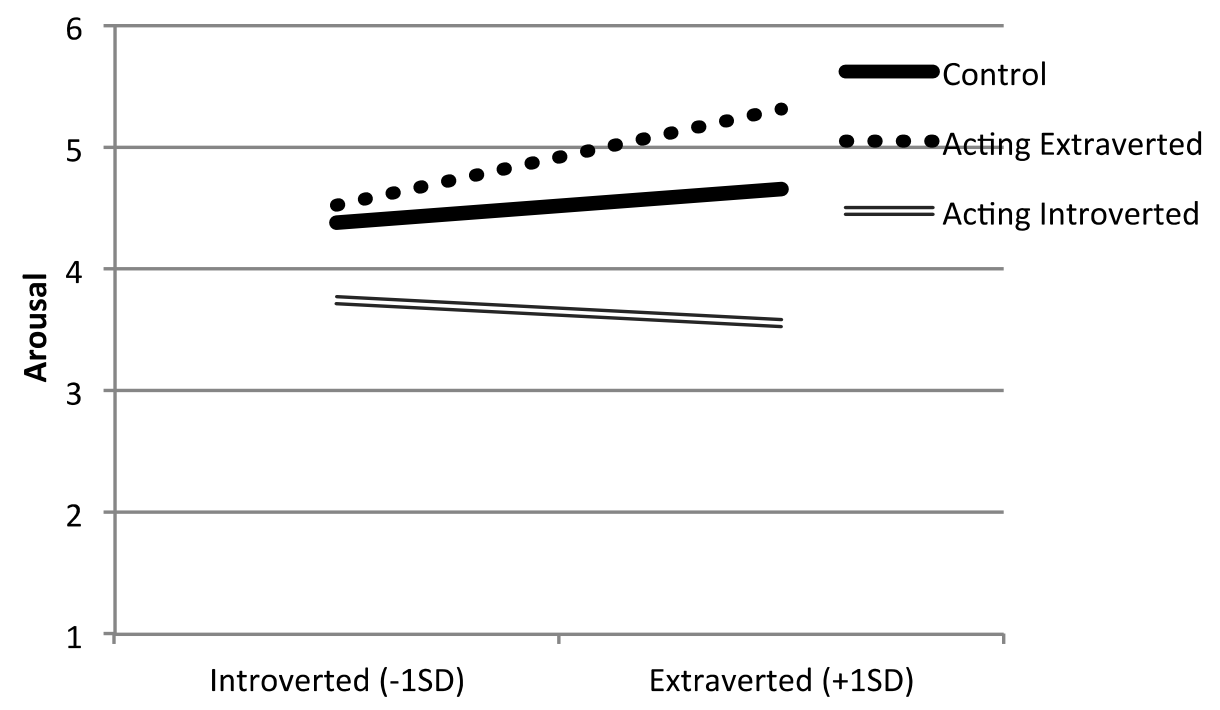

Figure 6. Interaction of condition by disposition for arousal.

Authenticity. Because the experimenter mandates acting conditions, I hypothesized a condition effect with participants in the control condition reporting the 
most authenticity and participants in the acting extraverted condition the least. I also expected an interaction between disposition and condition with introverts in the acting introverted condition reporting more authenticity than extraverts, and extraverts in the acting extraverted condition reporting more authenticity than introverts. There was a significant condition effect on subjective authenticity, $\chi^{2}(2)=130.23, p<.001$, where participants in the control condition reported feeling the most authentic, followed by participants in the acting extraverted condition (which did not differ from control). Participants in the acting introverted condition reported feeling the least authentic and were different from those in both other conditions. There was partial support of hypothesis of a condition effect on authenticity; participants in the control condition reported the most authenticity but participants in the acting extraverted condition reported more authenticity than participants in the acting introverted condition. The effect of disposition was non-significant, $b=.06, S E=.11, p>.50$, but disposition did interact with condition, $\chi^{2}(2)=37.13, p<.001$.

The pattern of the interaction was that both acting extraverted and control conditions have positive slopes, $b=.46, S E=.17, p=.006 ; b=.34, S E=.16 p=.04$ respectively, indicating that as trait extraversion increases, greater subjective authenticity is reported for these conditions. For acting introverted, there was a steep negative slope, $b=-.60, S E=.14, p<.001$, indicating that extraverts in the acting introverted condition reported significantly less subjective authenticity compared to introverts in the acting introverted condition. Thus, the direction of the simple slopes supports hypotheses. Interestingly, as Figure 7 demonstrates, at one standard deviation below the mean of trait extraversion (i.e., for introverts), the acting introverted condition was associated with less 
authenticity than both control and acting introverted conditions. Based on the figure, it appears that the interaction was driven by the acting introverted condition relative to the other two conditions (whose slopes do not differ). Authenticity hypotheses are partially supported. The simple slopes were in the hypothesized directions, but the hypothesis that the acting extraverted condition would produce the least authenticity was not found.

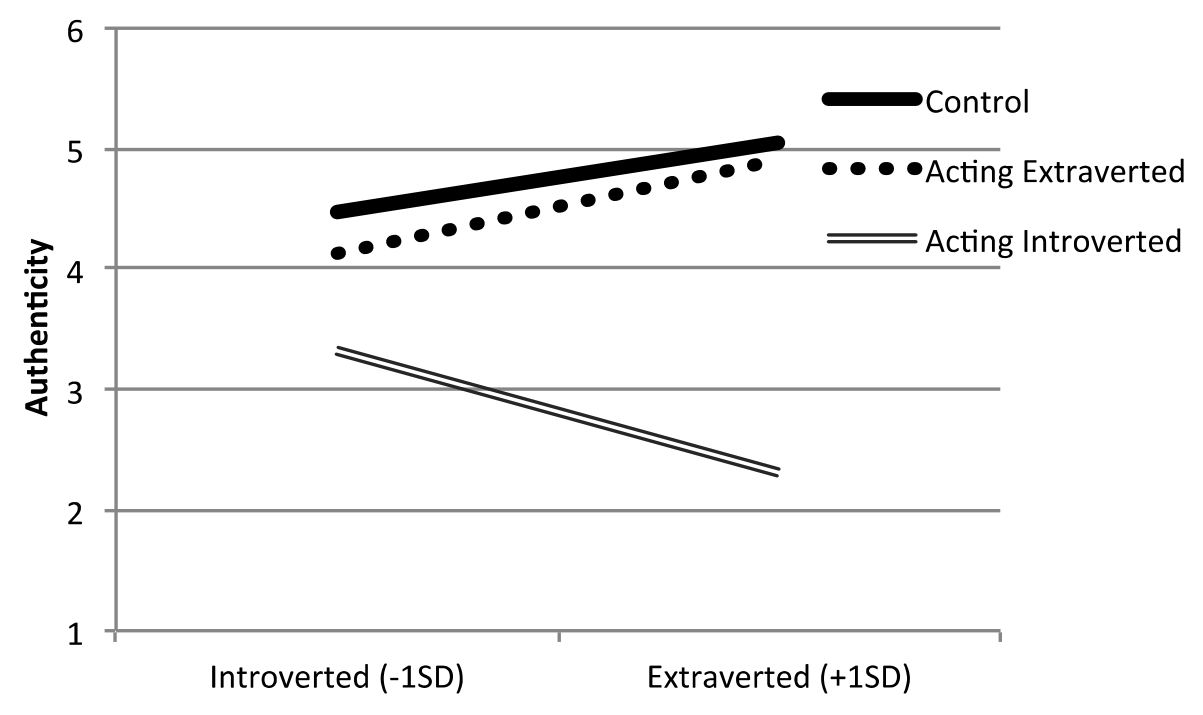

Figure 7. Condition by disposition interaction for subjective authenticity.

Effort. I hypothesized a condition effect with acting extraverted participants reporting more effort than the other conditions. I also expected that trait introverts in the acting extraverted condition would report more effort than extraverted participants.

There was a significant condition effect on effort, $\chi^{2}(2)=43.77, p<.001$. Participants in the control condition reported the least effort and, contrary to hypotheses, participants in the acting introverted condition reported the greatest amount of effort. No disposition effect of disposition was found, $b=-.13, S E=.13, p=.33$. There was a significant interaction of condition by disposition, $\chi^{2}(2)=22.79, p<.001$, see Figure 8 . The pattern 
of the interaction was that the control condition had a non-significant slope, $b=-.29, S E$ $=.17, p=.10$, whereas acting extraverted had a negative slope, $b=-.78, S E=.21, p$ $<.001$ (as trait extraversion increases less effort was reported for acting extraverted, i.e., it was easier for extraverts), and acting introverted has a steep positive slope, $b=.58, S E$ $=.19, p=.008$ (as trait extraversion increases more effort was reported for acting introverted, i.e., it was harder for extraverts to act introverted). The simple slopes of effort support hypotheses although interestingly, at 1 standard deviation below the mean, the effort for acting introverted and acting extraverted are indistinguishable.

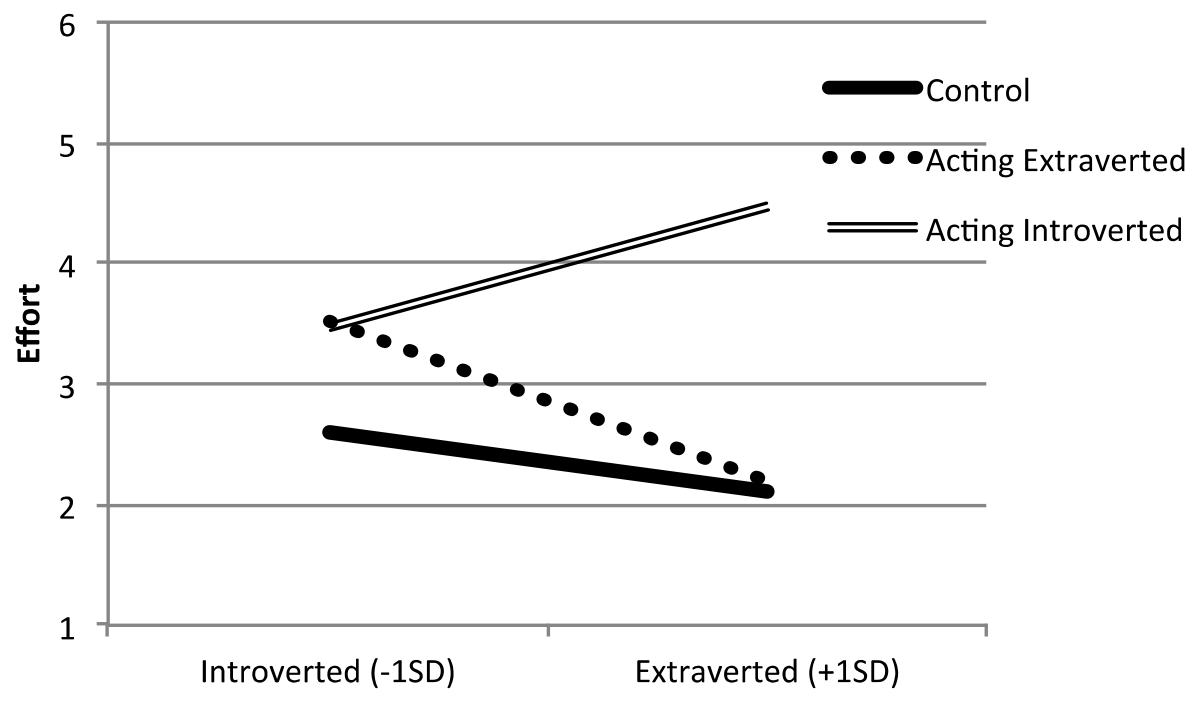

Figure 8. Condition by disposition interaction for effort.

Cognitive Fatigue. To determine if there were counter-dispositional or contrasituational effects on cognitive fatigue, Stroop performance was examined. Similar to the affect analyses, condition effects and disposition effects were examined followed by testing the interaction of disposition and condition. The number of errors made ranged from zero to 55 for untrimmed data and from zero to 48 for trimmed. Stroop performance was hypothesized to have an interaction between condition and disposition 
with the simple slopes for both acting introverted and control being flat and the simple slope for acting extraverted being negative (indicating that introverts in the acting extraverted condition performed worse than extraverts in that condition). Recall that Stroop can be examined in a number of ways, but across all methods the results were non-significant and no p-value approached significance (see Table 5). Thus, there were no cognitive effects found for counter-dispositional or contra-situational behaviours, nor their interaction. Cognitive hypotheses were not supported. 
Table 4

Estimated Means by Experimental Condition Across all Dependent Variables for Study 1

\begin{tabular}{llll}
\hline Dependant Variable & $\begin{array}{l}\text { Acting } \\
\text { Extraverted }\end{array}$ & Control & $\begin{array}{l}\text { Acting } \\
\text { Introverted }\end{array}$ \\
\hline Extraverted Behaviour & $4.41_{\mathrm{a}}$ & $3.67_{\mathrm{b}}$ & $2.01_{\mathrm{c}}$ \\
Observer Extraverted Behaviour & $4.41_{\mathrm{a}}$ & & $3.01_{\mathrm{b}}$ \\
Positive Affect & $4.59_{\mathrm{a}}$ & $4.34_{\mathrm{a}}$ & $3.64_{\mathrm{b}}$ \\
Observer Positive Affect & $4.73_{\mathrm{a}}$ & & 3.86 \\
Negative Affect & $2.07_{\mathrm{a}}$ & $1.86_{\mathrm{a}}$ & $2.20_{\mathrm{a}}$ \\
Observer Negative Affect & $1.83_{\mathrm{a}}$ & & $2.01_{\mathrm{b}}$ \\
Valence & $5.42_{\mathrm{a}}$ & $5.44_{\mathrm{a}}$ & $4.88_{\mathrm{b}}$ \\
Observer Valence & $5.60_{\mathrm{a}}$ & & $5.12_{\mathrm{b}}$ \\
Arousal & $4.90_{\mathrm{b}}$ & $4.51_{\mathrm{a}}$ & $3.65 \mathrm{c}$ \\
Observer Arousal & $4.99_{\mathrm{a}}$ & & $4.22_{\mathrm{b}}$ \\
Self-Consciousness & $2.66_{\mathrm{b}}$ & $2.22_{\mathrm{a}}$ & $2.47_{\mathrm{ab}}$ \\
Observer Self-Consciousness & $2.31_{\mathrm{a}}$ & & $2.62_{\mathrm{b}}$ \\
Authenticity & $4.52_{\mathrm{a}}$ & $4.72_{\mathrm{a}}$ & $2.77_{\mathrm{b}}$ \\
Effort & $2.86_{\mathrm{a}}$ & $2.39_{\mathrm{b}}$ & $4.01 \mathrm{c}$ \\
Enjoyment & $4.96_{\mathrm{a}}$ & $5.04_{\mathrm{a}}$ & $4.82_{\mathrm{a}}$ \\
Stroop Errors & $7.33_{\mathrm{a}}$ & $6.10_{\mathrm{a}}$ & $7.02_{\mathrm{a}}$ \\
Stroop Response Time & $925.64_{\mathrm{a}}$ & $892.31_{\mathrm{a}}$ & $913.71_{\mathrm{a}}$ \\
Stroop RT Correct & $923.63_{\mathrm{a}}$ & $891.50_{\mathrm{a}}$ & $912.71_{\mathrm{a}}$ \\
Stroop Median RT & $844.99_{\mathrm{a}}$ & $822.08_{\mathrm{a}}$ & $843.14_{\mathrm{a}}$ \\
Stroop Interference RT & $57.14_{\mathrm{a}}$ & $59.85_{\mathrm{a}}$ & $58.33_{\mathrm{a}}$ \\
\hline & & & \\
Note estimatedmeans & & & \\
\hline
\end{tabular}

Note: estimated means with different subscripts are different at least at $p<.05$ 
Table 5

Multi-Parameter Tests and Hypothesis Testing Results for Condition Effect, Disposition Effect, and Their Interaction Across Cognitive Dependent Variables for Study 1

\begin{tabular}{|c|c|c|c|}
\hline $\begin{array}{l}\text { Dependent } \\
\text { Variable }\end{array}$ & Condition effect & Disposition effect & $\begin{array}{l}\text { Condition by } \\
\text { Disposition } \\
\text { (Interaction) }\end{array}$ \\
\hline Errors & $\chi^{2}(2)=0.76, p>.50$ & $\begin{array}{l}b=.54, S E=.67, \\
p=.42\end{array}$ & $\chi^{2}(2)=.26, p>.50$ \\
\hline Mean RT & $\chi^{2}(2)=1.79, p>.50$ & $\begin{array}{l}b=11.01, S E=11.42 \\
p=.34\end{array}$ & $\chi^{2}(2)=0.96, p>.50$ \\
\hline Mean Correct RT & $\chi^{2}(2)=1.70, p>.50$ & $\begin{array}{l}b=11.54, \mathrm{SE}=11.36, \\
p=.31\end{array}$ & $\chi^{2}(2)=0.94, p>.50$ \\
\hline Median RT & $\chi^{2}(2)=1.40, p>.50$ & $\begin{array}{l}b=7.04, S E=9.10 \\
p=.44\end{array}$ & $\chi^{2}(2)=1.73, p>.50$ \\
\hline Interference RT & $\chi^{2}(2)=0.04, p>.50$ & $\begin{array}{l}b=5.09, S E=4.61, \\
p=.27\end{array}$ & $\chi^{2}(2)=0.82, p>.50$ \\
\hline
\end{tabular}

\section{Discussion}

Previous acting studies have employed moderately extraverted situations and found that acting extraverted was associated with more positive affect and this did not depend on disposition (i.e., even introverts enjoy acting extraverted). Furthermore, negative consequences (cognitive fatigue, effort) have been limited to extraverts in the acting introverted condition. This study sought to determine if the pattern of asymmetrical findings from prior acting studies were due to the moderately extraverted situations employed. Despite making the situational norm more introverted, yet similar to previous acting studies (Fleeson et al., 2002; McNiel \& Fleeson, 2006; Zelenski et al., 2012), participants in the acting extraverted condition reported more positive affect relative to participants in the acting introverted condition. This finding was corroborated by valence where participants in the acting extraverted condition reported feeling happier 
than participants in the acting introverted condition. Furthermore, there were no effects found for (self-reported) negative affect. Thus, despite adjusting the situational norm to be more introverted, the previous patterns of findings for affect were largely replicated by this study with acting extraverted being associated with more positive and no greater corresponding negative emotions. Thus, one might conclude that situations do not matter; that acting extraverted effects are robust across them.

However, there are other hints in the data that the more normatively introverted context made a difference. For example, condition effect sizes are much smaller for positive affect in this study relative to other acting studies (Zelenski et al., 2013 - Studies 2 through 4 compared to study 5 which is this dataset). Despite the hypothesized reversal of condition effects not occurring, the gap between the acting conditions was reduced. Furthermore, the affective findings were not corroborated by findings with the enjoyment scale. No differences for condition, disposition or their interaction were found in this study for enjoyment. Previous acting research has found that acting extraverted is reported as more enjoyable relative to acting introverted (Zelenski et al., 2013). The average level of enjoyment across conditions for the group jigsaw puzzle was approximately 5 on a 7-point scale ${ }^{17}$. Thus, the lack of findings for enjoyment was not due to the activity not being enjoyable. Comparing the average for this study to previous acting studies, participants in the acting introverted condition reported more enjoyment

${ }^{17}$ The mean across conditions for study 1 is 4.93 when calculated as described in methods. There are idiosyncratic differences in how enjoyment has been calculated between studies. The pattern holds regardless of which enjoyment items are used. To allow for direct comparisons of means, Zelenski et al., (2013) enjoyment was recalculated to match the methods described in this research. 
than in other studies (4.36 study 2, 4.24 study 3, 4.58 study 4, this study 4.82).

Conversely, participants in the acting extraverted condition reported less enjoyment than previous acting studies $(5.66,5.54,5.76$ for studies 2,3 and 4 respectively, this study 4.96). In combination, these findings demonstrate a shift in positive affect and enjoyment in the hypothesized directions, although the shifts were not sufficient to produce the hypothesized effects. That is, the difference in affective consequences of acting introverted versus extraverted appears to have been significantly reduced, but I did not observe a complete reversal between conditions. Acting extraverted became less positive but was still more positive than acting introverted despite the activity occurring in a more introverted situation.

This study also sought to expand on previous acting studies by exploring subjective authenticity following instructions to modify behaviour. Recall that Fleeson and Wilt (2010), using experience sampling methodology, found that participants reported greater authenticity when their behaviour was more extraverted and this did not differ by disposition. Even dispositional introverts reported feeling more authentic when their behaviour was more extraverted. In agreement with Fleeson and Wilt's results, I found that participants in the acting extraverted condition reported more authenticity compared to participants in the acting introverted condition. That is, across dispositions, there was a main effect of condition such that acting extraverted was reported as more authentic. In support of a counter-dispositional effect, as trait introversion increases, less subjective authenticity was reported for acting extraverted. That is, trait extraverts, relative to trait introverts, reported feeling more authentic when behaving extraverted. Similarly, as trait extraversion increases, less subjective authenticity was reported for 
acting introverted. That is, trait extraverts, relative to trait introverts, report less authenticity when behaving introverted. The contra-situational hypothesis was not supported; despite the situation being introverted, participants in the acting introverted condition reported less authenticity than participants in the acting extraverted condition. Fleeson and Wilt's (2010) finding that behaving extraverted feels authentic for both introverts and extraverts was novel. Study 1 findings replicate Fleeson and Wilt's trends, as extraverted behaviour was associated with greater subjective authenticity.

Trying to understand why dispositional introverts and dispositional extraverts report more subjective authenticity when acting extraverted, compared to acting introverted, is complex. More specifically, the condition by disposition interaction is complicated by two factors: the personality differences in how much participants followed instructions in this study (i.e., personality differences in extraverted behaviour), and the direction of and the causal link between positive affect and authenticity. First, the association of authenticity and trait extraversion, within the acting extraverted condition, is complicated by the degree of extraverted behaviour. That is, trait extraverts behaved more extraverted than trait introverts, and thus more extraverted behaviour cooccurs with more subjective authenticity; momentary extraverted behaviour may be causing the authenticity. Alternatively, the greater subjective authenticity for trait extraverts, relative to trait introverts, may be due to disposition-condition agreement; trait extraverts are arguably behaving more authentically when acting extraverted.

Second, positive affect and authenticity are highly correlated (Schlegel et al., 2009) and, more recently, authenticity has been found to fluctuate due to mood. Lenton, Bruder, Slabu and Sedikides (2013) manipulated mood and found that participants 
reported feeling more authentic following a positive mood induction. In my study, participants in the acting extraverted and control conditions reported more positive affect than participants in the acting introverted condition. Thus, the condition differences in authenticity may be a result of the differences in positive affect. Within this study, positive affect and authenticity are correlated at $r=.46, p<.001$ which reduces to $r_{p}$ $=.36, p<.001$ when controlling for condition. There is a large amount of shared variance between authenticity, extraverted behaviour, and positive affect. Unfortunately, the similarity between the acting extraverted and control conditions, across positive affect, authenticity, and extraverted behaviour prevent partitioning the effects of acting instructions, behaviour, and the consequences. Study 2 attempts to address this shortcoming by creating a greater discrepancy in behaviour between the acting extraverted and control condition.

This study also expanded on previous acting studies by exploring effort. Recall that Gallagher et al. (2011) examined perceived effort in a two-condition acting study. They found, for dispositional extraverts, more effort was reported when participants were instructed to act introverted relative to when participants were instructed to acting extraverted. Among dispositional introverts, there was no significant difference in reported effort between participants instructed to act introverted or act extraverted. This study expands on their conclusions by measuring disposition as a continuous measure (versus recruiting extreme groups) and adds a control condition to provide a baseline measure of situational/activity effort. Study 1's findings on effort largely replicate Gallagher et al.'s (2011) results. Not surprisingly, participants in the control condition reported the least effort, compared to both acting conditions. Thus, the instructions to 
monitor, and potentially modify, behaviour required some effort, consistent with the notion of self-regulation. Furthermore, trait extraverts, relative to trait introverts, reported more effort for acting introverted, and trait introverts, relative to trait extraverts, reported more effort for acting extraverted, supporting a counter-dispositional hypothesis: counter-dispositional behaviour is more effortful. Contrastingly, at 1 standard deviation below the mean of trait extraversion (i.e., for dispositional introverts), there are equivalent levels of effort reported between the two acting conditions. This corresponds to Gallagher et al.'s results and is in opposition to a counter-dispositional hypothesis.

This study is the first acting study to include measures of both subjective authenticity and effort. The pattern of results for effort are roughly a mirror image, flipped on the x-axis, of the authenticity findings. Furthermore, the correlation between authenticity and effort was $r=-.71, p<.001$. This suggests that participants perceive ease of acting as being authentic in this context.

Despite the significant condition and condition by disposition interaction for selfreported effort, there were no significant findings for Stroop performance. Actively monitoring and modifying behaviour has been found to result in cognitive fatigue (Hagger et al., 2010). If behaviour was effortful, there should be corresponding trends on Stroop performance. A more detailed account of Stroop performance, and its lack of support for the effort findings, will be returned to later.

Overall, the results from study 1 do not strongly support the negative costs hypothesized to result from the combination of contra-situational and counterdispositional behaviour. Enjoyment and positive affect results suggest that the introverted situation may be causing shifts in the hypothesized directions but these shifts 
were not sufficient to reverse the effects. The dependent variables of positive affect and valence demonstrate the benefit of acting extraverted similar to previous acting research. In contrast, the dependent variables of authenticity and effort suggest that there may be merit in a counter-dispositional interpretation.

One explanation for the mixed findings is that the 'introverted' situation was less than ideal. Despite the comparisons to previous acting studies suggesting that the situation was more introverted, there are indications that the situation was not perfect. First, previous acting studies demonstrate high similarity between the control condition and the acting extraverted condition, for behaviour and affect. Similarly, in this study, the behaviour of participants in the control condition was more comparable to the behaviour of participants in the acting extraverted condition than to participants in the acting introverted condition. Thus in a group of 3, two participants behaved similarly potentially creating the norm for the situation, and this norm was most different from the acting introverted condition. Furthermore, participants in the acting extraverted and control conditions socialized to a greater extent and effectively ignored the participant(s) in the acting introverted condition. Consequences of this group interaction dynamic might leave participants in the acting introverted condition feeling isolated and those in the other conditions feeling more socially accepted. Engaging in social situations has been found to result in pleasant emotions (Clark \& Watson, 1988; Watson, 1988; Watson, Clark, McIntyre, \& Hamaker, 1992). With participants in the acting extraverted and control conditions engaging in more social interaction, there could be affective implications of their behaviour that may be the opposite of what was originally hypothesized. 
Furthermore, the behaviour for participants in the acting extraverted condition could be characterized as more neutral than contra-situational per se. Despite the situation being more introverted than prior acting studies, the average behaviour for the acting extraverted condition was at the midpoint of the scale and it is therefore difficult to determine if it is contra or simply neutral. Supporting the notion that the acting extraverted condition was not contra-normative is its similarity to the control condition. The depleting effects of contra-situational behaviour may not have emerged, as behaviour was not contra despite creating a more introverted situation. A goal for study 2 is to make the acting extraverted condition distinct from the control condition and contra to the situational norm.

Another possibility for the mixed findings is that the greater positive affect reported for acting extraverted was partially due to the choice of adjectives listed in the instructions to modify behaviour. That is, extraverted adjectives (bold, talkative, energetic, active, assertive, and adventurous) are seen as more favourable characteristics relative to introverted adjectives (reserved, quiet, reflective, passive, compliant, and unadventurous). Comparing, where possible ${ }^{18}$, the likability, social desirability (Kirby \& Gardner 1972), and sematic quality (Anderson, 1968) of the adjectives reveals that the extraverted adjectives are rated more positively across all three evaluations compared to the introverted adjectives. Thus, participants may have expectations (good/bad) about

${ }^{18}$ There were ratings for five of the six extraverted adjectives (bold, talkative, energetic, active, and adventurous) across both studies whereas there were only ratings for three of the introverted adjectives. Quiet, passive, and unadventurous were rated on likeability and social desirability and reserved, quiet, and passive were rated on sematic quality. 
how they believe the behaviour would feel, thus potentially influencing positive and pleasant affect results. For study 2, instructions were more balanced, highlighting the negative aspects of acting extraverted and highlighting the positive aspects of acting introverted.

\section{Study 2}

Study 1 found trends that support the idea of counter-dispositional costs, but provided no support for contra-situational costs. The manipulation check showed that the situation was more introverted than previous acting studies, but it may not have been introverted enough; the acting extraverted condition may have been neutral and not contra-situational per se. Furthermore, the acting extraverted and control conditions were highly similar for level of extraverted behaviour, reported positive affect, negative affect, valence, and authenticity. This makes disentangling the implications of situation versus behaviour problematic. For study 2, the situation was constructed to be more introverted than study 1 by having participants' complete jigsaw puzzles individually rather than in groups. Furthermore, participants were provided with a motivational incentive of a small reward to direct behaviour in the intended directions. The acting introverted and control condition participants were informed that they would receive the small reward for completing their jigsaw puzzle within the allotted time, presumably requiring diligent solitary work. Participants in the acting extraverted condition were informed that they would receive the small reward if they prevented the other group members from completing their puzzles through social distraction and chit-chat (i.e., be a mole).

Researchers noted that during study 1 the acting extraverted participants and the control condition participants would interact and effectively seclude the acting 
introverted participant(s) from the social interaction. The differences between conditions for positive affect and valence may be due to participants in the acting introverted condition feeling ostracized. To remove this possible confound, sessions were run in groups of 3 with 1 participant and 2 confederates. Confederates were instructed to keep the situation as consistent as possible across participants/the data collection period and to respond with a neutral expression. The use of confederates eliminates the problem of participants in the acting introverted condition feeling isolated from the situation, allows for greater control of the level of social interaction, and any potential positive social feedback that extraverted behaviour may produce from other participants is also eliminated. Confederates were blind to the premise of the study and blind to participants being instructed to modify behaviour. Each confederate-rated participants' behaviour and affect as a manipulation check. Consistent with study 1, participants were randomly assigned to one of three conditions (acting extraverted, acting introverted, and control condition).

Instructions to modify behaviour employed a series of adjectives to describe introverted or extraverted behaviour. Unfortunately, extraverted terms are generally more favourable than introverted terms (Anderson, 1968; Kirby \& Gardner, 1972). It is possible that the greater positive affect for acting extraverted versus acting introverted is partially due to the difference in expectations about the behaviour created by the favourableness of the adjectives. To reduce this potential influence, instructions were modified to highlight the negative characteristics of acting extraverted and highlight the positive characteristics of acting introverted.

The goals of study 2 were to create a more introverted situation, to control the 
social interaction among group members, reduce anticipated differences, and to motivate behaviour to produce larger discrepancies between acting extraverted and control conditions.

Assessment of Situation and Modified Instruction. To determine if the situation was introverted, in general and compared to study 1, a series of comparisons were made. The average level of extraverted behaviour of the control condition, as participants in this condition did not receive instructions to modify behaviour, was compared to the midpoint of the scale and then compared to study 1 (single sample and independent samples t-tests). Similar comparisons were then examined for the acting extraverted condition to determine if participants' behaviour was contra-situational versus neutral. That is, did the change to instructions create a desired behaviour-situation discrepancy for participants in the acting extraverted condition?

An affective forecasting questionnaire asked participants to predict their affect during the activity. The questionnaire was identical between study 1 and study 2 although for study 1 it was administered for a separate line of inquiry. At the time of administration they had been informed that the task is to complete a jigsaw puzzle and they have read the instructions for their condition but they have not interacted with the other participants/confederates. The task was described as completing a jigsaw puzzle as a group for study 1 and as completing a jigsaw puzzle for study 2 (with no indication of solitary or group activity being indicated). Thus the tasks were described very similarly for both studies whereas the instructions to modify behaviour were different. Contrasting the forecasts between the studies, for positive affect, valence, and negative affect, can reveal the implications of these alterations to instructions. Comparing the control 
conditions establishes if there is an impact for including/omitting as a group. Comparing the acting conditions establishes the impact for the modified behavioural instructions (in combination with the as a group wording discrepancy). The desired changes due to instructions were that acting extraverted would be perceived more negatively for study 2 compared to study 1 where acting introverted would be perceived more positively for study 2 compared to study 1.

One final comparison to assess the modified instructions was to explore the terms introverted and extraverted from the self-reported behaviour questionnaire. The terms introverted and extraverted are not explicitly listed in the altered instructions and thus provide a basis for understanding how participants interpreted the instructions.

Hypotheses. All hypotheses remain identical to study 1 with a combination of counter-dispositional and contra-situational behaviour hypothesized to result in the greatest negative consequences.

\section{Methods}

Participants. Consistent with study 1, participants were recruited through Sona for a study on "individual differences in work and play activities." A total of 100 participants completed the study for course credit. Due to failure to complete the online premeasure, excessively poor English language skills, or scheduling issues (only 1 confederate), the sample was reduced to 85 (66 females, 19 males) for analyses.

\section{Materials.}

Questionnaires assessing personality traits, effort, authenticity, and opinions about the activity, were identical to study 1 with Cronbach alpha results of $.89, .72, .90$, and .71 respectively. The Stroop task was identical to study 1 but was executed with SuperLab 
4.5 operating on iMacs (MA200LL) with the Snow Leopard operating system.

Affect. This questionnaire was modified from study 1 to include the accidentally omitted term fearful to align with previous acting studies. No other changes were made to this questionnaire. The affective composites of positive affect, negative affect, selfconsciousness, and valence remained identical to study 1 (including the omission of the word fearful from negative affect composite to allow direct comparison) with Cronbach alpha results of $.78, .74, .83$, and .68 respectively.

Affective Forecasting. This questionnaire was collected in study 1 for a separate line of inquiry and was retained for study 2 to explore how participants perceived the new instructions. Participants rated a series of adjectives, on a 7-point Likert scale $(1=$ Very slightly or not at all to $7=$ Extremely or a lot) on their predictions or forecasts on how they expected to feel during the upcoming task. Predicted positive affect score was the mean of excited, interested, and strong (Cronbach $\alpha=.74$ ), predicted negative affect was the mean of worried, fearful, upset, nervous, and distressed (Cronbach $\alpha=.85$ ), pleasantness was the mean of happy and pleased (Cronbach $\alpha=.40)$.

Behaviour. This questionnaire remained nearly identical to study 1 . The terms extraverted and introverted were added to this questionnaire, for a total of 20 adjectives, to allow direct determination if participants interpreted the new instructions as extraverted or introverted. The composite score of self-reported extraverted behaviour was thus the mean of energetic, assertive, talkative, shy*, bold, quiet*, extraverted, and introverted $^{*}$ with a Cronbach alpha of .90 . (Items with an asterisk indicate reverse scored items.)

A second composite score was created from the adjectives of this questionnaire. 
Self-reported agreeable behaviour was the mean of cooperative, distrustful*, kind, rude*, trustful, unsympathetic*, and warm with a Cronbach alpha of .68 (Items with an asterisk indicate reverse scored items). Agreeable behaviour, and confederate-ratings of agreeable behaviour, was added to explore the implications of the modified instructions for participants in the acting extraverted condition to derail the confederates from completing their puzzles. More specifically, would confederates still perceive the behaviour of participants in the acting extraverted condition positively (i.e., agreeably) despite them trying to cripple their progress?

Confederate observations. Confederates rated how they perceived the behaviour and affect of the participant. For both questionnaires, confederates were instructed to rate each adjective on how well it described the participant's behaviour/affect. Each adjective was rated on a seven-point Likert type scale $(1=$ strongly disagree to $7=$ strongly agree). To help keep confederates blind to the true purpose of the study, the 50 adjectives represented all big-five personality trait domains (agreeable, open, conscientious, neurotic, and extraverted, see Appendix I). Thirteen adjectives assessed extraverted behaviour. The mean of adventurous, assertive, bashful*, bold, energetic, extraverted, introverted*, quiet*, ${ }^{*}$ assive*, reserved*, shy*, talkative, and withdrawn* (Cronbach $\alpha=.97$, items with an asterisk indicate reverse scored items) created the composite extraverted behaviour scale.

A second behavioural composite score was calculated to assess agreeable behaviour. Eight adjectives, agreeable, cooperative, cold*, harsh*, kind, rude*, unsympathetic* , and warm (Cronbach $\alpha=.93$, items with an asterisk indicate reverse scored items) were averaged to create the composite score of agreeable behaviour. 
Confederates' rated the participants' affect with the affect self-report questionnaire with modified instructions (see Appendix J). Positive affect, negative affect, valence, and self-consciousness were calculated in an identical manner as the selfreports (Cronbach $\alpha=.90, .30, .87, .85$, respectively).

Instruction to modify behaviour. The written instructions to modify behaviour were changed from previous acting studies. Adjectives were amended to highlight more negative aspects of extraverted behaviour (chatterbox, loud) and to highlight more positive aspects of introverted behaviour (contemplative, great listener). The instructions were also modified from a list of adjectives to a narrative paragraph. Key terms for acting extraverted were overly social, chatterbox, busy, loud, energized by the environment, blurts out ideas, and highly assertive. Key terms for acting introverted were introspective, contemplative, enjoys solitude, reflective, energized from within, great listener, reserved, highly observant, and comments are deliberate and relevant. The control condition stated that the upcoming activity was to understand how people work when in close proximity to others as this is a common occurrence in everyday life. There was no mention of specific behaviours for this condition (see Appendix K).

To create greater discrepancies between the acting conditions, all three conditions were eligible to win a small reward. To motivate behaviour in desired directions, the acting introverted and control conditions instructions stated that they must complete their jigsaw puzzle within the allotted time to win the prize whereas, the acting extraverted condition, was informed that to win the prize, they must prevent the other group members from finishing their puzzles. The acting extraverted instructions also stated "we are asking you to be a mole and to distract the other participant(s) with social chit-chat such 
that they do not complete their puzzle".

Situation. Similar to study 1 , the activity took place in a conference room located in the CUHL lab space and directly across the hall from a series of small rooms. The blinds were drawn so that consequences of weather and seasonal views would be minimized. The conference room contained a grouping of tables forming a small conference table layout. This table had three unassembled jigsaw puzzles on it. The puzzles were located in different areas of the table (one on each side and one at the head of the table). Photos of the puzzle image were beside the puzzle pieces and a chair was directly in front of each puzzle.

Role of Confederates. Confederates were kept blind to the true nature of the study and were not aware that participants were assigned to acting conditions. They had three primary goals: to be consistent, have a neutral response, and control the amount of talking during the activity. Confederates were asked to be consistent from session to session in relation to topics discussed, their personal style, how much of the puzzle they completed, and how agreeable/disagreeable they behaved. They were informed that participants completed a measure of emotions immediately after the puzzle activity and to prevent influencing these results that a neutral response was ideal. The most critical instruction, and purpose for having two confederates per testing session, was to control the situation. Confederates would initiate conversation if there had been no talking during the first few minutes. When the participant was talkative, they would also limit responses (avoid eye-contact, restrict responses to yes/no, mumble a non-response, etc.).

Confederates played the role of a participant throughout each session. This included knocking at the door and saying "Hi. I'm here for a study" when arriving. They 
passed papers to the researcher to mimic completion of the informed consent and first questionnaire. Sound files of a Stroop task being completed were played while the participant completed their Stroop task ${ }^{19}$.

\section{Procedure.}

Similar to study 1, this was a two-part study. Part one was the on-line survey and part two was the experimental session conducted in the laboratory setting. The on-line survey was identical to study 1 and contained the questionnaire for disposition and other questionnaires for separate lines of inquiry. Once participants signed-up for the experimental session, they were emailed the invitation code required to access the on-line study. When needed, reminder emails to complete the on-line questionnaire were sent the day prior to their scheduled lab session.

Experimental sessions were run in mock groups with 3 members (1 participant and 2 confederates). This ensured situational consistency with previous research, which has primarily employed three person interactions. Participants were randomly assigned (roll of a die) to one of three conditions (act extraverted, act introverted, or control condition).

Upon arrival at the CUHL lab, the participant was lead to the first room of a series. The room was equipped with a computer desk, computer terminal, and chair. When confederates arrived at the lab they played the role of a participant, including knocking at the door and saying, "Hi. I'm here for a study." In some instances, both confederates

${ }^{19}$ In piloting this design, two participants explicitly asked why the other participants were not completing the Stroop task. To prevent participants from understanding the true nature of the other participants, sound files of the Stroop task being completed were always played. 
arrived before the participant. In other sessions, they arrived after the participant or a mix of one before and one after (depending partially on the time of arrival of the participant). That is, when the participant was on time or late, the other group members were already in the lab whereas when the participant was early, confederates would sneak out of the lab and arrive a few minutes later. Confederates were located in the remaining two rooms in the series.

Once all group members had arrived, the experimental session began with a verbal orientation to the study and completing the informed consent. Participants were then given the instruction sheet, which contained condition information and the affective forecasting questionnaire. Once the questionnaire was completed, the mock group was informed that they were moving to the larger room to complete jigsaw puzzles. The researcher directed each group member to a specific puzzle. The participant always completed the same puzzle and sat in the same location at the conference table. Once everyone was seated at the conference table, the group was reminded that they had a total of 25 minutes for the activity, all pieces of the puzzle were there, and then they were instructed to begin.

After 15 minutes elapsed, the group was informed that they would move back to their original rooms to complete a questionnaire and a computer task before returning for the remaining 10 minutes of the puzzle activity. Once group members were back in their cubicles, participants completed the emotions, behaviour, authenticity, effort, and enjoyment of activity questionnaires and confederates completed the confederate observation (behaviour and affect) questionnaire. Participants then completed the Stroop task following verbal and visually presented instructions. Participants were told to go as 
quickly and accurately as possible. The computers in the confederates' rooms played a sound file of a Stroop task being completed (keys being hit on the response pad). The Stroop task ends with instructions on the screen informing the participant that the task is complete and to get the researcher. Participants were then informed that they did not need to continue with the puzzle task and were then debriefed about the true nature of the study and the role of the confederates. All participants received the small prize of a bag of candy. Due to the deception involved, a second informed consent (permission to use data) was also dispensed. Finally, participants were thanked for their time.

\section{Preliminary Data Exploration}

To explore the relationships between the different dependent variables, bivariate correlations were computed. Table 6 displays the correlations between the ten selfreported dependent variables, Table 7 displays the correlations between the seven confederate-rated dependent variables and Table 8 displays the correlations between the ten self-rated and seven confederate-rated dependent variables. For both self-rated and confederate-rated dependent variables, positive affect was significantly correlated with extraverted behaviour, valence, and arousal; negative affect was significantly positively correlated with self-consciousness and negatively correlated with valence.

For the self-reported dependent variables, extraverted behaviour was also significantly positively correlated with effort. Of note was extraverted behaviour's lack of significant correlation with valence, self-consciousness, authenticity, or enjoyment. The lack of association between extraverted behaviour and both authenticity and enjoyment are novel to this study. Agreeable behaviour was significantly positively correlated with valence and self-consciousness. Authenticity was positively correlated 
with agreeable behaviour and enjoyment and negatively correlated with selfconsciousness and effort. The strong negative association between authenticity and effort may be partially due to the items of the scales presented on the same questionnaire page.

For the confederate-rated dependent variables, all correlations between the variables were significant. The magnitude of the correlations ranged from -.22 (positive and negative affect) to .92 (positive affect and arousal). Two correlations were exceptionally high $(r>.90)$ indicating a lack of distinctiveness between the variables. Both of these correlations include the variable of arousal and its associations with positive affect and extraverted behaviour. It is also noteworthy that positive affect and extraverted behaviour were highly correlated $(r=.82)$. The correlations between selfrated and confederate-rated matched dependent variables produced mixed results. Importantly, there was a strong correlation between self-reported and confederate-rated extraverted behaviour indicating good agreement. There were also positive correlations between ratings for positive affect and arousal whereas the ratings of agreeable behaviour, negative affect and self-consciousness were not correlated. Lack of agreement between confederate-rated and self-rated behaviour and emotion variables, in combination with strong correlations among all the confederate-rated variables, may indicate a problem with confederate ratings, a point that will be returned to later. 
Table 6

Correlations Between Self-Reported Behaviour and Affect for Study 2

\begin{tabular}{|c|c|c|c|c|c|c|c|c|c|}
\hline \multicolumn{10}{|l|}{ Self-Ratings } \\
\hline & 1 & 2 & 3 & 4 & 5 & 6 & 7 & 8 & 9 \\
\hline 1. Extraverted Behaviour & - & & & & & & & & \\
\hline 2. Agreeable Behaviour & .19 & - & & & & & & & \\
\hline 3. Positive Affect & $.30 * *$ & .13 & - & & & & & & \\
\hline 4. Negative Affect & .11 & -.18 & .00 & - & & & & & \\
\hline 5. Valance & .11 & $.38 * * *$ & $.62 * * *$ & $-.51 * * *$ & - & & & & \\
\hline 6. Arousal & $.53 * * *$ & .14 & $.72 * * *$ & -.04 & $.41 * * *$ & - & & & \\
\hline 7. Self-consciousness & .16 & $-.33 * *$ & -.04 & $.83 * * *$ & $-.55 * * *$ & .06 & - & & \\
\hline 8. Enjoyment & -.18 & .14 & $.54 * * *$ & $-.19 *$ & $.55^{* * *}$ & $.22 *$ & $-.33 * *$ & - & \\
\hline 9. Authenticity & -.12 & $.33 * *$ & .08 & -.06 & .17 & -.13 & $-.26^{*}$ & $.38 * * *$ & - \\
\hline 10. Effort & $.33 * *$ & -.20 & -.02 & $.33 * *$ & $-.27^{*}$ & .09 & $.43 * * *$ & $-.50 * * *$ & $-.70 * * *$ \\
\hline
\end{tabular}

Note: $* p<.05, * * p<.01, * * * p<.001$ 
Table 7

Correlations Between Confederate-Rated Behaviour and Affect for Study 2

\begin{tabular}{|c|c|c|c|c|c|c|}
\hline \multicolumn{7}{|l|}{ Confederate Ratings } \\
\hline 1. Extraverted Behaviour & - & & & & & \\
\hline 2. Agreeable Behaviour & $.58 * * *$ & 一 & & & & \\
\hline 3. Positive Affect & $.82 * * *$ & $.63 * * *$ & - & & & \\
\hline 4. Negative Affect & $-.33 * *$ & $-.44 * * *$ & $-.22 *$ & - & & \\
\hline 5. Valence & $.61 * * *$ & $.70 * * *$ & $.71 * * *$ & $-.58 * * *$ & - & \\
\hline 6. Arousal & $.91 * * *$ & $.66 * * *$ & $.92 * * *$ & $-.30 * *$ & $.66^{* * *}$ & - \\
\hline 7. Self-consciousness & $-.58 * * *$ & $-.32 * *$ & $-.52 * * *$ & $.60 * * *$ & $-.55 * * *$ & $-.52 * * *$ \\
\hline
\end{tabular}

Note: ${ }^{*} p<.05, * * p<.01, * * * p<.001$ 
Table 8

Correlations Between Self-Reported Behaviour and Affect to Confederate-Rated Dependent Variables for Study 2

\begin{tabular}{|c|c|c|c|c|c|c|c|}
\hline \multirow[t]{2}{*}{ Self-Ratings } & \multicolumn{6}{|c|}{ Confederate Ratings } & \multirow[b]{2}{*}{7} \\
\hline & 1 & 2 & 3 & 4 & 5 & 6 & \\
\hline 1. Extraverted Behaviour & $.71 * * *$ & $.40 * * *$ & $.53 * * *$ & $-.28 * *$ & $.49 * * *$ & $.56^{* * *}$ & $-.29 * *$ \\
\hline 2. Agreeable Behaviour & .09 & .11 & .07 & -.14 & $.22 *$ & .03 & -.21 \\
\hline 3. Positive Affect & $.25 *$ & .14 & $.37 * * *$ & -.08 & $.35 * * *$ & $.30 * *$ & -.19 \\
\hline 4. Negative Affect & .09 & .01 & .01 & .09 & -.05 & .05 & .09 \\
\hline 5. Valance & .14 & $.15^{*}$ & $.22 *$ & -.18 & $.32 * *$ & .16 & $-.23 *$ \\
\hline 6. Arousal & $.52 * * *$ & $.33 * *$ & $.51 * * *$ & $-.24 *$ & $.40 * * *$ & $.53 * * *$ & $-.36 * * *$ \\
\hline 7. Self-consciousness & .16 & .00 & .04 & .10 & -.12 & .13 & .09 \\
\hline 8. Enjoyment & -.19 & -.10 & -.04 & .03 & .01 & -.11 & .02 \\
\hline 9. Authenticity & $-.34 * *$ & -.11 & -.21 & .05 & .01 & $-.30 * *$ & .17 \\
\hline 10. Effort & $.38 * * *$ & .09 & .21 & -.01 & .04 & $.29 * *$ & -.12 \\
\hline
\end{tabular}

Note: ${ }^{*} p<.05, * * p<.01, * * * p<.001$, Self-observer matched correlations are bolded. 


\section{Manipulation Check.}

A manipulation check was conducted to determine if participants followed acting instructions and to explore the potential influence of disposition on adherence to instructions. A three-step hierarchical regression analysis was conducted on extraverted behaviour with condition (dummy coded) entered first. Then disposition (centred) and two interaction terms were added in distinct steps. There was a condition effect for selfreported extraverted behaviour, $R^{2}=.403, F(2,82)=27.71, p<.001$, indicating at least one condition differed from another. Post-hoc comparisons revealed that participants in the acting extraverted condition $(M=4.97)$ reported acting more extraverted compared to participants in both the control $(M=3.39)$ and acting introverted conditions $(M=3.26)$, which did not significantly differ. Thus, it appears that participants followed instructions to modify behaviour. There was a significant general trend of disposition, $b=.32, \Delta R^{2}$ $=.031, p=.04$, indicating that as trait extraversion increases, reported extraverted behaviour also increased across conditions. The interaction between condition and disposition was non-significant, $\Delta R^{2}=.025, \Delta F(2,79)=1.85, p=.16$.

Confederates rated participants' levels of extraverted behaviour and thus provide a second form of manipulation check. In addition, confederates were blind to the premise of the study and thus provide an unbiased perception of extraverted behaviour. Confederate ratings of participants' extraverted behaviour show a significant effect of condition, $R^{2}=.632, F(2,82)=70.42, p<.001$, indicating that at least one condition differed from another. Post-hoc comparisons revealed that participants in the acting extraverted condition were seen as behaving more extraverted $(M=5.78)$ compared to participants in the control $(\mathrm{M}=3.69)$ and acting introverted conditions $(M=3.26)$, which 
did not differ. Targets' disposition was related to how participants were perceived, $b$ $=.35, \Delta R^{2}=.037, p=.004$, across conditions, trait extraverts were perceived as behaving more extraverted than trait introverts. The interaction between targets' disposition and condition was non-significant for confederate-rated extraverted behaviour, $\Delta R^{2}=.009$, $\Delta F(2,79)=1.10, p=.34$. Self-reported extraverted behaviour is fully corroborated by confederate-rated level of extraverted behaviour. Participants in the acting extraverted condition behaved more extraverted than the other conditions. Thus, it appears that the instructions to modify behaviour generally worked despite the similarity between control and acting introverted.

Based on the similarity of acting extraverted and control condition scores of previous studies, on multiple dependent variables, a key goal for study 2 was to dissociate the level of extraverted behaviour between participants in the acting extraverted and control conditions. As noted above, the post-hoc comparisons for the condition effect of extraverted behaviour revealed that participants in the acting extraverted condition reported and were perceived as behaving more extraverted than participants in the control condition, $t(53)=5.37, p<.001$, Cohen's $d=1.45 ; t(53)=8.84, p<.001$, Cohen's $d=$ 2.38 for self-reported and confederate-perceived respectively. Thus, the objective of making these two conditions distinct was achieved.

Ideally, the behaviour for participants in the acting introverted and control conditions would have differed from each other. Unfortunately, these conditions were not significantly different although the direction of difference was in the intended direction with participants in the acting introverted condition reporting less extraverted behaviour than participants in the control condition, $t(55)=-1.94, p=.06$, Cohen's $\mathrm{d}$ 
$=.52^{20}$

\section{Assessment of Situation and Modified Instructions}

Across both studies, the primary goal was to create a normatively introverted

situation in the lab. The control condition, where there were no instructions to modify behaviour, provided a baseline for determining the normative behaviour of the situation.

For study 2 , the control condition mean $(M=3.39)$ was slightly introverted on the 7-point Likert scale. Comparing the control condition mean to the midpoint of the scale reveals a moderate effect, $t(26)=-4.02, \mathrm{p}<.001$, Cohen's $d=.77$, indicating that the situation is moderately introverted. Relative to study 1 , the introverted situation effect size of study 2 is stronger (study 1 control condition to midpoint of Likert scale, $t(48)=-1.90, p=.06$, Cohen's $d=.27$ ). An independent samples t-test of control conditions between the two studies was not significant, $t(74)=1.08, p=.28$, Cohen's $d=.26$. Thus, the solitary puzzle activity task created a normatively introverted situation although the introverted situation was not more introverted than study 1 .

To determine if participants in the acting extraverted condition behaved in a contra-situational manner, the average level of extraverted behaviour is compared to the midpoint of the Likert scale and compared to study 1 . For study 2, the average level of extraverted behaviour in the acting extraverted condition is greater than the midpoint on the scale, $t(27)=3.89, p=.001$, Cohen's $d=.73$. Relative to study $1, t(47)=2.54, p$

${ }^{20}$ A sample size of 60 participants per condition would be required for this effect to reach significance given alpha of .05 and power of .80. The lack of significance with a moderate effect size, makes the smaller sample size of study 2 salient. Caution for interpreting similarities and discrepancies between these two conditions should be exercised. 
$=.01$, Cohen's $\mathrm{d}=.37$, the contra-situational effect size is significantly stronger, $t(74)=$ $2.14, p=.04$, Cohen's $d=.47$. For study 2 , the situation was still introverted and participants in the acting extraverted condition behaved more extraverted and thus it can be concluded that participants in the acting extraverted condition behaved more contrasituational compared to study 1 .

To explore the implications of the altered instructions, affective forecasts for positive affect, valence, and negative affect were compared between the studies (see Table 9). Independent t-tests, with a pooled variance term, indicated that there were no affective forecasting differences for the control condition. Acting extraverted was expected to be less pleasant and more negative for study 2 compared to study 1 . Acting introverted was expected to result in more positive affect and pleasantness for study 2 compared to study 1 . Thus, the altered instructions did seem to change participants' expectations about the upcoming behaviours in the intended directions with the acting extraverted expectation being less pleasant and more negative and the acting introverted expectations being more positive and pleasant. 
Table 9

Comparing Forecasted Affect by Condition and by Study

\begin{tabular}{cccccccc}
\hline & \multicolumn{5}{c}{ Study1 } & \multicolumn{7}{c}{ Study 2 } & \multirow{2}{*}{ t-test } \\
\cline { 2 - 7 } & $\mathrm{n}$ & Mean & SD & $\mathrm{n}$ & Mean & SD & \\
\cline { 2 - 7 } Acting Extraverted & & & & & & & \\
$\quad$ Positive Affect & 48 & 4.63 & 1.08 & 28 & 4.33 & 1.18 & 1.16 \\
$\quad$ Valence & 48 & 4.43 & 1.01 & 28 & 3.93 & 0.81 & $2.17^{*}$ \\
$\quad$ Negative Affect & 48 & 2.70 & 1.22 & 28 & 3.53 & 1.28 & $-3.11^{* *}$ \\
Control & & & & & & & \\
$\quad$ Positive Affect & 49 & 4.42 & 1.04 & 26 & 4.68 & 0.70 & -1.10 \\
$\quad$ Valence & 49 & 4.57 & 0.97 & 26 & 4.48 & 0.94 & 0.38 \\
$\quad$ Negative Affect & 49 & 2.23 & 0.89 & 26 & 2.37 & 0.89 & 0.60 \\
Acting Introverted & & & & & & & \\
$\quad$ Positive Affect & 60 & 3.54 & 1.48 & 30 & 4.23 & 1.11 & $-2.64^{* *}$ \\
Valence & 60 & 3.78 & 1.23 & 30 & 4.27 & 0.91 & $-2.04^{*}$ \\
Negative Affect & 60 & 2.28 & 0.96 & 30 & 2.73 & 1.18 & -1.97 \\
\hline
\end{tabular}

Note: $* \mathrm{p}<.05, * * \mathrm{p}<.01$

Study 2 added the terms introverted and extraverted to the behaviour questionnaire. As the instructions to manipulate behaviour do not explicitly use these terms and describe introverted and extraverted behaviour, these terms are ideal for exploring if participants interpreted the instructions as desired. Analysis of Variance tests were conducted to explore condition differences. Where appropriate, significant omnibus tests were followed up with post-hoc comparisons with Tukey Honestly Significant Difference. For both items, there was a significant condition effect, $F(2,82)=$ $14.61, p<.001 ; F(2,81)=23.85, p<.001$ for items extraverted and introverted respectively. Participants in the acting extraverted condition reported behaving more extraverted and less introverted than the other two conditions, which did not differ (see Table 10). Therefore, despite not explicitly using the terms introverted or extraverted in the instructions to manipulate behaviour, participants understood the notion of behaving 
EXTRAVERSION AND COUNTER-DISPOSITIONAL BEHAVIOUR

extraverted or introverted.

Table 10

Means by Condition for the Behaviour Items of Introverted and Extraverted

\begin{tabular}{llll}
\hline Behaviour Item & $\begin{array}{l}\text { Acting } \\
\text { Extraverted }\end{array}$ & Control & $\begin{array}{l}\text { Acting } \\
\text { Introverted }\end{array}$ \\
\hline Extraverted & $4.79_{\mathrm{a}}$ & $3.04_{\mathrm{b}}$ & $2.73_{\mathrm{b}}$ \\
Introverted & $2.37_{\mathrm{a}}$ & $4.26_{\mathrm{b}}$ & $5.10_{\mathrm{b}}$ \\
\hline
\end{tabular}

Note: differing subscripts indicate mean differences at least at the $p<.05$ level.

Overall, like study 1, study 2 created a situation that was introverted. Although study 2 was not statistically significantly more introverted than study 1 , the effect size due to the situation was larger. Manipulation checks indicate that participants followed instructions. Although the similarity of behaviour between control condition and acting introverted was less than ideal, participants' behaviour in the acting extraverted condition is more contra-situational compared to study 1 . The affective forecasting results indicate the new instructions changed participants' expectations about the upcoming behaviours. Despite participants in the acting extraverted condition expecting less pleasantness and more negative affect, they were still able to follow instructions and even behaved more extraverted than in study 1 . In combination, the introverted situation with more extraverted behaviour, allows me to conclude that participants in the acting extraverted condition behaved in a more contra-situational manner compared to study 1 .

\section{Results}

For each dependent variable, a three-step hierarchical regression analysis was conducted such that step 1 of the regression contained the two acting condition variables (dummy coded), step 2 added disposition (centred), and finally step 3 added the 
interaction terms ( 2 variables created by multiplying the centered disposition by dummy coded condition variable).

Positive Affect. Motivated by situational self-regulation and previous studies on counter-dispositional behaviour, I hypothesized a condition effect with participants in the acting extraverted condition reporting the least positive affect. I also hypothesized an interaction between disposition and condition with the acting extraverted condition having a positive simple slope and not the other conditions. That is, I expected acting extraverted to be least enjoyable for dispositional introverts. For positive affect, the condition effect, $R^{2}=.041, F(2,82)=1.76, p=.18$, disposition effect, $\Delta R^{2}=.004, b=.10$, $p>.50$, and the condition by disposition interaction, $\Delta R^{2}=.016, \Delta F(2,79)=0.66, p>.50$, were all non-significant. Thus, results do not show either a contra-situational or counterdispositional trends, and support for hypotheses was not found. Participants reported similar levels of positive affect across conditions (see Table 11 for means by condition) and disposition, a notable departure from study 1 and other acting research.

Confederate ratings of positive affect showed a significant condition effect, $R^{2}=$ $.397, F(2,82)=26.98, p<.001$. Post-hoc comparisons revealed that participants in the acting extraverted condition were perceived as experiencing more positive affect than participants in the other two conditions (which did not differ). Targets' disposition, $\Delta R^{2}$ $=.009, b=.13, p=.27$, and the condition by disposition interaction, $\Delta R^{2}=.014$, $\Delta F(2,79)=0.94, p=.40$, were both non-significant.

Valence. I hypothesized a condition effect with participants in the acting extraverted condition reporting the least positive valence. I also hypothesized an interaction between disposition and condition with the acting extraverted condition 
having a positive simple slope (introverts reporting less positive valence than extraverts).

For valence, condition was non-significant, $R^{2}=.024, F(2,82)=1.02, p=.37$, while disposition was marginally significant, $\Delta R^{2}=.042, b=.26, p=.06$, indicating that as trait extraversion increases, more happiness was reported. The condition by disposition interaction was non-significant, $\Delta R^{2}=.023, \Delta F(2,79)=0.99, p=.38$. Thus, the hypothesized condition effect and disposition by condition interaction for valance were not found.

Confederate-rated valence showed a significant condition effect, $R^{2}=.155$, $F(2,82)=7.50, p=.001$; post-hoc comparisons revealing that acting extraverted was perceived as experiencing more positive valence than both the other conditions (which did not differ). There was no significant difference in perceived valence due to targets' disposition, $\Delta R^{2}=.002, b=.06, p>.50$, or the interaction of condition by disposition, $\Delta R^{2}=.001, \Delta F(2,79)=0.03, p>.50$.

Enjoyment. I hypothesized an interaction between disposition and condition with the simple slope for acting extraverted being positive and the simple slope of acting introverted being negative (control was expected to be flat). There were significant condition differences, $R^{2}=.081, F(2,82)=3.63, p=.03$, with participants in the control condition reporting the most enjoyment and participants in the acting extraverted condition reporting the least (the acting introverted condition did not differ from the other two conditions). This is a notable departure from study 1 and other acting research where acting extraverted has been the most enjoyable condition. Disposition and the condition by disposition interaction were non-significant, $\Delta R^{2}=.017, b=.22, p=.23$; and $\Delta R^{2}$ $=.002, \Delta F(2,79)=0.11, p>.50$ respectively. The hypothesized disposition by condition 
interaction for enjoyment was not found.

Negative Affect. Drawing on the idea that counter-dispositional and contrasituational behaviour produces negative outcomes, I hypothesized an interaction of condition by disposition with the acting extraverted simple slope being negative. That is, dispositional introverts in the acting extraverted condition were expected to experience the most negative affect. The condition effect was non-significant, $R^{2}=.026, F(2,82)=$ $1.11, p<.34$, indicating that participants reported similar levels of negative affect across the conditions. There is a significant dispositional effect, $\Delta R^{2}=.056, b=-.30, p=.03$, indicating that as trait extraversion increased, less negative affect was reported. The interaction between condition and disposition was non-significant, $\Delta R^{2}=.033, \Delta F(2,79)$ $=1.47, p=.24$. Thus, the hypothesized disposition by condition interaction for negative affect was not found. For confederate-rated negative affect, all effects were nonsignificant, condition, $R^{2}=.034, F(2,82)=1.47, p=.24$; targets' disposition, $\Delta R^{2}=.002$, $b=-.02, p>.50 ;$ and interaction, $\Delta R^{2}=.008, \Delta F(2,79)=0.33, p=.72$.

Self-consciousness. I hypothesized a condition by disposition interaction with introverted participants reporting more self-consciousness in the acting extraverted condition. There was a marginal effect of condition, $R^{2}=.055, F(2,82)=2.40, p=.10$, with participants in the control condition reporting the least self-consciousness and participants in the acting extraverted condition reporting the most. There was a significant disposition effect, $\Delta R^{2}=.069, b=-.49, p=.01$, indicating that as trait extraversion increased, less self-consciousness was reported. The interaction between condition and disposition was non-significant, $\Delta R^{2}=.023, \Delta F(2,79)=1.05, p=.36$. The hypothesized condition by disposition interaction was not found. 
Confederate-rated self-consciousness revealed a significant condition effect, $R^{2}$ $=.083, F(2,82)=3.72, p=.03$, with participants in the acting extraverted condition perceived as experiencing the least self-consciousness and participants in the acting introverted condition perceived as experiencing the most (neither differed from control). The targets' disposition, $\Delta R^{2}=.011, b=-.12, p=.32$, and the condition by targets' disposition interaction were non-significant, $\Delta R^{2}=.022, \Delta F(2,79)=0.99, p=.38$. Confederate-rated self-consciousness did not completely agree with self-reported selfconsciousness.

Arousal. As with study 1, an exploratory analysis was conducted on arousal, there was a significant condition effect, $R^{2}=.15, F(2,82)=7.17, p=.001$, with the acting conditions being distinct from each other (but neither differing from the control condition). Participants in the acting extraverted condition reported more arousal than participants in the acting introverted condition. There was no significant disposition effect, $\Delta R^{2}=.008, b=.11, p=.38$, and the interaction of condition by disposition was also non-significant, $\Delta R^{2}=.000, \Delta F(2,79)=0.01, p=.99$.

Confederate-rated arousal revealed a significant condition effect, $R^{2}=.485$, $F(2,82)=38.61, p<.001$, with participants in the acting extraverted condition being perceived as more aroused than participants in the other two conditions (which did not differ). There was a marginal disposition trend, $\Delta R^{2}=.020, b=.19, p=.07$, indicating that as the targets' trait extraversion increased, more arousal was perceived. The interaction between condition and targets' disposition was non-significant, $\Delta R^{2}=.007$, $\Delta F(2,79)=0.54, p>.59$

Authenticity. I hypothesized a condition effect with participants in the control 
condition reporting the most authenticity and participants in the acting extraverted condition the least. I also expected an interaction between disposition and condition with introverts in the acting introverted condition reporting more authenticity than extraverts and extraverts in the acting extraverted condition reporting more authenticity than introverts. There was a significant condition effect, $R^{2}=.134, F(2,81)=6.29, p=.003$, with participants in the control condition reporting the most authenticity and participants in the acting extraverted condition reporting the least (neither of these condition differed from acting introverted). Disposition and the condition by disposition interaction were non-significant, $\Delta R^{2}<.001, b=.02, p>.50$ and $\Delta R^{2}=.017, \Delta F(2,78)=0.76, p=.47$, respectively. Results support the hypothesized condition effect but there is no evidence for the hypothesized interaction.

Effort. I hypothesized a condition effect with participants in the acting extraverted condition reporting more effort than the other conditions. I also expected that trait introverts in the acting extraverted condition would report more effort than extraverted participants (an interaction with a positive simple slope for acting extraverted). There were a significant condition differences, $R^{2}=.122, F(2,81)=5.64, p$ $=.005$, with participants in the acting extraverted condition reporting the most effort compared to the other two conditions (which did not differ). Disposition and the condition by disposition interaction were both non-significant, $\Delta R^{2}=.002, b=-.08, p$ $>.50$ and $\Delta R^{2}=.001, \Delta F(2,79)=0.06, p>.50$, respectively. Results support the hypothesized condition effect but there was no evidence for the hypothesized interaction.

Cognitive Fatigue. I hypothesized that dispositional introverts, in the acting extraverted condition, would perform worse than dispositional extraverts in that condition. 
I did not posit performance differences due to disposition for the other two conditions. Similar to study 1, Stroop performance was examined in multiple ways. Errors ranged from zero to 40 for untrimmed $(M=7.49, S D=7.43)$ and from zero to 39 for trimmed data $(M=6.13, S D=6.19)$. Across all Stroop performance dependent variables, findings were non-significant with no p-value approaching significance (see Table 12). Thus, there were no measurable cognitive fatigue effects found for counter-dispositional or contra-situational behaviour and the hypothesized interaction was not found. 
Table 11

Mean Differences by Condition For Each Dependent Variable for Study 2

\begin{tabular}{llll}
\hline Dependant Variable & $\begin{array}{l}\text { Acting } \\
\text { Extraverted }\end{array}$ & Control & $\begin{array}{l}\text { Acting } \\
\text { Introverted }\end{array}$ \\
\hline Extraverted Behaviour & $4.97_{\mathrm{a}}$ & $3.39_{\mathrm{b}}$ & $2.88_{\mathrm{b}}$ \\
Observer Extraverted Behaviour & $5.78_{\mathrm{a}}$ & $3.69_{\mathrm{b}}$ & $3.26_{\mathrm{b}}$ \\
Positive Affect & $4.71_{\mathrm{a}}$ & $4.73_{\mathrm{a}}$ & $4.25_{\mathrm{a}}$ \\
Observer Positive Affect & $5.31_{\mathrm{a}}$ & $4.10_{\mathrm{b}}$ & $3.75_{\mathrm{b}}$ \\
Negative Affect & $3.18_{\mathrm{a}}$ & $2.78_{\mathrm{a}}$ & $2.99_{\mathrm{a}}$ \\
Observer Negative Affect & $2.13_{\mathrm{a}}$ & $2.24_{\mathrm{a}}$ & $2.33_{\mathrm{a}}$ \\
Valence & $5.30_{\mathrm{a}}$ & $5.41_{\mathrm{a}}$ & $5.05_{\mathrm{a}}$ \\
Observer Valence & $5.95_{\mathrm{a}}$ & $5.30_{\mathrm{b}}$ & $5.07_{\mathrm{b}}$ \\
Arousal & $5.05_{\mathrm{a}}$ & $4.61_{\mathrm{ab}}$ & $4.21_{\mathrm{b}}$ \\
Observer Arousal & $5.68_{\mathrm{a}}$ & $4.31_{\mathrm{b}}$ & $4.06_{\mathrm{b}}$ \\
Self-Consciousness & $3.75_{\mathrm{a}}$ & $2.93_{\mathrm{a}}$ & $3.26_{\mathrm{a}}$ \\
Observer Self-Consciousness & $1.89_{\mathrm{a}}$ & $2.29_{\mathrm{ab}}$ & $2.49_{\mathrm{b}}$ \\
Authenticity & $3.48_{\mathrm{a}}$ & $4.76_{\mathrm{b}}$ & $3.99_{\mathrm{b}}$ \\
Effort & $3.71_{\mathrm{a}}$ & $2.65_{\mathrm{b}}$ & $2.91_{\mathrm{b}}$ \\
Enjoyment & $4.87_{\mathrm{a}}$ & $5.80_{\mathrm{b}}$ & $5.42_{\mathrm{ab}}$ \\
Stroop Errors & $7.32_{\mathrm{a}}$ & $5.78_{\mathrm{a}}$ & $5.33_{\mathrm{a}}$ \\
Stroop Response Time & $949.80_{\mathrm{a}}$ & $934.72_{\mathrm{a}}$ & $973.08_{\mathrm{a}}$ \\
Stroop RT Correct & $947.72_{\mathrm{a}}$ & $934.35_{\mathrm{a}}$ & $973.72_{\mathrm{a}}$ \\
Stroop Median RT & $887.98_{\mathrm{a}}$ & $864.22_{\mathrm{a}}$ & $898.65_{\mathrm{a}}$ \\
Stroop Interference RT & $59.20_{\mathrm{a}}$ & $53.94_{\mathrm{a}}$ & $65.46_{\mathrm{a}}$ \\
\hline Note: difering & $5 \mathrm{a}$ & & \\
\hline
\end{tabular}

Note: differing subscripts indicate mean differences at least at the $\mathrm{p}<.05$ level. 
EXTRAVERSION AND COUNTER-DISPOSITIONAL BEHAVIOUR

Table 12

Hierarchical Regression Results for Condition Effect, Disposition Effect and Their

Interaction Across Dependent Variables Assessing Cognitive Fatigue for Study 2

\begin{tabular}{llll}
\hline $\begin{array}{l}\text { Dependent } \\
\text { Variable }\end{array}$ & Condition effect & Disposition effect & $\begin{array}{l}\text { Condition by } \\
\text { Disposition } \\
\text { (Interaction) }\end{array}$ \\
\hline Errors & $R^{2}=.015, F(2,82)=$ & $\Delta R^{2}=.000$, & $\Delta R^{2}=.002, \Delta F(2,79)=$ \\
& $.64, p>.50$ & $b=-.04, p>.50$ & $0.08, p>.50$ \\
Mean RT & $R^{2}=.012, F(2,82)=$ & $\Delta R^{2}=.000$, & $\Delta R^{2}=.012, \Delta F(2,79)=$ \\
& $0.50, p>.50$ & $b=2.52, p>.50$ & $0.50, p>.50$ \\
Mean & $R^{2}=.013, F(2,82)=$ & $\Delta R^{2}=.000$, & $\Delta R^{2}=.013, \Delta F(2,79)=$ \\
Correct RT & $0.54, p>.50$ & $b=2.54, p>.50$ & $0.51, p>.50$ \\
Median RT & $R^{2}=.014, F(2,82)=$ & $\Delta R^{2}=.000$, & $\Delta R^{2}=.015, \Delta F(2,79)=$ \\
& $0.59, p>.50$ & $b=-1.39, p>.50$ & $0.61, p>.50$ \\
Interference & $R^{2}=.009, F(2,82)=$ & $\Delta R^{2}=.010$, & $\Delta R^{2}=.008, \Delta F(2,79)=$ \\
RT & $.37, p>.50$ & $b=6.48, p>.50$ & $0.31, p>.50$ \\
\hline
\end{tabular}

Agreeable Behaviour. An exploratory analysis was conducted on agreeable

behaviour. There were no condition differences, $R^{2}=.007, F(2,82)=.28, p>.50$,

although there was a significant disposition effect, $\Delta R^{2}=.052, b=.26, p=.04$ indicating that as dispositional extraversion increases, more agreeable behaviour was reported. The interaction of condition by disposition was non-significant for self-reported agreeable behaviour, $\Delta R^{2}=.008, \Delta F(2,79)=0.35, p>.50$.

For confederate-rated agreeable behaviour, there was a condition effect, $R^{2}=.158$, $F(2,82)=7.72, p>.001$. Post-hoc comparisons reveal that participants in the acting extraverted condition were perceived as behaving significantly more agreeable that the other two conditions (which did not differ). Targets' disposition and the condition by targets' disposition interaction were both non-significant, $\Delta R^{2}=.003, b=.06, p>.50$; $\Delta R^{2}=.011, \Delta F(2,79)=0.35, p>.50$, respectively. 


\section{Discussion}

This study sought to intensify the contra-situational attributes of extraverted behaviour (cf. study 1) by creating a more introverted situation and motivationally encouraging the desired behaviours. Unfortunately, despite the successful creation of an introverted situation and successfully creating changes in behaviours, I failed to find much evidence for the hypothesized combination of counter-dispositional and contrasituational effects. Despite not finding evidence for the combination of counterdispositional and contra-situational effects (represented by non-significant condition by disposition interactions), there were indications that the situation is important. Unlike previous acting studies, in study 2 , participants in the acting extraverted condition did not report more positive affect and instead reported the least enjoyment compared to participants in the control condition. Furthermore, participants in the acting extraverted condition reported the most amount of effort. Thus, this study found that acting extraverted, in an introverted situation, was associated with less enjoyment and more effort. However, participants in the acting extraverted condition failed to report more negative affect or show effects of cognitive fatigue and reported feeling only marginally more self-conscious. Therefore, this study eliminated the normally found benefits of acting extraverted, greater positive affect and enjoyment, without causing negative consequences, greater negative affect, or cognitive depletion effects. Thus, this study adds the caveat that behaving extraverted may not always be beneficial.

Negative consequences for counter-dispositional behaviour have been limited to extraverts in the acting introverted condition (Gallagher et al., 2011; Zelenski et al., 2012). One possible justification for why counter-dispositional effects have not been 
previously found for introverts is that the positive affect experienced in the acting extraverted condition buffers, or concurrently restores, any depleting effects. By removing the high levels of positive affect associated with acting extraverted, compared to acting introverted, the results of study 2 provide a new piece of information against the buffering explanation. More specifically, I removed the positive impact of acting extraverted and still did not find evidence for cognitive fatigue or negative affect for introverts. Thus, this study provides evidence that positive affect is not essential for buffering cognitive fatigue effects for introverts in the acting extraverted condition.

Participants reported feeling most authentic about their behaviour when not given instructions to modify behaviour. As expected, acting extraverted was the least authentic and most effortful condition. Given that the situation was introverted, this lends support to the notion that contra-situational behaviour requires self-regulation (although caution should be exercised with this claim as there was no evidence for cognitive fatigue effects). Thus, these trends suggest that the situation may be important. Moreover, across conditions, participants reported the least authenticity in the acting extraverted condition. This finding contrasts with Fleeson and Wilt's (2010) results that extraverted behaviour was association with greater authenticity. The influence of the situation is an obvious explanation for the change in trend. A second, not mutually exclusive, explanation is the elimination of positive affect. That is, extraverted behaviour has historically been associated with greater positive affect (Fleeson et al., 2002), and experiencing positive affect has been found to result in feelings of authenticity (Lenton, Slabu, Sedikides, \& Power, 2013). Therefore, the subjective authenticity associated with extraverted behaviour may have been due to extraverted behaviours' association with positive affect 
and not authenticity per se.

The change in pattern compared to previous acting studies suggests that the situation may matter. Unfortunately, there are other potential influences that are novel to this study and they should also be considered. One possibility for the change in patterns in study 2 is the impact of the modified instructions. The description of the desired extraverted behaviour was altered from previous acting studies to be more neutral (i.e., less positive) whereas the introverted behaviour was described more positively than previous acting studies. Based on the affective forecasts, the altered instructions influenced participants' expectations of the impact of their behaviour. More specifically, the modifications may have swayed the condition effect for positive affect and valence by creating the expectations that the acting introverted condition would be more positive. Based on the analyses of forecasted affect, this remains a distinct possibility. Across the studies the positive affect and pleasantness expectations for acting extraverted were similar. Thus, the lack of differences in experienced positive affect may stem from a change in expectations with acting introverted expected to be more positive (closer to the mean) than previous acting studies.

A second possible reason for the change in pattern of results is the role of confederates. The use of confederates in the acting paradigm is not unique to this study. Previous acting studies have employed confederates (study 2 in Zelenski et al., 2012; and study 4 in Zelenski et al., 2013), although in both studies the interaction was between one participant and one confederate. This study is unique by incorporating two confederates engaging with one participant. However, across all three studies, confederates were instructed to respond neutrally. For this study, confederates were specifically instructed 
to control the amount of talking with the goal of keeping the situation relatively quiet. Confederates noted that they found it difficult to respond neutrally while hindering conversation with chatty participants (i.e., when participants were instructed to act extraverted). It is possible that I created a state of cognitive fatigue in my confederates instead of for the participants. Flipping the roles of confederates and participants would contribute greatly to understanding the dynamics of study 2 .

In contrast to this study, previous acting studies have found that confederate and observer ratings have had good agreement with participants' self-reported ratings. Behaviour ratings tend to have the strongest agreement whereas negative affect tends to have the poorest agreement. Importantly, for this study, there was good general agreement between the ratings for extraverted behaviour. Unfortunately, there was a lack of agreement between self-rated and confederate-rated emotions and discrepancies in condition effects between self and confederate-rated emotions. Most notably, there were differing condition effects for positive affect, valence, and self-consciousness. Recall that confederates rated participants in the acting extraverted condition as experiencing more positive affect, more positive valence, and less self-consciousness than participants in the acting introverted conditions. Confederate's lack of agreement on some variables may be due to the introverted nature of the situation which may have resulted in there being less interaction, and thus less opportunity, to observe the participant. Furthermore, the confederates' role of controlling the situation may have been a distraction that compromised observing. The correlations between all confederate-rated variables emphasizes that rating of participants may have been difficult or that nuances may have been missed. 
The multiple modifications made to the acting paradigm resulted in distinctive results compared to other acting studies. Unfortunately, the combination of changes complicates interpretations. Most notably, the lack of association between extraverted behaviour and positive affect allowed for compelling arguments concerning buffering/restoration effects and the influence of positive affect on subjective authenticity. Study 2 results suggest that the situation does matter but perhaps not in the way, or to the extent, originally hypothesized.

\section{General Discussion}

The personality trait of extraversion is one of the best predictors of happiness (Diener \& Seligman, 2002). The relationship is such that, on average, extraverts report more positive affect than introverts (Diener et al., 1999; Lucas \& Fujita, 2000; Pavot et al., 1990). In a distinct approach, Fleeson et al. (2002) demonstrated that behaving extraverted also resulted in greater positive affect, compared to behaving introverted, and this did not depend on disposition (i.e., both trait introverts and trait extraverts reported more positive affect when behaving extraverted). Fleeson et al.'s (2002) results were somewhat surprising in that counter-dispositional behaviour is theorized to have emotional costs (Côté \& Moskowitz, 1998; Little, 1996). Moreover, research on selfregulation suggests that monitoring and modifying behaviour is onerous and depletes a mental resource (Baumeister, 2002; Baumeister et al., 1998).

Subsequent research, employing the acting paradigm developed by Fleeson and colleagues, has explored negative affect, effort, and cognitive fatigue as potential negative consequences for acting extraverted. When found, costs of acting counterdispositionally have been limited to when extraverts have been randomly assigned to act 
introverted (Gallagher et al., 2011; Zelenski et al., 2012). These subsequent studies have consistently reproduced the trend of greater positive affect for acting extraverted relative to acting introverted (Gallagher et al., 2011; McNiel \& Fleeson, 2006; Zelenski et al., 2012, 2013). I proposed one potential explanation for the asymmetrical pattern of previous acting studies, the importance of situational norms. Previous acting studies employed moderately extraverted situations, and thus, contra-situational effects were limited to the acting introverted condition. The goal of this research was to examine the affective and cognitive implications of counter-dispositional behaviours in introverted situations. I tested whether or not the pattern of counter-dispositional consequences found in previous acting research would reverse in introverted situations. That is, I hypothesized that introverts would report more negative affect, greater effort, and demonstrate signs of cognitive fatigue when assigned to act extraverted.

Across two studies, I created introverted situations and participants were randomly assigned to one of three conditions (act extraverted, act introverted, and control conditions). In addition, affect, subjective authenticity, effort, enjoyment measures, and a cognitive task (to assess cognitive fatigue) were administered following a jigsaw puzzle activity. In study 1 , the previous trend of greater positive affect for acting extraverted was re-created. This trend was corroborated by valence and enjoyment. Self-reported effort indicated that acting introverted was more effortful than acting extraverted which was more effortful than the control condition. Furthermore, acting introverted was especially effortful for dispositional extraverts. Despite participants reporting that enacting behaviours was effortful, no evidence for cognitive fatigue was found. Turning to a more novel outcome for acting studies, authenticity results demonstrated that 
extraverted behaviour was not only associated with greater positive affect but that the behaviour was reported to be authentic (although dispositional extraverts reported more authenticity than dispositional introverts for acting extraverted). Introverted behaviour was reported to feel the least authentic and this was especially true for dispositional extraverts.

The hypothesized effects of counter-dispositional costs for combined contrasituational behaviours were not found in study 1 . Of note, the effect sizes for the benefits of acting extraverted (positive affect, valence, and enjoyment) were smaller relative to previous acting studies. This may indicate that the situation does matter, but that the implications of an introverted situation were not as strong as hypothesized or that perhaps the situation was not introverted enough. Furthermore, there were other indications that the situation was less than ideal. Manipulation checks revealed that although participants followed instructions such that conditions differed from each other, the acting extraverted participants did not behave extraverted to the point of being contra-situational, but were more neutral for an introverted situation (i.e., just below the midpoint of the introvertedextraverted scale). A second issue with the situation of study 1 was that participants in the control and acting extraverted conditions interacted and socially isolated participants in the acting introverted condition. The social isolation may have resulted in less positive affect, thus offering a potential explanation for participants in the acting introverted condition reporting lower levels of positive affect relative to participants in the acting extraverted and control conditions. Moreover, participants in the acting extraverted and control conditions may have gained positive friendly feedback from the social interaction 
thus increasing their positive affect and valence. Study 2 addressed these issues by altering the instructions to modify behaviour and employing confederates.

Study 2 results were notably different compared to previous acting studies, including study 1 . In study 2 , the typical trend of greater positive affect for acting extraverted disappeared and was replaced with participants in the acting extraverted condition reporting the least enjoyment and the most effort relative to the other conditions. Participants reported the most subjective authenticity in the control condition and the least authenticity in the acting extraverted condition. Thus, study 2 provides evidence of costs for behaving contra-situationally (acting extraverted in an introverted situation). Despite these contra-situational effects, there were no significant condition by disposition interactions found across all dependent variables for study 2 , and thus results did not support hypotheses regarding costs as a combination of counter-dispositional and contra-situational behaviours. That is, the contra-situational effects, when found, did not differ by disposition.

In addition to a novel (cf. past research) situation, the changes to instructions between study 1 (and previous acting studies) and study 2 also altered participants' expectations about the upcoming activity. Participants expected less positive valence and more negative affect for acting extraverted in study 2 compared to study 1 , and participants expected more positive affect and positive valence for acting introverted in study 2 compared to study 1 . Thus the new instructions successfully highlighted the positive aspects of acting introverted and the unfavourable aspects of acting extraverted. The change in expectations, in combination with the change in affective results, brings the question: would previous research's affective results be different if alternative 
instructions had been used? That is, would the more negative expectation for acting extraverted and more positive expectation for acting introverted create a shift that would eliminate or diminish previous differences between acting conditions? Furthermore, the change in instructions may also have altered how participants enacted. That is, the attributes of extraverted and introverted behaviour may have been distinct for study 2 relative to other acting studies. It could be argued that previous acting results are biased due to differences in positivity between the acting instructions. Alternatively, as positive affect is a central feature of extraversion, it could be argued that highlighting unfavourable aspects of extraversion is not a valid representation of the disposition. Nonetheless, how much the change in expectations about enacting behaviours influenced the experience of enacting the behaviours cannot be determined based on these two studies. That is, it cannot be established whether or not changing expectations actually lead to a different experience in the moment. To explore this idea, a study could have only half of the sample forecast their affect. A difference in experience due to predicting versus not predicting would indicate that reflecting on expectations influenced experience. Alternatively, manipulating participants' expectations prior to an activity (researcher stating how fun versus boring the upcoming activity is) could determine if changing expectations causes a change in experience. Nevertheless, research examining affective forecasting errors (difference between expectations and experience), within the acting paradigm, has established that expectations are not entirely accurate or deterministic (Zelenski et al., 2013).

Study 1 and study 2 are the first studies to assess subjective authenticity following manipulated extraverted behaviour. In study 1, participants in the acting extraverted and 
control conditions reported greater subjective authenticity. This finding agrees with Fleeson and Wilt's (2010) results where extraverted behaviour was associated with greater authenticity. Study 1's authenticity results indicate that participants are able to feel authentic even when their behaviour was manipulated and was somewhat incongruent with the situation. In study 2 , participants in the acting introverted and control conditions reported greater authenticity. Although this could indicate that the situation matters, other aspects of study 2's results suggest that additional factors may be at play in explaining the link between authenticity and extraverted behaviour. More specifically, the links between extraverted behaviour and positive affect may explain its association with authenticity.

A key distinction between study 1 and study 2 is the different findings for positive affect. When extraverted behaviour has been associated with authenticity it is also associated with positive affect. Research shows that positive affect and authenticity are highly correlated (Schlegel et al., 2009), and more recently, experimental positive affect inductions have been shown to cause subjective authenticity (Lenton, Slabu et al., 2013); feeling good feels authentic. When extraverted behaviour does not result in greater positive affect (study 2) there is also a corresponding lack of greater subjective authenticity when compared to acting introverted. This begs the question: was the greater authenticity for extraverted behaviour due to the behaviour per se, or due to the behaviour's association with positive affect? That is, perhaps positive affect produces the relationship between extraverted behaviour and authenticity.

More broadly, results from studies 1 and 2 do not support hypothesized costs for a combination of counter-dispositional and contra-situational behaviour. Study 2 provides 
the greatest evidence for a situational influence with participants in the acting extraverted condition reporting more effort and self-consciousness, and less authenticity and enjoyment, compared to either acting introverted and or the control condition ${ }^{21}$. With results not supporting hypotheses, the three theories that encompass behaviour variability will be revisited with consideration of the results of these two new studies.

Behavioural concordance, free traits, and density distribution of states are three theories that incorporated variability of behaviour. To recap, behaviour concordance postulates that when behaviour and disposition are matched, pleasant affect will result, whereas when behaviour and disposition are mismatched, unpleasant affect will result. The free trait theory also proposes negative costs for counter-dispositional behaviour but differs by including the caveat that counter-dispositional behaviour needs to occur over a prolonged period and that the costs can be in the form of negative impacts to emotional and physical well-being. The density distribution of states differs from both theories in that it does not propose negative consequences for counter-dispositional behaviour. To date, within the acting paradigm, no significant condition by disposition interaction for negative affect has been found. This could suggest that the density distribution of states is correct and that both the behaviour concordance model and free trait theory are incorrect. Interestingly, the behaviour concordance model also posts that there would be pleasant affect for behaviour-disposition congruence. Introverts have not been found to report more pleasant affect when acting introverted. Contrary to this notion, study 1 and previous acting research (Fleeson et al., 2002; McNiel \& Fleeson, 2006; Zelenski et al.,

\footnotetext{
${ }^{21}$ Note that some analyses were only marginally significant.
} 
2012,2013 ) have found that participants report more positive affect and pleasantness in the acting extraverted condition, compared to the acting introverted condition, and this did not depend on disposition (i.e., introverts are also happier acting extraverted).

The lack of significant findings for study 2, for positive affect and pleasantness, are unique but, nevertheless, they do not support the behaviour concordance model. Therefore, findings for both pleasant affect and negative affect conflict with the behaviour concordance model. One important caveat is that the behaviour concordance model is conceived regarding the interpersonal circumplex. The axis of the interpersonal circumplex that is pertinent to the current research is the vertical axis labeled status or power (Wiggins, 1979). This axis is anchored by the terms assertive/dominant versus passive/submissive. In general, the personality dimension of extraversion is broader than the power axis of the interpersonal circumplex. Furthermore, trait extraversion incorporates some features of the agreeableness axis of the interpersonal circumplex (McCrae \& Costa, 1989; Trapnell \& Wiggins, 1990). It is not clear what the implications for this line of research if the more narrow terms of the affect circumplex were utilized instead of the broader extraversion term of the big five. Interestingly, support for behavioural concordance on the power axis has been limited to participants high on dominance reporting less pleasant valance for submissive behaviours. That is, more dominant participants reported either more negative or less positive affect when they behaved submissively. There were no significant associations for the trait of submissiveness (Moskowitz \& Côté, 1995). These results are comparable to the asymmetric pattern of previous acting research. Thus, despite this and other acting 
research not explicitly exploring the interpersonal circumplex, the pattern of results may not have deviated if it had.

There is a similar assessment issue regarding interpreting these results as a direct test of the free trait theory. Specifically, the free trait postulates dispositions as biological. My assessment of trait extraversion was a self-report measure that does not assess the biological basis of personality but adds a subjective element. That is, a biological introvert might self-report more extraversion due to the demands of their job. The free trait theory also proposes costs following behaving out of character for a prolonged period of time. One possibility for the general lack of consequences for counterdispositional behaviour found thus far is that activities have been relatively brief (ranging from ten to twenty minutes). The implications of more long-term behavioural regulation are unknown. Future research might explore cognitive and emotional implications for acting out of character over more prolonged time periods.

Anecdotal evidence does suggest there may be more long-term implications. For example, an undergraduate course in positive psychology was given an optional journal reflection exercise of behaving extraverted for an entire day. Journal entries, for selfidentified introverts, indicated that the task progressively got more difficult throughout the day but that the task was more enjoyable than expected. While suggestive, disposition was not assessed and it is reasonable to assume that there was a self-selection bias to the sample (students that took a positive psychology class and the small subsample that completed the journal activity). Furthermore, despite instructions to complete journal entries immediately after the activity, it is important to acknowledge that there is a strong likelihood that entries were made more retrospectively. Thus, there 
are reasons to doubt these findings as precise. Nonetheless, they do follow hypothesized trends suggested by the free trait theory.

The idea that situations influence behaviour is incorporated in both the free trait theory and the density distribution of states model. The greatest distinction between these two is that the density distribution of states does not posit negative consequences for behaving out of character. According to both theories, behaviour can vary due to influences, other than just disposition, which may include situational properties, the perception of these properties and their impact on situational norms, which in turn can also influence behaviour. Consequently, concepts like self-regulation or contrasituational effects, such as effort, are not necessarily in conflict with these theories (Fleeson, 2007; McCabe \& Fleeson, 2012). Costs found include a significant condition by disposition interaction in study 1 . The pattern was that as trait extraversion increases less effort was reported for acting extraverted (i.e., dispositionally congruent behaviour is easier) and as trait extraversion increases more effort was reported for acting introverted (i.e., dispositionally incongruent behaviour is harder). Thus study 1's interaction indicates that it sometimes feels effortful to behave different from one's disposition. This suggests that the free trait theory notion of consequences for out of character behaviour may have merit. Furthermore, if effort is considered a negative emotional state, then this would suggest the self-concordance model may also have merit. Regrettably, study 2 did not replicate study 1's condition by disposition interaction for effort.

Across both studies, there was limited evidence for condition by disposition interactions. Furthermore, there were minimal negative consequences found for counterdispositional behaviours. Thus, the results are more supportive of the density distribution 
of states model over the free trait theory. Despite the continued lack of findings for negative affect (both for condition and condition by disposition interactions), examining self-consciousness in this research was revealing. For both studies, there was a marginally significant condition effect with participants in the acting extraverted condition reporting more self-consciousness than participants in the acting introverted condition. It is tempting to attribute the greater self-consciousness reported for the acting extraverted condition to the introverted situations employed, but Zelenski et al., (2013) found similar trends in moderately extraverted situations, thus providing evidence that persuades against this conclusion. That is, participants in the acting extraverted condition report more self-consciousness, compared to the other conditions, when the situations were introverted and when the situations were moderately extraverted. Perhaps extraverted behaviour just puts people 'out there' more so that they have more to worry about? The lack of condition by disposition interaction for self-consciousness, across all studies, indicates that the results do not conform to either the behaviour concordance model or free trait theory. The similar pattern of findings, for both introverted and moderately extraverted situations, suggests that how introverted or extraverted the situation is may not play a role in impacting how self-conscious people feel.

This research was motivated in an attempt to explain why introverts don't act extraverted more frequently and to continue explorations of potential negative consequences for acting out of character. The greater self-consciousness reported by introverted participants, compared to extraverted participants, offers one possible explanation for why introverts do not behave extraverted more frequently. Extraverts' characteristic attention seeking has been put forth as the central feature of dispositional 
extraversion (Ashton, Lee, \& Paunonen, 2002). Thus, it follows that extraverted behaviour may also lead to more social attention, compared to introverted behaviour. This greater attention for extraverted behaviour may be a deterrent for individuals that experience more self-consciousness (i.e., introverts). That is, introverts reporting more self-consciousness in combination with extraverted behaviour gaining more social attention may reach a threshold that is undesirable. Thus, self-consciousness could be a form of negative consequence for extraverted behaviour.

Although introverts reported more self-conscious affect across conditions, Zelenski et al. (2013) has also found that introverts, incorrectly, forecast even more selfconsciousness than they experienced. That is, based on my results, dispositional introverts do experience more self-consciousness than their extraverted counterparts but they may also expect to experience an even greater amount than they actually experience. Experiencing and expecting to experience greater self-consciousness both offer avenues for future exploration and potential explanations for why introverts do not behave extraverted more frequently.

Coming back to the questions of why extraverts are happier and why extraverted behaviour is associated with positive affect, socializing offers one possible explanation. Socializing has been put forth as a mechanism explaining why extraverts are happier. Social interactions have been found to increase positive affect (Clark \& Watson, 1988; McIntyre, Watson, Clark, \& Cross, 1991; Vittengl \& Holt, 2000), and extraverts engage in more social interactions compared to introverts (Argyle \& Lu, 1990; Watson et al., 1992). Three distinct ESM studies have found that social interactions partially mediate the relationship between trait extraversion and aggregated positive affect (Lucas, Le, \& 
Dyrenforth, 2008; Srivastava, Angelo, \& Vallereux, 2008) indicating that the frequency of extraverts' social interactions cannot completely explain their higher levels of positive affect. In a conceptually similar study, state behaviour and state positive affect have been explored as a partial explanation for the trait extraversion and trait positive affect relationship (Wilt et al., 2012). Wilt et al. (2012) differ from Lucas et al. (2008) and Srivastava et al. (2008) by examining state behaviour (i.e., rating on extraversion adjectives) versus social interactions (i.e., alone, interacting with others, or interacting with multiple others) as mediators to the trait extraversion-trait positive affect association. Thus, Wilt and colleagues (2012) explored if more frequent enactment of extraverted behaviour resulted in more frequent state positive affect, which in turn lead to greater trait positive affect. Hence, Wilt et al. (2012) tested if one reason for dispositional extraverts' greater positive affect is that they enact extraverted behaviour more frequently (i.e., it is not what extraverts passively have that makes them happier, but what they $d o$ ). In support for their stance, Wilt and colleagues found, across 5 ESM studies, that extraverted behaviour partially ${ }^{22}$ mediated the relationship between trait extraversion and trait positive affect. Across studies socializing and extraverted behaviour appear to be a partial mediators of the trait extraversion-positive affect link (Lucas et al., 2008; Srivastava et al., 2008; Wilt et al., 2012). Lucas and colleagues note that people are happier when socializing, but that trait extraversion did not moderate this trend indicating that both introverts and extraverts get similar benefits from engaging in social activities.

${ }^{22}$ Wilt et al (2012) studies 1, 3, and 6 found the path between trait extraversion and trait positive affect to be fully mediated when state extraversion and state positive affect were entered into the model. Studies 2 and 4 found partial mediation. The term partial was selected as the more conservative result across the studies. 
One distinction between the findings is that the amount of variability accounted for in the extraversion-positive affect link was greater for extraverted behaviour compared to social interactions. This could suggest that there may be something unique about the behaviour within social interactions that simply being in a social context does not necessarily provide.

Nonetheless, if participating in a social interaction is similarly rewarding, compared to acting extraverted, cajoling introverts into being social may be easier than persuading them to act extraverted. No known study that has tested the difference in adherence to instructions (be social versus behave extraverted) and this offers an avenue for future exploratory research. However, there are suggestions that extraverted behaviour within social interactions may offer greater rewards than simply attending social interactions. Additional evidence for the behavioural component as a critical element for eliciting positive affect comes from a study where participants were instructed to be efficient or social (smile, make eye contact, or engage in a brief conversation) when ordering a Starbucks beverage. Participants in the social condition reported more positive affect than participants in the efficient condition (Sandstrom \& Dunn, 2013). Importantly, this study conceptually replicates previous acting extraverted studies' link to greater positive affect, and extends the results to a real-world setting without explicit instructions to act extraverted.

Another avenue by which socializing may cause positive affect is through friendly feedback. That is, perhaps extraverted behaviour earns more friendly responses and the positive social feedback is inherently rewarding. In a preliminary study, González (2013) explored friendly feedback for extraverted behaviour by having confederates act 
extraverted or act introverted when completing an activity with a participant. Participants and video coders both indicated that participants' friendly behaviour did not differ across acting conditions, casting doubt on the feedback explanation. Conversely, confederates perceived more favourable reactions from participants when they were acting extraverted versus when they acted introverted. Thus, it may be that extraverted behaviour does not actually earn more positive feedback, but rather a perception of friendly responses that leads to increases in positive affect. One important caveat is that the confederates in González's (2013) study may not have been naïve to the premise of the research. Thus, it is possible that confederates ratings of participant's friendly behaviour stems from their knowledge of the research design and would not generalize to social situations outside the lab.

Returning to my results, one justification for study 1's link between extraverted behaviour and positive affect, expressed earlier in this document, was positive feedback (or social isolation of participants in the acting introverted condition), and this motivated the use of confederates for study 2. It is not clear if participants in study 2 's acting extraverted condition may still have perceived confederates' responses as more friendly. That is, confederates' behaviour may have been highly similar across conditions, but the perception of their friendly responses may still have differed by condition. Unfortunately, I did not ask participants to rate how friendly they perceived fellow activity members' behaviour in either study. Despite being contrary to González results, it remains possible that eliminating the positive affect-extraverted behaviour association in study 2 may be entirely, or partially, due to confederates' consistent neutral responses and lack of friendly responses. In support of this idea, there was a smaller effect size for positive 
affect in study 1 compared to previous acting research. Introverted situations most likely provide less opportunity for social interaction (and thus also less opportunity for positive feedback). Thus, the smaller effect size may stem from the reduced positive feedback from the introverted situation, and may explain why social situations are generally more pleasant than non-social situations (Lucas \& Diener, 2001; Whelan \& Zelenski, 2012).

One final explanation for the link between extraverted behaviour and greater positive affect is (introverted) participants' relief that they are finished acting. That is, a possible explanation for why introverts report greater positive affect following acting extraverted is that they are relieved that the experience/activity is over. It is intriguing that study 2 is an anomaly in the positive affect-extraverted behaviour association and it is also where participants were specifically deceived that they would continue the puzzle activity (and thus the behavioural aspects associated with the activity) following completion of the questionnaires and the Stroop task. The lack of positive affect findings in combination with instructions indicating that the task would continue suggests there may be merit in a change to affect due to conclusion of enacting the behaviours. On the other hand, previous research has explicitly asked participants how they felt during the activity. Thus, the emotional implications of finishing the enacting component of the study should have been minimized or non-existent. Future research may wish to explore this avenue.

Extraverted behaviour has also been explored for links with authenticity (Fleeson \& Wilt, 2010). In both real-world and lab contexts, they found a positive association between extraverted behaviour and self-reported authenticity. Furthermore, the association did not depend on disposition, indicating that both introverts and extraverts 
reported greater authenticity when behaving extraverted. My studies explored if manipulated extraverted behaviour in an introverted situation would still be associated with greater subjective authenticity, relative to participants behaving introverted. Study 1 found participants in the acting extraverted condition reporting greater authenticity, relative to participants in the acting introverted condition, even though the situation was relatively introverted. Furthermore, the high degree of similarity between control condition and acting extraverted condition suggests that participants can feel authentic about their manipulated behaviour even when that behaviour is contra-situational. Conversely, study 2 had participant in the acting extraverted condition report the least authenticity, and this did not differ by disposition, suggesting a contra-situational effect.

The two studies presented here represent the first studies that have examined subjective authenticity following manipulated extraverted behaviour. The variation in findings between the two studies prevents any firm conclusions about the role of situations and/or acting instructions on authenticity. Turner and Billings (1991) explored state authenticity and found that situational contexts conducive to authenticity are characterized by acceptance and openness, whereas inauthentic situations were characterized by superficial sociability and awkwardness. For study 1, the social isolation of participants in the acting introverted condition may have felt awkward, whereas, for study 2 , participants in the acting extraverted condition, unsuccessfully trying to socialize with the confederates, may have been left feeling that their responses were superficial.

Lenton, Bruder et al (2013) note that characteristics of authentic situations are more likely to involve achievements, socializing, and fun, whereas inauthentic situations 
involved experiencing pressure from others, being evaluated, and failing to meet objectives. Their findings, despite not examining positive affect or effort explicitly, offer support for these conceptual links to authenticity. Thus, one possibility for why extraverted behaviour has previously been associated with authenticity is that the situations where extraverted behaviour occurred may have been more social and fun. Furthermore, acting extraverted would be more social and fun compared to acting introverted. Comparing studies, study 2's use of individual puzzles, rather than the group puzzle used in study 1, may have created a more evaluative context, in that individual progress could be readily determined, ${ }^{23}$ and thus created a situation that was more inauthentic. Moreover, study 2 included explicit goals that would have provided additional assessment criteria potentially amplifying the evaluative aspects and thus making the situation more inauthentic. The divergence in authenticity between study 1 and study 2 also reveals possible processes through which extraverted behaviour may be linked to authenticity; namely positive affect and effort.

The coinciding change in positive affect between study 1 and study 2 tangles the interpretation, yet suggests that positive affect may underlie participants' subjective authenticity. That is, feeling good feels authentic. Positive affect and authenticity are highly correlated (Schlegel et al., 2009) and inducing positive affect has been shown to results in feelings of authenticity (Lenton, Slabu et al., 2013). Across both my studies, there were strong correlations between authenticity and positive affect. Thus, positive

${ }^{23}$ In study 2, participants in the acting extraverted condition were not necessarily concerned with their own progress but preventing the progress of the other session members which the independent puzzle task, relative to the group puzzle task, also provided. 
affect may have resulted in authenticity. Study 1 and 2 had strong negative correlations between authenticity and effort indicating easier behaviour was reported as being more authentic. Unfortunately, the effort and authenticity items were listed on the same sheet of paper and this may have artificially created or inflated their association. Alternatively, for study 2, perhaps trying to prevent the confederates from completing their puzzles was inauthentic for most participants and required additional effort on top of that for acting. As a first step of disentangling authenticity and effort, I suggest separating the scale items. Future research on authentic behaviours could examine if the concept of effort can be disentangled from authentic behaviour. That is, can participants feel inauthentic for easy behaviour or authentic for effortful behaviours?

An avenue for expanding authenticity research is to draw on trait authenticity. More specifically the behavioural subscale of the authenticity inventory (AI3; Goldman \& Kernis, 2004) is designed to assess the tendency to behave in accordance with values and ideas. It is not known, in my studies, if participants valued behavioural consistency. It is possible that participants high on behavioural consistency did not feel authentic about their counter-dispositional behaviour even when it was extraverted. Alternatively, if authenticity corresponds to values, Whelan and Zelenski (2012b) show that $62 \%$ of a student sample perceived their ideal-self as more extraverted. Thus, extraverted behaviour may be valued by the majority of my sample and could explain the trends found in study 1. This ideal-self view may bias interpretations of extraverted behaviour such that the behaviour is internalized and behaving in a more ideal manner may be perceived as favourable and authentic. Sheldon et al. (1997) argue, via selfdetermination theory, that the personality traits of extraversion, openness, agreeableness, 
conscientiousness, and emotional stability may be linked to authenticity because they support autonomy. Following the density distribution of states rational, personality traits are the state behaviour that we display most frequently. Thus, Sheldon et al.'s (1997) argument should also apply to momentary behaviour too. Perhaps this is one reason for why acting extraverted has been associated with authenticity. This would be especially true when behaviour was self-selected as behavioural instructions potentially undermine autonomy. Moreover, perhaps the mole instructions (preventing other participants from completing their puzzle) in the acting extraverted condition for study 2 might have also had people violate other values (fairness or being nice to people) that further undermined autonomy and thus also eliminated feelings of authenticity.

\section{Strengths and Limitations}

A key strength of this research is that both studies successfully created introverted situations in the lab with the situations still enabling participants to enact both extraverted and introverted behaviour. Using the acting paradigm of previous research was essential to compare findings from moderately extraverted situations previously employed with the introverted situations of this research. Use of a lab context is both advantageous and problematic. The use of a lab environment, compared to a real world setting, allows researchers to control extraneous variables. More importantly, it provided a more direct comparison to previous research as multiple acting studies have occurred in the specific lab that was used for this research (both studies in Zelenski et al., 2012; and studies 2 through 5 from Zelenski et al., 2013). Having studies occur in the same location would have minimized the impact of alternative environments and permit a more equivalent comparison across these studies. It is also possible that the context of a lab may have 
restricted the range of behaviours that participants would enact, further complicating research in this line of inquiry. An idea that I strongly debated was to conduct the present research in an alternative location. More specifically, to have study 1 and 2 take place in a study room within the university library instead of in the lab. The library setting was perceived to allow introverted behaviours to thrive and discourage extraverted behaviours thus providing an ideal situational norm for this research. Unfortunately, the university library was scheduled to undergo an expansion and was a construction zone during the data collection period, thus providing a less than ideal context for data collection in an introverted situation.

Unfortunately, the lab context may create additional expectations about behaviour and may also limit the range of behaviours participants' engage in. One particular journal entry from the positive psychology class, mentioned earlier, highlights these issues. In attempting the acting extraverted for a day activity, he noted that his extraverted behaviour with a friend in the library was considerably different from his extraverted behaviour at a social gathering later that evening. He explained this discrepancy by the influence of situational norms affecting the range of behaviours he was willing (or able) to attempt. Across both situations he felt like he was behaving extraverted, relative to what he would normally have done. Thus, not only may the situation affect what participants are willing to do but it may also influence how the behaviour would be perceived. That is, the exact same behaviour occurring in two different situations may receive two different ratings. One possible way of determining the extent to which observer ratings change due to situations is to employ actors to engage with participants across a variety of situations. Actors could be trained so that 
their behaviour is highly consistent despite a change to situational context. Ratings for the same behaviours in different contexts could then be examined to determine the extent to which observers may be influenced by context.

Another strength of this research was the collection of multiple dependent variables, such as effort, enjoyment, self-consciousness, Stroop, and authenticity. The variety of the dependent variables (affective, cognitive, self-report, observer-rated, etc.) provided a more nuanced perspective of implications for enacted behaviours. Despite creating two introverted situations, the pattern of findings differed between the two studies. Thus, it is difficult to ascertain which results would replicate in future research. Furthermore, as the majority of acting research has employed moderately extraverted situations, the limited range of situations makes predictions to effects in different contexts problematic. This research extended the range of situations to introverted contexts. Extending the range in the opposite direction (more extreme extraverted situation) would provide critical information regarding the generalizability of the benefits for acting extraverted that have been found in moderately extraverted situations. It is possible that the trends from moderately extraverted situations would not replicate in more extraverted environments, thus adding additional caveats to the benefits of introverts acting extraverted.

A key limitation of this research is that the effects found may not be generalizable. All participants were undergraduate students who, as young adults, may be more comfortable trying out different behavioural styles to determine who they are and this may be especially true when interacting with strangers. It is not known if the same patterns would be found with older adults. Additionally, changes to the enacting 
timeframes may have implications to the results. That is, both studies had participants act for brief periods of time and consequences may differ if the acting periods were longer or shorter. Furthermore, the trends found may not replicate or occur in real world contexts. And finally, all acting studies have occurred in a North American context where trait extraversion is associated with characteristics that are desired.

Despite participants reporting more effort for enacting behaviours, there were no significant findings for Stroop analyses in spite of the fact that multiple techniques were attempted. Thus, self-reported effort trends were not substantiated by an objective measure. Possible explanations include that all participants were equally depleted by the activity, no participants were depleted by the activity, or perhaps the effects of counterdispositional and contra-situational behaviours are more affective and not cognitive. Notwithstanding the appeal of this last point, it is noteworthy that Gallagher et al. (2010) assessed ego depletion with a non-cognitive measure (hand-grip) and also had null findings. Thus, it seems more likely that some other variable is masking or eliminating effects that would corroborate effortful feelings. Alternatively, there may not be ego depletion for contra-situational and counter-dispositional effects for acting extraverted. Regardless, future research may wish to incorporate additional objective measures of self-regulation and stress. Cortisol and interlukin-6 (IL-6) are released when people are under stress and can be measured in saliva and blood (Maes et al., 1998; Michaud, Matheson, Kelly, \& Anisman, 2008; Levine, Zagoory-Sharon, Feldman, Lewis, \& Weller, 2007; Sjögren, Leanderson, Kristenson, \& Emerudh, 2005). An acting study with multiple saliva or blood samples could reveal a trend different from subjective findings and thus provide critical insight into why introverts don't act extraverted more often. 


\section{Conclusions}

This research was motivated to extend the acting extraverted research paradigm to introverted situations and thus, determine if previous affective and cognitive trends of acting extraverted would be found in introverted situations. Despite the intuitive appeal that acting out of character will result in negative consequences, research has largely not supported a costs perspective. There have now been multiple studies assessing a condition by disposition interaction on self-reported affect. Zelenski et al. (2013) combined four separate studies to provide greater power and did not find significant interactions of condition by disposition on positive, negative, or pleasant affect. It is noteworthy that there are no known studies that have attempted the acting paradigm in an extreme extraverted situation and introverted situations are limited to the two studies presented here. The lack of condition by disposition interaction may stem from the

combination of limited situation diversity and possible restricted range of behaviours within the lab context. Based on the current enacting paradigm, finding a significant condition by disposition effect in the lab seems improbable. ESM studies have also failed to find behaviour by disposition interactions thus suggesting that there may not be an interaction effect to be found.

Across eleven known acting studies, where extraverted behaviour was manipulated, participants in the acting extraverted condition reported more positive affect compared to participants in the acting introverted condition in ten of these studies. My study 2 is the single exception to this trend indicating there may be a general robustness and multiple potential mechanisms underlying the effect. Experience sampling methodology, where behaviour and affect are measured at a state level, has also found 
this trend (Fleeson et al., 2002; McNiel \& Fleeson, 2006). Potential mechanisms underlying the positive affect extraverted behaviour association include, but are not limited to, socializing, friendly feedback, autonomy, and relief following activity completion. Furthermore, a possible reason for why introverts do not behave extraverted was ascertained, namely greater experiences and expectation for feeling self-conscious. Given the large amount of data that demonstrates benefits for acting extraverted, introverts truly may be better off (i.e., happier) if they behaved extraverted more often. Study 2 provides a critical caveat to this in that it may not always be beneficial to act extraverted. 


\section{References}

Aguinis, G. (2004). Regression analysis for categorical moderators. New York, NY: Guilford Press.

Anderson, N. H. (1968). Likableness ratings of 555 personality-trait words. Journal of Personality and Social Psychology, 9, 272-279. doi: 10.1037/h0025907

Argyle, M., \& Lu, L. (1990). Happiness and social skills. Personality and Individual Differences, 11, 1255-1261. doi: 10.1016/0191-8869(90)90152-H

Ashton, M. C., Lee, K., \& Paunonen, S. V. (2002). What is the central feature of extraversion? Social attention versus reward sensitivity. Journal of Personality and Social Psychology, 83, 245-252. doi: 10.1037/0022-3514.83.1.245

Bauer, I. M., \& Baumeister, R. M. (2011). Self-regulatory strength. In K. D. Vohs and R. F. Baumeister (Eds.), Handbook of self-regulation: Research, theory, and applications (2nd ed., pp. 64-82). New York, NY: The Guilford Press.

Baumeister, R. F. (2002). Ego depletion and self-control failure: An energy model of the self's executive function. Self and Identity, 1, 129-136. doi: $10.1080 / 152988602317319302$

Baumeister, R. F., Bratslavsky, E., Muraven, M., \& Tice, D. M. (1998). Ego depletion: Is the active self a limited resource? Journal of Personality and Social Psychology, 74, 1252-1265. doi: 10.1037/0022-3514.74.5.1252

Baumeister, R. F., Gailliot, M., Dewall, N. C., \& Oaten, M. (2006). Self-regulation and personality: How interventions increase regulatory success, and how depletion moderates the effects of traits on behavior. Journal of Personality, 74, 1773-1801. doi: 10.1111/j.1467-6494.2006.00428.x 
EXTRAVERSION AND COUNTER-DISPOSITIONAL BEHAVIOUR

Baumeister, R. F., Vohs, K. D., \& Tice, D. M. (2007). The strength model of self-control. Current Directions in Psychological Science, 16, 396-403. doi: 10.1111/j.14678721.2007.00534.x

Carver, C. S., Sutton, S. K., \& Scheier, M. F. (2000). Action, emotion, and personality: Emerging conceptual integration. Personality and Social Psychology Bulletin, 26, 741-751. doi: $10.1177 / 0146167200268008$

Carver, C. S., \& White, T. L. (1994). Behavioral inhibition, behavioral activation and affective responses to impending reward and punishment: The BIS/BAS scales. Journal of Personality and Social Psychology, 67, 319-333. doi: 10.1037/00223514.67.2.319

Charles, S. T., Reynolds, C. A., \& Gatz, M. (2001). Age-related differences and change in positive and negative affect over 23 years. Journal of Personality and Social Psychology, 80, 136-151. doi: 10.1037/0022-3514.80.1.136

Clark, L. A., \& Watson, D. (1988). Mood and the mundane: Relations between daily life events and self-reported mood. Journal of Personality and Social Psychology, 54, 296-308. doi: 10.1037/0022-3514.54.2.296

Cooper, W. H., \& Withey, M. J. (2009). The strong situation hypothesis. Personality and Social Psychological Review, 13, 62-72. doi: 10.1177/1088868308329378

Costa, P.T., Jr., \& McCrae, R. R. (1980). Influence of extraversion and neuroticism on subjective well-being. Journal of Personality and Social Psychology, 38, 668678. doi: $10.1037 / 0022-3514.38 .4 .668$

Costa, P. T., Jr., \& McCrae, R. R. (1989). The NEO-PI/NEO-FFI manual supplement. Odessa, FL: Psychological Assessment Resources. 
Côté, S., \& Moskowitz, D. S. (1998). On the dynamic covariation between interpersonal behavior and affect: Prediction from neuroticism, extraversion, and agreeableness. Journal of Personality and Social Psychology, 75, 1032-1046. doi: 10.1037/00223514.75.4.1032

Cunningham, M. R. (1988). Does happiness mean friendliness? Induced mood and heterosexual self-disclosure. Personality and Social Psychology Bulletin, 14, 283 297. doi: $10.1177 / 0146167288142007$

Deci, E. L., \& Ryan, R. M. (1995). Human autonomy: The basis for true self-esteem. In M. H. Kernis (Ed.) Efficacy, agency, and self-esteem (p. 31-49). Plenum Press.

Deci, E. L., \& Ryan, R. M. (2000). The "what" and "why" of goal pursuits: Human needs and the self-determination of behavior. Psychological Inquiry, 11, 227-268. doi: 10.1207/S15327965PLI1104_01

De Young, C. G. (2006). Higher-order factors of the Big Five in multi-informant sample. Journal of Personality and Social Psychology, 91, 1138-1151. doi: 10.137/00223514.91.6.1138

Diener, E., Larsen, R. J., Levine, S., \& Emmons, R. A. (1985). Intensity and frequency: Dimensions underlying positive and negative affect. Journal of Personality and Social Psychology, 48, 1253-1265. doi: 10.1037/0022-3514.48.5.1253

Diener, E., \& Seligman, M. E. P. (2002). Very happy people. Psychological Science, 13, 81-84. doi: 10.1111/1467-9280.00415

Diener, E., Suh, E. M., Lucas, R. E., \& Smith, H. L. (1999). Subjective well-being: Three decades of progress. Psychological Bulletin, 125, 276-302. doi: 10.1037/00332909.125.2.276 
Digman, J. M. (1990). Personality structure: Emergence of the Five-Factor Model. Annual Review of Psychology, 41, 417-440. doi: 10.1146/annurev.psych.41.1.417

Digman, J. M. (1997). Higher-order factors of the Big Five. Journal of Personality and Social Psychology, 73, 1246-1256. doi: 10.1037/0022-3514.73.6.1246

Emmons, R. A., Diener, E., \& Larsen, R. J. (1985). Choice and avoidance of everyday situations and affect congruence: Two models of reciprocal interactionism. Journal of Personality and Social Psychology, 51, 815-826.

Fischer, H., Wik, G., \& Fredrikson, M. (1997). Extraversion, neuroticism and brain function: A pet study of personality. Personality and Individual Differences, 23, 345-352. doi: 10.1016/S0191-8869(97)00027-5

Fleeson, W. (2001). Towards a structure- and process-integrated view of personality: Traits as density distributions of states. Journal of Personality and Social Psychology, 80, 1011-1027. doi: 10.1037/0022-3514.80.6.1011

Fleeson, W. (2004). Moving personality beyond the person-situation debate: The challenge and the opportunity of within-person variability. Current Directions in Psychological Science, 13, 83-87. doi: 10.1111/j.0963-7214.2004.00280.x

Fleeson, W. (2007). Situation-based contingencies underlying trait-content manifestation in behavior. Journal of Personality, 75, 825-861. doi: 10.1111/j.14676494.2007.00458.x

Fleeson, W., \& Gallagher, M. P. (2009). The implications of Big-Five standing for the distribution of trait manifestation in Behavior: Fifteen experience-sampling studies and a meta-analysis. Journal of Personality and Social Psychology, 97, 1097-1114. doi:10.1037/a0016786 
Fleeson, W., \& Leicht, C. (2006). On delineating and integrating the study of variability and stability in personality psychology: Interpersonal trust as illustration. Journal of Research in Personality, 40, 5-20. doi: 10.1016/j.jrp.2005.08.004

Fleeson, W., Malanos, A. B., \& Achille, N. M. (2002). An intraindividual process approach to the relationship between extraversion and positive affect: Is acting extraverted as "good" as being extraverted? Journal of Personality and Social Psychology, 83, 1409-1422. doi: 10.1037/0022-3514.83.6.1409

Fleeson, W., \& Noftle, E. E. (2009). In favor of the synthetic resolution to the personsituation debate. Journal of Research in Personality, 43,150-154. doi: 10.1016/j.jrp.2009.02.008

Fleeson, W., \& Wilt, J. (2010). The relevance of Big Five trait content in behavior to subjective authenticity: Do high levels of within-person behavioral variability undermine or enable authenticity achievement? Journal of Personality, 78, 13531382. doi: 10.1111/j.1467-6494.2010.00653.x

Funder, D. C. (2006). Towards a resolution of the personality triad: Persons, situations, and behaviors. Journal of Research in Personality, 40, 21-34. doi: 10.1016/j.jrp.2005.08.003

Funder, D. C. (2009). Persons, behavior and situations: An agenda for personality psychology in the postwar era. Journal of Research in Personality, 43, 120-126. doi: 10.1016/j.jrp.2008.12.041

Funder, D. C., (2013, January). Taking the power of the situation seriously. Presidential Address at the meeting of the Society for Personality and Social Psychology, New Orleans, LA. 
Furr, R. M., \& Funder, D. C. (2004). Situational similarity and behavioral consistency: Subjective, objective, variable-centered, and person-centered approaches. Journal of Research in Personality, 38, 421-447. doi: 10.1016/j/jrp.2003.10.001

Galton, F. (1884). Measurement of character. Fortnightly Review, 36, 179-185.

Gallagher, P., Fleeson, W., \& Hoyle, R. H. (2011). A self-regulatory mechanism for personality trait stability: Contra-trait effort. Social Psychological and Personality Science, 2, 335-342. doi: 10.1177/1948550610390701

Gillen, N. R. (2009). The effects of acting counter-dispositionally on cognition and mood (Undergraduate thesis). Unpublished, Department of Psychology, Carleton University, Ottawa, Canada.

Goldberg, L. R., (1990). An alternative "description of personality": The Big-Five factor structure. Journal of Personality and Social Psychology, 59, 1216-1229. doi: $10.1037 / 0022-3514.59 .6 .1216$

Goldberg, L. R. (1992). The development of markers for the Big-Five factor structure. Psychological Assessment, 4, 26-42. doi: 10.1037/1040-3590.4.1.26

Goldberg, L. R. (1993). The structure of phenotypic personality traits. American Psychologist, 48, 26-34. doi:10.1037//0003-066X.48.1.26

Goldman, B. M., \& Kernis, M. H. (2004). The Authenticity Inventory-Version 3 (AI-3). In M. P. Zanna (Ed.), Advances in Experimental Social Psychology, (Vol. 38, 2006, pp. 345-348). San Diego, CA: Academic Press.

González, A. (2013). An investigation of social responses to extraverted behaviour. (Undergraduate thesis). Unpublished, Department of Psychology, Carleton University, Ottawa, Canada. 
Gray, J. A. (1970). The psychophysiological basis of introversion- extraversion.

Behavior Research and Therapy, 8, 249-266. doi: 10.1016/0005-7967(70)90069-

0

Gray, J. A. (1972). The psychophysiological basis of introversion-extraversion: A modification of Eysenck's theory. In V. D. Nebylitsyn \& J. A. Gray (Eds.), The biological basis of individual behaviour (pp. 182-205). San Diego, CA: Academic Press.

Gray, J. A. (1981). A critique of Eysenck's theory of personality. In H. J. Eysenck (Ed.), A model of personality (pp.246-276). Berlin, Germany: Springer-Verlag.

Hagger, M. S., Wood, C., Stiff, C., \& Chatzisarantis, N. L. (2010). Ego depletion and the strength model of self-control: A meta-analysis. Psychological Bulletin, 136, $495-$ 525. doi: 10.1037/a0019486

Harter, S. (2002). Authenticity. In C. R. Snyder, and S. J. López (Eds.), Handbook of positive psychology (pp. 382-394). New York, NY: Oxford University Press.

Hills, P., \& Argyle, M. (2001). Happiness, introversion-extraversion and happy introverts. Personality and Individual Differences, 30, 595-608. doi: $10.1016 / \mathrm{S} 0191-8869(00) 00058-1$

Hogan, R., Harkness, A. R., \& Lubinski, D. (2000). Personality and individual differences. In K. Pawlik and M. R. Rosenzweig (Eds.), International handbook of psychology (pp. 283-304). London, England: Sage.

Kernis, M. H., \& Goldman, B. M. (2005). Authenticity, social motivation, and psychological adjustment. In J. P. Forgas, K. D. Williams, \& S. M. Laham (Eds.), Social motivation: Conscious and unconscious processes (pp. 210-227). New 
York, NY: Cambridge University Press.

Kirby, D. M., \& Gardner, R. C. (1972). Ethnic stereotypes: Norms of 208 words typically used in their assessment. Canadian Journal of Psychology, 26, 140-154. doi: $10.1037 / \mathrm{h} 0082423$

Krueger, J. I. (2009). A componential model of situation effects, person effects, and situation-by-person interaction effects on social behavior. Journal of Research in Personality, 43, 127-136. doi: 10.1016/j.jrp.2008.12.042

Larsen, R. J., \& Diener, E. (1992). Promises and problems with the circumplex model of emotion. In M. S. Clark (Ed.), Review of Personality and Social Psychology, 13, 25-59. Thousand Oaks, CA: Sage Publications.

Larsen, R. J., \& Ketelaar, T. (1991). Personality and susceptibility to positive and negative emotional states. Journal of Personality and Social Psychology, 61, 132140. doi: $10.1037 / 0022-3514.61 .1 .132$

Larsen, J. T., McGraw, A. P., \& Cacioppo, J. T. (2001). Can people feel happy and sad at the same time? Journal of Personality and Social Psychology, 81, 684-696. doi: $10.1037 / 0022-3514.81 .4 .684$

Lenton, A. P., Bruder, M., Slabu, L., \& Sedikides, C. (2013). How does “being real” feel? The experience of state authenticity. Journal of Personality, 81, 276-289. doi: 10.1111/j.1467-6494.2012.00805.x

Lenton, A., Slabu, L., Sedikides, C., Power, K., (2013). I feel good, therefore I am real: Testing the causal influence of mood on state authenticity. Cognition and Emotion, 27, 1202-1224. doi: 10.1080/02699931.2013.778818

Levine, A., Zagoory-Sharon, O., Feldman, R., Lewis, J. G., \& Weller, A. (2007). 
EXTRAVERSION AND COUNTER-DISPOSITIONAL BEHAVIOUR

Measuring cortisol in human psychobiological studies. Physiology and Behavior, 90, 43-53. doi: 10.1016/j.physbeh.2006.08.025

Lischetzke, T., \& Eid, M. (2006). Why extraverts are happier than introverts: The role of mood regulation. Journal of Personality, 74, 1127-1161. doi: 10.1111/j.14676494.2006.00405.x

Little, B. R. (1983). Personal Projects: A rational and method for investigation. Environment and Behavior, 15, 273-309. doi: 10.1177/001391658153002

Little, B. R. (1996). Free traits, personal projects and idio-tapes: Three tiers for personality psychology. Psychological Inguiry, 7, 340-344. doi: 10.1207/s15327965pli0704_6

Little, B. R. (2000). Free traits and personal contexts: Expanding a social ecological model of well-being. In W. B. Walsh, K. H. Craik \& R. H. Price (Eds.), Personenvironment psychology: New directions and perspectives (2nd ed., pp. 87-116). Mahwah, NJ: Lawrence Erlbaum Associates.

Little, B. R. (2008). Personal projects and free traits: Personality and motivation reconsidered. Social and Personality Psychology Compass, 2, 1235-1254. doi: 10.1111/j.1751-9004.2008.00106.x

Little, B. R., \& Joseph, M. F. (2007). Personal projects and free traits: Mutable selves and well-beings. In B. R. Little, K. Salmela-Aro, \& S. D. Phillips (Eds.), Personal project pursuit: Goals, actions, and human flourishing (pp. 373-398). Mahwah, NJ: Lawrence Erlbaum.

Lucas, R. E., \& Baird, B. M. (2004). Extraversion and emotional reactivity. Journal of Personality and Social Psychology, 86, 473-485. doi: 10.1037/0022- 
3514.86.3.473

Lucas, R. E., \& Diener, E. (2001). Understanding extraverts' enjoyment of social situations: The importance of pleasantness. Journal of Personality and Social Psychology, 81, 343-356. doi: 10.1037/0022-3514.81.2.343

Lucas, R. E., \& Donnellan, M. B. (2009). If the person-situation debate is really over, why does it still generate so much negative affect? Journal of Research in Personality, 43, 146-149. doi: 10.1016/j.jrp.2009.02.009

Lucas, R. E., \& Fujita, F. (2000). Factors influencing the relation between extraversion and pleasant affect. Journal of Personality and Social Psychology, 79, 1039-1056. doi: $10.1037 / 0022-3514.79 .6 .1039$

Lucas, R. E., Le, K., \& Dyrenforth, P. S. (2008). Explaining the extraversion/positive affect relation: Sociability cannot account for extraverts greater happiness. Journal of Personality, 76, 385-414. doi: 10.1111/j.1467-6494.2008.00490.x

Maes, M., Song, C., Lin, A., De Jongh, R., Van Gastel, A., Kenis, G., ... \& Smith, R. S. (1998). The effects of psychological stress on humans: Increased production of pro-inflammatory cytokines and Th1-like response in stress-induced anxiety. Cytokine, 10, 313-318. doi: 10.1006/cyto.1997.0290

Maslow, A. H. (1968). Toward a psychology of being (2nd ed.). Princeton, HN: Van Nostrand.

Matthews, G., Deary, I. J., \& Whiteman, M. C. (2003). Personality traits (2nd ed.) New York, NY: Cambridge University Press.

McCabe, K. O., \& Fleeson, W. (2012). What is extraversion for? Integrating trait and motivational perspectives and identifying the purpose of extraversion. 
Psychological Science, 23, 1498-1505. doi: 10.1177/0956797612444904

McCrae, R. R., \& Costa, P. T., Jr. (1991). Adding liebe und arveit: The full Five-Factor model and well-being. Personality and Social Psychology Bulletin, 17, 227-232. doi: $10.1177 / 014616729101700217$

McCrae, R. R., \& Costa, P. T., Jr. (1989). The structure of interpersonal traits: Wiggin's Circumplex and the Five-Factor model. Journal of Personality and Social Psychology, 56, 586-595. doi: 10.1037/0022-3514.56.4.586

McIntyre, C. W., Watson, D., Clark, L. A., \& Cross, S. A. (1991). The effect of induces social interaction on positive and negative affect. Bulletin of the Psychonomic Society, 29, 67-70. doi: 10.3758/BF03334773

McNiel, J. M., \& Fleeson, W. (2006). The causal effects of extraversion on positive affect and neuroticism on negative affect: Manipulating state extraversion and state neuroticism in an experimental approach. Journal of Research in Personality, 40, 529-550. doi: 10.1016/j.jrp.2005.05.003

McNiel, J. M., Lowman, J. C., \& Fleeson, W. (2010). The effect of state extraversion on four types of affect. European Journal of Personality, 24, 18-35. doi: 10.1002/per.738

Michaud, K., Matheson, K., Kelly, O., \& Anisman, H. (2008). Impact of stressors in a natural context on release of cortisol in healthy adult humans: A meta-analysis. Stress: The International Journal on the Biology of Stress, 11, 177-197. doi: $10.1080 / 10253890701727874$

Mischel, W. (1977). The interaction of person and situation. In D. Magnusson \& N. S. Endler (Eds.), Personality at the crossroads: Current issues in interactional 

psychology (pp. 333-352). Hillsdale, NJ: Lawrence Erlbaum Associates, Inc.

Mischel, W. (2009). From Personality and Assessment (1968) to Personality Science, 2009. Journal of Research in Personality, 43, 282-290. doi:

10.1016/j.jrp.2008.12.037

Moskowitz, D. S., \& Côté, S. (1995). Do interpersonal traits predict affect? A comparison of three models. Journal of Personality and Social Psychology, 69, 915-924. doi: $10.1037 / 0022-3514.69 .5 .915$

Muraven, M. R., \& Baumeister, R. F. (2000). Self-regulation and depletion of limited resources: Does self-control resemble a muscle? Psychological Bulletin, 126, 247259. doi: 10.1037.0033-2909.126.2.247

Muraven, M., \& Slessareva, E. (2003). Mechanisms of self-control failure: Motivation and limited resources. Personality and Social Psychology Bulletin, 29, 894-906. doi: $10.1177 / 0146167203029007008$

Muraven, M., Tice, D. M., \& Baumeister, R. F. (1998). Self-control as limited resource: Regulatory depletion patterns. Journal of Personality and Social Psychology, 74, 774-789. doi: 10.1037/0022-3514.74.3.774

Nezlek, J. B. (2011). Multilevel Modeling for Social and Personality Psychology. Thousand Oaks, CA: Sage.

Pavot, W., Diener, E., \& Fujita, F. (1990). Extraversion and happiness. Personality and Individual Differences, 11, 1299-1306. doi: 10.1016/0191-8869(90)90157-M

Pervin, L. A. (1968). Performance and satisfaction as a function of individualenvironment fit. Psychological Bulletin, 69, 56-68. doi: 10.1037/h0025271

Raudenbush, S. W., \& Bryk, A. S. (2002). Hierarchical Linear Models: Applications and 
data analysis methods (2nd ed.). Thousand Oaks: Sage.

Reis, H. T., \& Collins, W. A. (2004). Relationships, human behavior, and psychological science. Current Directions in Psychological Science, 13, 233-237. doi: 10.1111/j.0963-7214.2004.00315.x

Robinson, O. C. (2009). On the social malleability of traits. Variability and consistency in Big 5 trait expression across three interpersonal contexts. Journal of Individual Differences, 30, 201-208. doi: 10.1027/1614-0001.30.4.201

Rogers, C. R. (1961). On becoming a person. Oxford, UK: Houghton Mifflin.

Russell, J. A. (1980). A circumplex model of affect. Journal of Personality and Social Psychology, 39, 1161-1178. doi: 10.1037/h0077714

Russell, J. A., \& Carroll, J. M. (1999). On the bipolarity of positive and negative affect. Psychological Bulletin, 125, 3-30. doi: 10.1037/0033-2909.125.1.3

Rusting, C. L., \& Larsen, R. J. (1997). Extraversion, neuroticism, and susceptibility to positive and negative affect: A test of two theoretical models. Personality and Individual Differences, 22, 607-612. doi: 10.1016/S0191-8869(96)00246-2

Ryan, R. M., \& Deci, E. L. (2004). Autonomy is no illusion: Self-determination theory and the empirical study of authenticity, awareness, and will. In J. Greenberg, S. L. Koole, \& T. Pyszczynski (Eds.), Handbook of experimental existential psychology (pp. 449-479). New York, NY: Guildford Press.

Sandstrom, G. M., \& Dunn, E. W. (2013). Is efficiency overrated? Minimal social interactions lead to belonging and positive affect. Social Psychological and Personality Science. Advanced online publication. doi: $10.1177 / 1948550613502990$ 
Saucier, G. (1994). Mini-markers: A brief version of Goldberg's unipolar Big-Five markers. Journal of Personality Assessment, 63, 506-516. doi: 10.1207/s15327752jpa6303_8

Saucier, G., \& Goldberg, L. R. (2003). The structure of personality attributes. In M. R. Barrick \& A. M. Ryan (Eds.), Personality and work: Reconsidering the role of personality in organizations (pp. 1-29). San Francisco, CA: Jossey-Bass.

Schlegel, R. J., Hicks, J. A., Arndt, J., \& King, L. A. (2009). Thine own self: True selfconcept accessibility and meaning in life. Journal of Personality and Social Psychology, 96, 473-490. doi: 10.1037/a0014060

Sheldon, K. M., Ryan, R. M., Rawsthorne, L. J., \& Ilardi, B. (1997). Trait self and true self: Cross-role variation in the big-five personality traits and its relations with psychological authenticity and subjective well-being. Journal of Personality and Social Psychology, 73, 1380-1393. doi: 10.1037/0022-3514.73.6.1380

Singer, J. D., \& Willett, J. B. (2003). Applied longitudinal data analysis: Modeling change and event occurrence. New York, NY: Oxford University Press.

Sjögren, E., Leanderson, P., Kristenson, M., \& Ernerudh, J. (2006). Interleukin-6 levels in relation to psychosocial factors: Studies on serum, saliva, and in vitro production by blood mononuclear cells. Brain, Behavior, and Immunity, 20, 270278. doi: 10.1016/j.bbi.2005.08.001

Srivastava, S., Angelo, K. M., \& Vallereaux, S. R. (2008). Extraversion and positive affect: Person-environment transactions in daily life. Journal of research in personality, 42, 1613-1618. doi: 10.1016/j.jrp.2008.05.002

Tice, D. M., Baumeister, R. F., Schmueli, D., \& Muraven, M. (2007). Restoring the self: 
Positive affect helps improve self-regulation following ego depletion. Journal of Experimental Social Psychology, 43, 379-384. doi: 10.1016/j.jesp.2006.05.007

Trapnell, P. D., \& Wiggins, J. S. (1990). Extension of the interpersonal adjectives scales to include the Big Five dimensions of personality. Journal of Personality and Social Psychology, 59, 781-790. doi: 10.1037/0022-3514.59.4.781

Turner, R., \& Billings, V. (1991). The social context of self-feeling. In J. Howard \& P. Callero (Eds.), The self-society dynamic: Cognition, emotion, and action (pp. 103122). Cambridge, England: Cambridge University Press.

Tyler, J. M., \& Burns, K. C. (2008). After depletion: The replenishment of the self's regulatory resources. Self and Identity, 7, 305-321. doi: $10.1080 / 15298860701799997$

Vittengl, J. R., \& Holt, C. S. (2000). Getting acquainted: The relationship of selfdisclosure and social attraction to positive affect. Journal of Social and Personal Relationships, 17, 53-66. doi: 10.1177/026507500171003

Vohs, K. D., \& Baumeister, R. F. (Eds.). (2011). Handbook of self-regulation: Research, theory, and applications (2nd ed). New York, NY: The Guildford Press.

Vohs, K. D., Baumeister, R. F., \& Ciarocco, N. J. (2005). Self-regulation and selfpresentation: Regulatory resource depletion impairs impression management and effortful self-presentation depletes regulatory resources. Journal of Personality and Social Psychology, 88, 632-657. doi: 10.1037/0022-3514.88.4.632

Vohs, K. D., \& Faber, R. J. (2007). Spent resources: Self-regulatory resource availability affects impulse buying. Journal of Consumer Research, 33, 537-547. doi: $10.1086 / 510228$ 
Vohs, K. D., \& Heatherton, T. F. (2000). Self-regulatory failure: A resource-depletion approach. Psychological Science, 11, 249-254. doi: 10.1111/1467-9200.00250

Warr, P., Barter, J., \& Brownbridge, G. (1983). On the independence of positive and negative affect. Journal of Personality and Social Psychology, 44, 644-651. doi: $10.1037 /-0022-3514.44 .3 .644$

Watson, D. (1988). Intraindividual and interindividual analyses of positive and negative affect: Their relation to health complaints, perceived stress, and daily activities. Journal of Personality and Social Psychology, 54, 1020-1030. doi: 10.1037/00223514.54.6.1020

Watson, D. \& Clark, L. A. (1997). Extraversion and its positive emotional core. In R. Hogan, J. A. Johnson, \& S. R. Briggs (Eds.), Handbook of personality psychology, (pp. 767-793). San Diego, CA: Academic Press.

Watson, D., Clark, L. A., McIntyre, C. W., \& Hamaker, S. (1992). Affect, personality, and social activity. Journal of Personality and Social Psychology, 63, 1011-1025. doi: $10.1037 / 0022-3514.63 .6 .1011$

Watson, D., Clark, L. A., \& Tellegen, A. (1988). Development and validation of brief measures of positive and negative affect: The PANAS scales. Journal of Personality and Social Psychology, 54, 1063-1070. doi: 10.1037/00223514.54.6.1063

Whelan, D. C., \& Zelenski, J. M. (2007, January). The emotional implications of acting extraverted: Does it depend on disposition? Poster presented at the meeting of the Society for Personality and Social Psychology, Memphis, TN.

Whelan, D. C., \& Zelenski, J. M. (2012a). Experimental evidence that positive moods 
EXTRAVERSION AND COUNTER-DISPOSITIONAL BEHAVIOUR

cause sociability. Social Psychological and Personality Science, 3, 430-437. doi: $10.1177 / 1948550611425194$

Whelan, D. C., \& Zelenski, J. M. (2012b, January). Ideal-self image may explain why counter-dispositional behavior can feel authentic. Poster presented at the annual meeting of the Society for Personality and Social Psychology, San Diego, CA.

Wiggins, J. S. (1979). A psychological taxonomy of trait-descriptive terms: I. The interpersonal domain. Journal of Personality and Social Psychology, 37, 395-412. doi: $10.1037 / 0022-3514.37 .3 .395$

Williams, C. (2006). An investigation of the self-regulatory costs associated with manipulating state extraversion (Undergraduate thesis). Unpublished, Department of Psychology, Carleton University, Ottawa, Canada.

Wilt, J., Noftle, E. E., Fleeson, W., \& Spain, J. S. (2012). The dynamic role of personality states in mediating the relationship between extraversion and positive affect. Journal of Personality, 80, 1205-1236. doi: 10.1111/j.1467-6494.2011.00756.x

Wilt, J., \& Revelle, W. (2009). Extraversion. In M. Leary and R. Hoyle (Eds.), Handbook of individual differences in social behavior (pp. 27-45). New York, NY: Guilford Press.

Wood, A. M., Linley, P. A., Maltby, J., Baliousis, M., \& Joseph, S. (2008). The authentic personality: A theoretical and empirical conceptualization and the development of the authenticity scale. Journal of Counseling Psychology, 55, 385-399. doi: $10.1037 / 0022-0167.55 .3 .385$

Yang, Y., Read, S. J., \& Miller, L. C. (2009). The concept of situations. Social and Personality Psychology Compass, 3, 1018-1037. doi: 10.1111/j.1751- 

9004.2009.00236.x

Zelenski, J. M., \& Larsen, R. J. (1999). Susceptibility to affect: A comparison of three personality taxonomies. Journal of Personality, 67, 761-791. doi: 10.1111/14676494.00072

Zelenski, J. M., \& Larsen, R. J. (2000). The distribution of basic emotions in everyday life: A state and trait perspective from experience sampling data. Journal of Research in Personality, 34, 178-197. doi: 10.1006/jrpe.1999.2275

Zelenski, J. M., Santoro, M. S., \& Whelan, D. C. (2012). Would introverts be better off if they acted more like extraverts? Exploring emotional and cognitive consequences of counter-dispositional behavior. Emotion, 12, 290-303. doi: 10.1037/a0025169

Zelenski, J. M., Sobocko, K., \& Whelan, D. C. (2014). Introversion, solitude, and subjective well-being. In R. J. Coplan \& J. C. Bowker (Eds.), A handbook of solitude: Psychological perspectives on social isolation, social withdrawal, and being alone (pp. 184-201). New York, NY: Wiley-Blackwell.

Zelenski, J. M., Whelan, D. C., Nealis, L. J., Besner, C. M., Santoro, M. S., \& Wynn, J. E. (2013). Personality and affective forecasting: Trait introverts underpredict the hedonic benefits of acting extraverted. Journal of Personality and Social Psychology, 104, 1092-1108. doi: 10.1037/a0032281 


\section{Appendix A: Personality Trait Questionnaire}

Please use this list of common human traits to describe yourself as accurately as possible. Describe yourself as you see yourself at the present time, not as you wish to be in the future. Describe yourself as you are generally or typically, as compared with other persons you know of the same sex and of roughly your same age. Before each trait, please write a number indicating how accurately that trait describes you, using the following rating scale:

Not At

All

1
Somewhat

3
4
Very

Well

\begin{tabular}{|c|c|}
\hline Active & Energetic \\
\hline Assertive & Envious \\
\hline Bashful & Extraverted \\
\hline Bold & _Fretful \\
\hline Careless & _Harsh \\
\hline Cold & - Imaginative \\
\hline Complex & Inefficient \\
\hline Cooperative & Inhibited \\
\hline Creative & Introverted \\
\hline Daring & _Intellectual \\
\hline Deep & _Jealous \\
\hline Disorganized & _Kind \\
\hline Efficier & _Moody \\
\hline
\end{tabular}

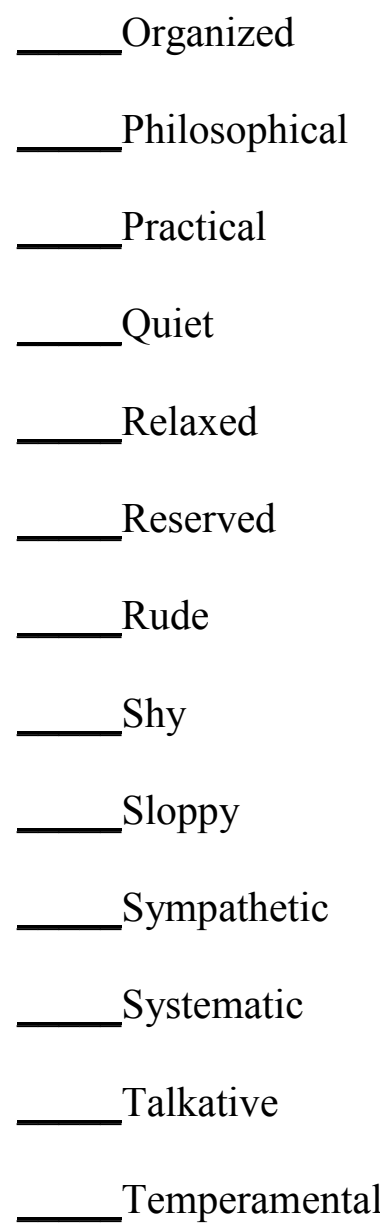

Timid

Touchy

Unadventurous

Uncreative

Unenvious

Unintellectual

Unrestrained

Unsympathetic

Untalkative

Verbal

Vigourous

Warm

ithdrawn 


\section{Appendix B: Affect Questionnaire}

\begin{tabular}{|c|c|c|c|c|c|}
\hline \multicolumn{2}{|c|}{$\begin{array}{c}\text { Very Slightly } \\
\text { or not at all } \\
1\end{array}$} & 3 & der & 5 & 6 \\
\hline & Strong & & & & Irritable \\
\hline & Alert & & & & Upset \\
\hline & Excited & & & & Afraid \\
\hline & Nervous & & & & Sad \\
\hline & Active & & & & Interested \\
\hline & Distressed & & & & Pleased \\
\hline & Happy & & & & Ashamed \\
\hline & Embarrassed & & & & Unhappy \\
\hline & Enthusiastic & & & & Attentive \\
\hline & Hostile & & & & Proud \\
\hline
\end{tabular}

Extremely

or a lot 7 


\section{Appendix C: Affective Forecast Questionnaire}

Take a moment to consider the upcoming activity and your instructions for it. Please try to anticipate your thoughts and feelings during the activity, and indicate this below.

Please indicate your agreement with the following statements:

Strongly

Disagree

1
Neither

Agree nor

Disagree

4

5

6

Strongly

Agree

7

1. I think the interaction will be enjoyable.

2. I will have moments of fun during the interaction.

3. I think I will like the other member.

4. I think the other member will like me.

Please indicate how you think you will feel during the interaction:

Very slightly

or not at all

1
Moderately

4

5
Extremely

or a lot

7
Embarrassed

Happy

Worried

Relaxed

Pleased

Fearful

Enthusiastic
Upset

Excited

Nervous

Distressed

Interested

Self Conscious

Strong 


\section{Appendix D: Behaviour Questionnaire}

Next to each adjective, please write the number that indicates how well the adjective describes the way you acted DURING the activity.

Strongly

Disagree

1

2

3
Neither Agree

nor Disagree

4
Strongly

Agree

7
Rude

Assertive

Trustful

Hardworking

Talkative

Polite

Quiet

Warm

Disorganized 


\section{Appendix E: Authenticity and Effort Questionnaire}

Please answer the following questions regarding how you felt DURING the activity that you just completed.

Strongly

Disagree

1
2

3 
EXTRAVERSION AND COUNTER-DISPOSITIONAL BEHAVIOUR

\section{Appendix F: Opinions About the Activity Questionnaire}

Please indicate your agreement with the following statements:

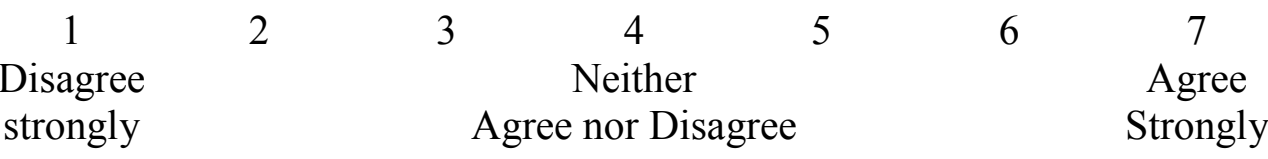

1. The activity was enjoyable.

2. I learned something from the activity.

3. Given the situation, the activity was as much fun as could be expected.

4. I have previously met the other group members.

5. Compared to other experiments I have been in (or expected), this study was reasonably interesting.

6. I had moments of fun during the activity.

7. Time went very slowly for me during the activity. 
Appendix G: Rating of Other Participant Questionnaire - Study 1

Perception of other participants - Please rate participant

Next to each adjective, please write the number that indicates how well the adjective describes participant $\mathrm{X}$ during the activity.

Strongly

Disagree

1

(1)

2

3

Neither Agree

nor Disagree

4
5
Strongly

Agree

7
Energetic Kind Unsympathetic Responsible Shy Cooperative Bold Distrustful Conscientious

I am very confident that the above ratings are an accurate observation of participant X's behaviour during the activity.
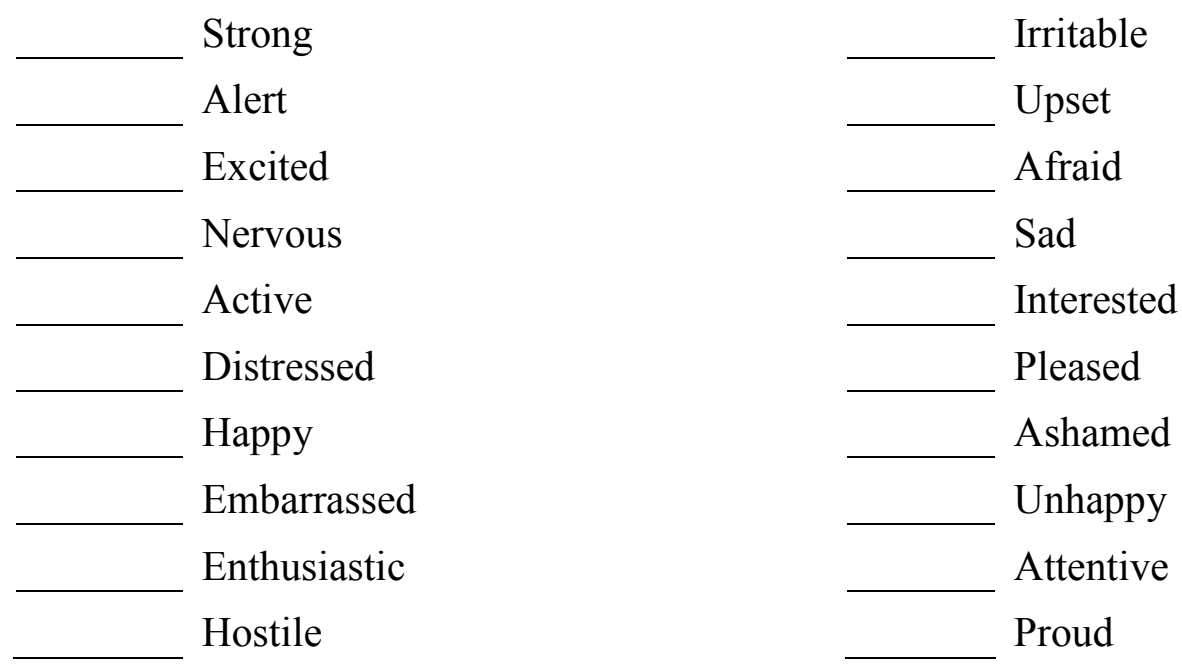

I am very confident that the above ratings are an accurate observation of participant X's feelings during the activity. 


\section{Appendix H: Instructions to Modify Behaviour Questionnaire - Study 1}

\section{Acting extraverted condition}

Specifically, we would like you to be bold, talkative, energetic, active, assertive, and adventurous. During the entire activity, please try to act in those ways.

Please circle the adjective for how you have been instructed to behave.

\begin{tabular}{|c|c|}
\hline Fretful & Extraverted \\
\hline Talkative . & Imaginative \\
\hline Bold & Touchy \\
\hline Careless & Assertive \\
\hline Adventurous & Warm \\
\hline Disorganized & Energetic \\
\hline Active & Jealous \\
\hline
\end{tabular}

\section{Acting introverted condition}

Specifically, we would like you to be reserved, quiet, lethargic, passive, compliant, and unadventurous. During the entire activity, please try to act in those ways.

Please circle the adjective for how you have been instructed to behave.

\begin{tabular}{|c|c|}
\hline Fretful & Introverted \\
\hline Quiet .... & Imaginative \\
\hline Reserved & Touchy \\
\hline Careless & Compliant \\
\hline Unadventurous & Warm \\
\hline Disorganized & Lethargic \\
\hline Passive & Jealous \\
\hline
\end{tabular}


Appendix I: Confederate Behaviour Rating Sheet - Study 2

Next to each adjective, please write the number that indicates how well the adjective describes the way the participant BEHAVED during the interaction.

Strongly

Disagree

1

2

1. Energetic

2. Cooperative

3. Efficient

4. Cold

5. Assertive

6. Insecure

7. Rude

8. Extraverted

9. Careless

10. Talkative

11. Warm

12. Shy

13. Sympathetic

14. Harsh

15. Practical

16. Sloppy

17. Systematic

18. Unenvious

19. Temperamental

20. Complex

21. Relaxed

22. Uncreative

23. Deep

24. Touchy

25. Introverted
Neither Agree

nor Disagree

4

5

26. Kind

27. Withdrawn

28. Bold

29. Disorganized

30. Agreeable

31. Passive

32. Bashful

33. Quiet

34. Nervous

35. Adventurous

36. Considerate

37. Reserved

38. Organized

39. Imaginative

40. Inefficient

41. Unsympathetic

42. Fretful

43. Philosophical

44. Intellectual

45. Unintellectual

46. Moody

47. Jealous

48. Envious

49. Creative

50. Talkative
Strongly

Agree

7 


\section{Appendix J: Confederate Affect Rating Sheet - Study 2}

Next to each adjective, please write the number that indicates how well the adjective describes the way the participant FELT during the interaction.

$\begin{array}{ccccccc}\begin{array}{c}\text { Strongly } \\ \text { Disagree }\end{array} & & \begin{array}{c}\text { Neither Agree } \\ \text { nor Disagree }\end{array} & \begin{array}{c}\text { Strongly } \\ \text { Agree }\end{array} \\ 1 & 2 & 3 & 4 & 5 & 6 & 7\end{array}$

${ }_{\square}$ Strong
Alert
Worried
Excited
Nervous
Active
Tranquil
Distressed
Happy
Embarrassed
Enthusiastic
Hostile
Self-Conscious
Idle

${ }_{\ldots}$ Irritable
Upset
Anxious
Afraid
Sad
Interested
Stimulated
Pleased
Ashamed
Unhappy
Attentive
Proud
Quiet
Fearful


Appendix K: Instructions to Modify Behaviour Questionnaire - Study 2

You will be participating in a work or play activity. This activity will last 25 minutes.

Your activity:

Jigsaw Puzzle

People are different from each other. Please think of a person that fits the following description:

$\mathrm{He} / \mathrm{she}$ is overly social (introduces themself to everyone and instantly become friends), and a chatterbox (non-stop talking). He/she is frequently the individual in a group that is the center of attention due to constantly being busy and loud. He/she would be described as energized by the environment. In a group discussion, he/she blurts out ideas even if interrupting someone else and is highly assertive in influencing group decisions.

During the activity, we would like you to act like the person described above. Please try your best to act in the way described for the entire activity.

This may or may not be the way you normally act - that is ok. Just please try to act in those ways as much as possible during the entire activity.

There is a small prize/reward. To be eligible for this prize, you must prevent the other participant(s) from completing their jigsaw puzzles (i.e., we are asking you to be a mole and to distract the other participant(s) with social chit-chat such that they do not complete their puzzles). All other participants in the experiment must not finish their jigsaw puzzles for you to be eligible for the prize.

Please do not discuss/mention instructions (or that that you have been given instructions) with the other members of the activity! 


\section{Instructions}

You will be participating in a work or play activity. This activity will last 25 minutes.

Your activity:

\section{Jigsaw Puzzle}

People are different from each other. Please think of a person that fits the following description:

$\mathrm{He} / \mathrm{sh}$ is introspective and contemplative (takes their time before speaking). He/she enjoys solitude and being reflective. He/she would be described as energized from within. In a group discussion, he/she is a great listener, reserved but highly observant. Each of the few comments that he/she makes are made deliberately and are relevant to the topic at hand.

During the activity, we would like you to act like the person described above. Please try your best to act in the way described for the entire activity.

This may or may not be the way you normally act - that is ok. Just please try to act in those ways as much as possible during the entire activity.

There is a small prize/reward. To be eligible for this prize, you must complete your jigsaw puzzle.

Please do not discuss/mention instructions (or that that you have been given instructions) with the other members of the activity! 


\section{Instructions}

You will be participating in a work or play activity. This activity will last 25 minutes.

Your activity:

\section{Jigsaw Puzzle}

We are interested in how groups work together on a united goal. We are also interested in how people work independently when other people are in close proximity.

Working with other people in close proximity is a common occurrence in everyday life and we hope to better understand the way in which people interact. Although you may not know the other participants, we believe this situation is realistic of work settings and other group activity formats. Furthermore, open office concepts are common work locations in today's working environments. We hope to better understand the way in which people interact and the individual differences that assist in individuals achieving their objectives.

There is a small prize/reward. To be eligible for this prize, you must complete your jigsaw puzzle.

\section{Please do not discuss instructions with the other members of the activity!}

